DESY 07-117

\title{
Next-to-leading order corrections in exclusive meson production
}

\author{
M. Diehl and W. Kugler \\ Deutsches Elektronen-Synchroton DESY, 22603 Hamburg, Germany
}

\begin{abstract}
We analyze in detail the size of next-to-leading order corrections to hard exclusive meson production within the collinear factorization approach. Corrections to the cross section are found to be huge at small $x_{B}$ and substantial in typical fixed-target kinematics. With the models we take for nucleon helicity-flip distributions, the transverse target polarization asymmetry in vector meson production is strongly affected by radiative corrections, except at large $x_{B}$. Its overall size is very small for $\rho$ production but can be large in the $\omega$ channel.
\end{abstract}




\section{Contents}

1 Introduction $\quad 3$

2 Hard-scattering kernels 4

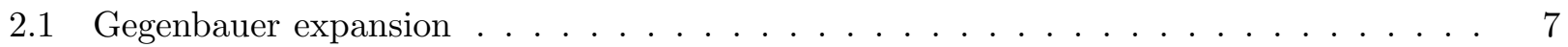

3 Model for the unpolarized GPDs 9

3.1 Nucleon helicity-flip distributions . . . . . . . . . . . . . . . . 10

4 Vector meson production at small $x_{B} \quad 14$

5 Vector meson production at moderate to large $x_{B} \quad 21$

6 Proton helicity flip amplitudes $\quad 28$

\begin{tabular}{ll|l|}
7 & Cross sections and asymmetries & 33 \\
\hline
\end{tabular}

8 Pseudoscalar meson production $\quad 39$

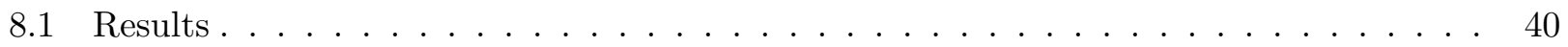

9 Summary $\quad 43$

\begin{tabular}{ll} 
A Polylogarithms & 44 \\
\hline
\end{tabular}

B Hard-scattering kernels for higher Gegenbauer moments 44

$\begin{array}{lll}\text { C Evolution kernels } & 46\end{array}$ 


\section{Introduction}

Generalized parton distributions (GPDs) have developed into a versatile tool to quantify important aspects of hadron structure in QCD. In particular they contain unique information on the transverse spatial distribution of partons [1] and on spin-orbit effects and orbital angular momentum inside the nucleon [2, 3]. Deeply virtual Compton scattering is widely recognized as the process providing the theoretically cleanest access to GPDs, with a wealth of observables calculable in the large $Q^{2}$ limit [4] and with the calculation of the hard-scattering subprocess now pushed to next-to-next-toleading order (NNLO) accuracy in $\alpha_{s}$ [5. A quantitative theoretical description of exclusive meson production remains a challenge. It would offer the possibility to obtain important complementary information, difficult to obtain from Compton scattering alone. Perhaps most importantly, vector meson production is directly sensitive to gluon distributions, which in the Compton process are $\alpha_{s}$ suppressed relative to quark distributions and only accessible through scaling violation (just as in the well-known case of inclusive deep inelastic scattering). Given in addition the large number of channels that can be studied and the wealth of high-quality data in a wide range of kinematics from collider to fixed-target energies [6, 7], it should be worthwhile to try and push the theory description of exclusive meson production as far as possible.

In this work we study the exclusive production of light mesons at large photon virtuality $Q^{2}$ within the framework of collinear factorization [8]. In Bjorken kinematics, the process amplitude can be approximated by the convolution of hard-scattering kernels with generalized parton distributions and the quark-antiquark distribution amplitude of the produced meson. The hard-scattering kernels have been calculated to $O\left(\alpha_{s}^{2}\right)$, i.e. to next-to-leading order (NLO) accuracy [9, 10, 11]. The aim of the present paper is to investigate in some detail the size of the NLO corrections compared with the leading-order (LO) results, on which phenomenological studies have so far relied.

The collinear factorization approach provides an approximation of the leading helicity amplitudes for meson production in the Bjorken limit, up to relative corrections of order $1 / Q^{2}$. These power corrections cannot be calculated systematically (and in fact the derivation [8] of the factorization theorem suggests that these corrections do not all factorize into hard-scattering kernels and nonperturbative quantities pertaining to either the nucleon or the produced meson). One particular source of power corrections can however readily be identified, namely the effect of the transverse momentum of partons entering the hard-scattering subprocess, which in the collinear approximation is neglected in the calculation of the hard-scattering kernel. A number of approaches include these $k_{T}$ effects, in particular the studies in [12, 13] based on the modified hard-scattering formalism of Sterman et al. [14], and calculations like [15] which are based on the color dipole formulation. In the work by Martin, Ryskin and Teubner [16], parton-hadron duality is used to model the meson formation and thus the transverse momentum of the hadronizing quarks is included in the calculation, whereas the transverse momentum of gluons in the proton is treated within high-energy $k_{T}$ factorization. The studies just quoted agree in that transverse momentum effects result in substantial power corrections to the collinear approximation for $Q^{2}$ up to several $\mathrm{GeV}^{2}$. Unfortunately, the calculation of full NLO corrections in $\alpha_{s}$ remains not only a practical but also a conceptual challenge in all of these approaches, so that the perturbative stability of their results cannot be investigated at present. (The approach of Sterman et al. takes partial account of radiative corrections, resumming a certain class of them into Sudakov form factors.)

A consistent simultaneous treatment of radiative and power corrections being out of reach at this time, a possible strategy is to study the NLO corrections in the collinear approximation and in particular to identify kinematical regions where these corrections are moderate or small. There one can then use with greater confidence formulations incorporating power corrections. In this spirit the present investigation should be understood. We will study both the cross section for meson production 
from an unpolarized target and the transverse target polarization asymmetry. This asymmetry is one of the few observables sensitive to the nucleon helicity-flip distributions (in particular for gluons) and hence to the spin-orbit and orbital angular momentum effects mentioned above. We will in particular see whether corrections tend to cancel in this polarization asymmetry, as is often assumed.

In the bulk of this paper we concentrate on the production of vector mesons. In Sect. 2 we set up our notation and recall important properties of the hard-scattering kernels at NLO, as well as giving a one-variable representation of these kernels after Gegenbauer expansion of the meson distribution amplitude. In Sect. 3 we specify the model of the generalized parton distributions $H$ and $E$ we use for our numerical studies. The size of radiative corrections involving convolutions with distributions $H$ is then studied in Sects. 4 and 5 for small and large $x_{B}$, respectively, and the convolutions involving distributions $E$ are quantified in Sect.6. In Sect. 7 we then look at the NLO corrections at the level of the observable cross section and polarization asymmetry. A brief study of exclusive pion production in Sect. 8 complements our work, and in Sect. 9 we summarize our main findings. A number of more lengthy formulae is collected in appendices.

\section{Hard-scattering kernels}

In the main part of this paper we are concerned with exclusive production of a vector meson

$$
\gamma^{*}(q)+p(p) \rightarrow V\left(q^{\prime}\right)+p\left(p^{\prime}\right)
$$

in the limit of large $Q^{2}=-q^{2}$ at fixed Bjorken variable $x_{B}=Q^{2} /(2 p \cdot q)$ and fixed $t=\left(p-p^{\prime}\right)^{2}$. To leading order in $1 / Q$, the amplitude for longitudinal polarization of photon and meson can be written as

$$
\begin{aligned}
& \mathcal{M}=\frac{2 \pi \sqrt{4 \pi \alpha}}{\xi Q N_{c}} Q_{V} f_{V} \int_{0}^{1} d z \phi_{V}(z) \int_{-1}^{1} d x\left\{T_{g}(z, x, \xi) F^{g}(x, \xi, t)\right. \\
& +\frac{1}{n_{f}}\left[T_{a}(\bar{z}, x, \xi)-T_{a}(z,-x, \xi)\right] F^{S}(x, \xi, t)+T_{b}(z, x, \xi) F^{S}(x, \xi, t) \\
& +e_{V}^{(3)}\left[T_{a}(\bar{z}, x, \xi)-T_{a}(z,-x, \xi)\right]\left[F^{u(+)}(x, \xi, t)-F^{d(+)}(x, \xi, t)\right] \\
& \left.+e_{V}^{(8)}\left[T_{a}(\bar{z}, x, \xi)-T_{a}(z,-x, \xi)\right]\left[F^{u(+)}(x, \xi, t)+F^{d(+)}(x, \xi, t)-2 F^{s(+)}(x, \xi, t)\right]\right\}
\end{aligned}
$$

with $\bar{z}=1-z, N_{c}=3$, and the electromagnetic fine structure constant $\alpha$. Throughout this paper we work with $n_{f}=3$ active quark flavors. The proton matrix elements $F$ are parameterized by generalized parton distributions,

$$
F^{q, g}(x, \xi, t)=\frac{1}{\left(p+p^{\prime}\right) \cdot n}\left[H^{q, g}(x, \xi, t) \bar{u}\left(p^{\prime}\right) \not h u(p)+E^{q, g}(x, \xi, t) \bar{u}\left(p^{\prime}\right) \frac{i \sigma^{\alpha \beta} n_{\alpha}\left(p^{\prime}-p\right)_{\beta}}{2 m_{p}} u(p)\right]
$$

for quarks and gluons, where we use the conventions of [17]. Here $n$ is a light-like auxiliary vector, $\xi=x_{B} /\left(2-x_{B}\right)$ is the skewness variable, and $m_{p}$ denotes the nucleon mass. We have further introduced the combination

$$
F^{q(+)}(x, \xi, t)=F^{q}(x, \xi, t)-F^{q}(-x, \xi, t)
$$

with positive charge conjugation parity. In (2) we have arranged the terms containing quark distributions into the flavor singlet

$$
F^{S}=F^{u(+)}+F^{d(+)}+F^{s(+)}
$$



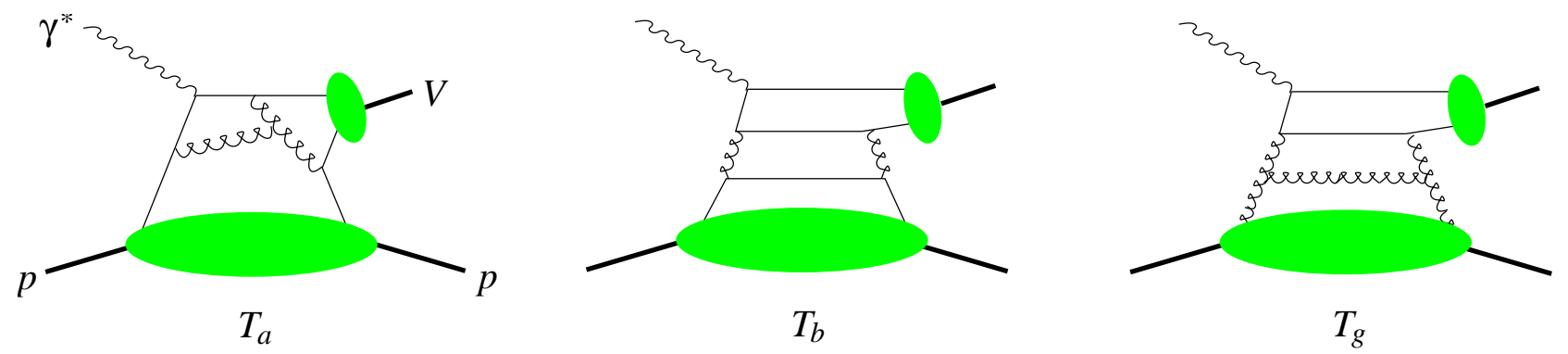

Figure 1: Example graphs for the hard-scattering kernels $T_{a}, T_{b}$ and $T_{g}$ at order $\alpha_{s}^{2}$.

and the flavor triplet and octet combinations, $F^{u(+)}-F^{d(+)}$ and $F^{u(+)}+F^{d(+)}-2 F^{s(+)}$. The factors

$$
Q_{\rho}=\frac{1}{\sqrt{2}}, \quad Q_{\omega}=\frac{1}{3 \sqrt{2}}, \quad Q_{\phi}=-\frac{1}{3}
$$

and

$$
e_{\rho}^{(3)}=e_{\rho}^{(8)}=e_{\omega}^{(8)}=\frac{1}{6}, \quad e_{\omega}^{(3)}=\frac{3}{2}, \quad e_{\phi}^{(3)}=0, \quad e_{\phi}^{(8)}=-\frac{1}{3}
$$

correspond to a respective flavor content

$$
\frac{1}{\sqrt{2}}(|u \bar{u}\rangle-|d \bar{d}\rangle), \quad \frac{1}{\sqrt{2}}(|u \bar{u}\rangle+|d \bar{d}\rangle), \quad|s \bar{s}\rangle
$$

of the $\rho, \omega$ and $\phi$. The meson distribution amplitudes $\phi_{V}(z)$ are normalized as $\int_{0}^{1} d z \phi_{V}(z)=1$, and the decay constants have the values $f_{\rho}=209 \mathrm{MeV}, f_{\omega}=187 \mathrm{MeV}, f_{\phi}=221 \mathrm{MeV}$ [18]. We finally have hard-scattering kernels in (2), where $T_{g}$ goes with gluon and $T_{a}, T_{b}$ go with quark distributions in the proton. In the graphs for $T_{a}$ quark lines connect the proton and meson side, whereas in the graphs for $T_{b}$ the proton and meson side are only connected by gluon lines. $T_{b}$ thus starts at order $\alpha_{s}^{2}$ and only goes with the quark singlet distribution $F^{S}$. Example graphs for the three kernels at NLO are shown in Fig. 1. We will refer to $T_{g}, T_{a}, T_{b}$ as the gluon, the quark non-singlet, and the pure quark singlet kernel, respectively.

For better legibility we have not displayed the dependence on the renormalization and factorization scales in (2). The renormalization scale $\mu_{R}$ appears as argument of $\alpha_{s}$ and through explicit logarithms in the hard-scattering kernels $T$. The kernels further contain logarithms of the respective factorization scales $\mu_{D A}$ and $\mu_{G P D}$ for the meson distribution amplitude and the generalized parton distributions. The NLO kernels in [10, 11] are given for a common factorization scale $\mu_{F}=\mu_{D A}=\mu_{G P D}$. We can restore the individual logarithms of $\mu_{D A}$ and $\mu_{G P D}$ from the requirement that within the calculated precision the process amplitude (2) must be independent of these scales. As an example consider the term

$$
\begin{aligned}
& \frac{\mathrm{d}}{\mathrm{d} \ln \mu_{D A}^{2}} \int_{0}^{1} \mathrm{~d} z \phi_{V}\left(z ; \mu_{D A}\right) T_{a}\left(\bar{z}, x, \xi ; \alpha_{s}\left(\mu_{R}\right), \mu_{R}, \mu_{G P D}, \mu_{D A}, Q\right) \\
& \quad=\int_{0}^{1} d z\left[\frac{\mathrm{d}}{\mathrm{d} \ln \mu_{D A}^{2}} \phi_{V}\left(z ; \mu_{D A}\right)\right] T_{a}\left(\bar{z}, x, \xi ; \alpha_{s}\left(\mu_{R}\right), \mu_{R}, \mu_{G P D}, \mu_{D A}, Q\right) \\
& \quad+\int_{0}^{1} d z \phi_{V}\left(z ; \mu_{D A}\right)\left[\frac{\mathrm{d}}{\mathrm{d} \ln \mu_{D A}^{2}} T_{a}\left(\bar{z}, x, \xi ; \alpha_{s}\left(\mu_{R}\right), \mu_{R}, \mu_{G P D}, \mu_{D A}, Q\right)\right],
\end{aligned}
$$

where the scale dependence of $\phi_{V}\left(z ; \mu_{D A}\right)$ is given by the ERBL evolution equation [19]. At leading order this gives a term $\mathrm{d} / \mathrm{d}\left(\ln \mu_{D A}^{2}\right) \phi_{V}\left(z ; \mu_{D A}\right)$ of order $\alpha_{s}$, whose convolution with the $O\left(\alpha_{s}\right)$ 
part of $T_{a}$ must cancel against the contribution from explicit logarithms of $\mu_{D A}$ in the $O\left(\alpha_{s}^{2}\right)$ part of $T_{a}$. An analogous argument holds for the dependence on $\mu_{G P D}$, with the complication that the gluon and quark singlet distributions mix under evolution. More precisely, the convolution of $\mathrm{d} / \mathrm{d}\left(\ln \mu_{G P D}^{2}\right) F^{S}\left(x, \xi, t ; \mu_{G P D}\right)$ with the $O\left(\alpha_{s}\right)$ part of $T_{a}$ cancels at $O\left(\alpha_{s}^{2}\right)$ against the contributions from logarithms of $\mu_{G P D}$ in $T_{a}$ and in $T_{g}$. Likewise, the convolution of $\mathrm{d} / \mathrm{d}\left(\ln \mu_{G P D}^{2}\right) F^{g}\left(x, \xi, t ; \mu_{G P D}\right)$ with the Born term of $T_{g}$ cancels at $O\left(\alpha_{s}^{2}\right)$ against the contributions from logarithms of $\mu_{G P D}$ in $T_{g}$ and in the pure singlet kernel $T_{b}$. We have explicitly checked that the scale dependence of the hardscattering kernels given in [11] cancels in the process amplitude (2) as just described, using the LO evolution equations for GPDs given in App. C.

Separating the $\mu_{D A}$ and $\mu_{G P D}$ dependence, we can write the kernels as

$$
\begin{aligned}
& T_{g}(z, x, \xi)=-\alpha_{s} \frac{\xi}{(\xi-x-i \epsilon)(\xi+x-i \epsilon)} \frac{1}{z \bar{z}}\left[1+\frac{\alpha_{s}}{4 \pi} \mathcal{I}_{g}\left(z, \frac{\xi-x}{2 \xi}\right)\right], \\
& T_{b}(z, x, \xi)=C_{F} \frac{\alpha_{s}^{2}}{8 \pi} \frac{1}{z \bar{z}} \mathcal{I}_{b}\left(z, \frac{\xi-x}{2 \xi}\right), \\
& T_{a}(\bar{z}, x, \xi)=-C_{F} \alpha_{s} \frac{\xi}{\xi-x-i \epsilon} \frac{1}{\bar{z}}\left[1+\frac{\alpha_{s}}{4 \pi} \mathcal{I}_{a}\left(\bar{z}, \frac{\xi-x}{2 \xi}\right)\right]
\end{aligned}
$$

with

$$
\begin{aligned}
\mathcal{I}_{g}(z, y)= & {\left[2 C_{A}\left(\frac{\bar{y}}{y}+\frac{y}{\bar{y}}\right)(y \ln y+\bar{y} \ln \bar{y})-C_{F}\left(\frac{y}{\bar{y}} \ln y+\frac{\bar{y}}{y} \ln \bar{y}\right)\right] \ln \frac{Q^{2}}{\mu_{G P D}^{2}} } \\
& +\beta_{0} \ln \frac{\mu_{R}^{2}}{\mu_{G P D}^{2}}+C_{F}(3+2 z \ln \bar{z}+2 \bar{z} \ln z) \ln \frac{Q^{2}}{\mu_{D A}^{2}}+\mathcal{K}_{g}(z, y), \\
\mathcal{I}_{b}(z, y)= & 2(\bar{y}-y)\left(\frac{\ln y}{\bar{y}}+\frac{\ln \bar{y}}{y}\right) \ln \frac{Q^{2}}{\mu_{G P D}^{2}}+\mathcal{K}_{b}(z, y)
\end{aligned}
$$

and

$$
\begin{aligned}
\mathcal{I}_{a}(v, u)= & \beta_{0}\left(\frac{5}{3}-\ln (v u)-\ln \frac{Q^{2}}{\mu_{R}^{2}}\right)+C_{F}(3+2 \ln u) \ln \frac{Q^{2}}{\mu_{G P D}^{2}}+C_{F}(3+2 \ln v) \ln \frac{Q^{2}}{\mu_{D A}^{2}} \\
& +\mathcal{K}_{a}(v, u),
\end{aligned}
$$

where $\bar{y}=1-y$ and we use the standard notation

$$
C_{F}=\frac{N_{c}^{2}-1}{2 N_{c}}, \quad C_{A}=N_{c}, \quad \beta_{0}=\frac{11}{3} N_{c}-\frac{2}{3} n_{f} .
$$

The functions $\mathcal{K}_{g}, \mathcal{K}_{b}$ and $\mathcal{K}_{a}$ are independent of $Q^{2}$ and the renormalization and factorization scales. They contain factors $C_{F}$ or $C_{A}$ but not $\beta_{0}$. Their expressions can be found in [11, taking into account that the kernels $T_{g}$ and $T_{b}$ here are denoted by $T_{g}$ and $T_{(+)}$there, and that

$$
\left.T(v, u)\right|_{[11]}=\frac{C_{F} \alpha_{s}}{4 v u}\left[1+\frac{\alpha_{s}}{4 \pi} \mathcal{I}_{a}(v, u)\right]_{\text {here }},\left.\quad y\right|_{[11]}=-\left.y\right|_{\text {here }} .
$$

Note that the pure singlet kernel $T_{b}$ does not contain logarithms of $\mu_{D A}$ and $\mu_{R}$ at $O\left(\alpha_{s}^{2}\right)$, since there is no Born level contribution against which they could cancel in the scale dependence of the process amplitude. There is however a logarithm of $\mu_{G P D}$, since the corresponding derivative of the Born level 
convolution of $T_{g}$ with $F^{g}$ contains a term going with the quark singlet distribution $F^{S}$, as already mentioned after (9).

The kernels in (10) have singularities for real-valued arguments. One readily finds that $x / \xi=$ $(\hat{s}-\hat{u}) / Q^{2}$, where $\hat{s}$ and $\hat{u}$ are the Mandelstam variables for the parton-level subprocess $\gamma^{*} q \rightarrow(q \bar{q}) q$ or $\gamma^{*} g \rightarrow(q \bar{q}) g$. The prescriptions $\hat{s}+i \epsilon$ for the $\hat{s}$-channel and $\hat{u}+i \epsilon$ for the $\hat{u}$-channel singularities thus instruct us to take $x+i \epsilon$ for $x>0$ and $x-i \epsilon$ for $x<0$. Correspondingly, the second argument $(\xi-x) /(2 \xi)$ of $\mathcal{I}_{g}, \mathcal{I}_{b}$ and $\mathcal{I}_{a}$ must be taken with $-i \epsilon$ for $x>0$ and $+i \epsilon$ for $x<0$. In $T_{a}(z,-x, \xi)$ the second argument of $\mathcal{I}_{a}$ is $(\xi+x) /(2 \xi)$, which has to be taken with $-i \epsilon$ for $x<0$ and $+i \epsilon$ for $x>0$. We remark that, as it is written, the $i \epsilon$ prescription in [11] for the gluon and the pure singlet kernel is correct for $x>0$ but incorrect for $x<0$. Likewise, the prescription given in [10, 11] for the quark non-singlet kernel is correct for $x>0$ if the corresponding argument is $(\xi-x) /(2 \xi)$ and for $x<0$ if the argument is $(\xi+x) /(2 \xi)$, but incorrect in the other cases 1

\subsection{Gegenbauer expansion}

Let us expand the meson distribution amplitude on Gegenbauer polynomials,

$$
\phi_{V}(z ; \mu)=6 z(1-z) \sum_{n=0}^{\infty} a_{n}(\mu) C_{n}^{3 / 2}(2 z-1),
$$

where $a_{0}=1$ according to the normalization condition $\int_{0}^{1} d z \phi_{V}(z)=1$. To leading order, the Gegenbauer coefficients evolve as

$$
a_{n}(\mu)=a_{n}\left(\mu_{0}\right)\left(\frac{\alpha_{s}(\mu)}{\alpha_{s}\left(\mu_{0}\right)}\right)^{\gamma_{n} / \beta_{0}}
$$

with anomalous dimensions

$$
\gamma_{0}=0, \quad \gamma_{2}=\frac{25}{6} C_{F}, \quad \gamma_{4}=\frac{91}{15} C_{F}
$$

where $\alpha_{s}(\mu)$ is the running coupling at one-loop accuracy. One has $\gamma_{n} \approx 4 C_{F} \ln (n+1)$ within at most $6 \%$ for all $n$. For $V=\rho, \omega, \phi$ only coefficients $a_{n}$ with even $n$ are nonzero due to charge conjugation invariance, and in all subsequent expressions of this paper we consider $n$ to be even. Calculations of the distribution amplitudes in models or on the lattice typically give values for the first or the first two nonvanishing moments, see e.g. [20, 21, 22], so that a truncated version of the expansion (15)) is very often used in phenomenological studies. Convolution with individual terms in (15) also allows us to reduce the hard-scattering kernels for meson production to functions of a single longitudinal variable. More precisely, we can rewrite the process amplitude (2) as

$$
\mathcal{M}=\frac{2 \pi \sqrt{4 \pi \alpha}}{\xi Q N_{c}} Q_{V} f_{V} \sum_{n=0}^{\infty} a_{n}\left[\mathcal{F}_{n}^{g}+\mathcal{F}_{n}^{S(a)}+\mathcal{F}_{n}^{S(b)}+e_{V}^{(3)} \mathcal{F}_{n}^{(3)}+e_{V}^{(8)} \mathcal{F}_{n}^{(8)}\right]
$$

with convolutions in $x$

$$
\begin{array}{rlr}
\mathcal{F}_{n}^{g} & =\int_{-1}^{1} d x T_{g, n}(x, \xi) F^{g}(x, \xi, t), & \mathcal{F}_{n}^{S(b)}=\int_{-1}^{1} d x T_{b, n}(x, \xi) F^{S}(x, \xi, t), \\
\mathcal{F}_{n}^{S(a)} & =\int_{-1}^{1} d x\left[T_{a, n}(x, \xi)-T_{a, n}(-x, \xi)\right] \frac{1}{n_{f}} F^{S}(x, \xi, t), \\
\mathcal{F}_{n}^{(3)} & =\int_{-1}^{1} d x\left[T_{a, n}(x, \xi)-T_{a, n}(-x, \xi)\right]\left[F^{u(+)}(x, \xi, t)-F^{d(+)}(x, \xi, t)\right],
\end{array}
$$

\footnotetext{
${ }^{1}$ We thank Dima Ivanov for discussions on this point. The numerical results in 11 were obtained with the correct prescription.
} 


$$
\mathcal{F}_{n}^{(8)}=\int_{-1}^{1} d x\left[T_{a, n}(x, \xi)-T_{a, n}(-x, \xi)\right]\left[F^{u(+)}(x, \xi, t)+F^{d(+)}(x, \xi, t)-2 F^{s(+)}(x, \xi, t)\right]
$$

which depend on $\xi$ and $t$, and logarithmically on $Q^{2}$ and on the factorization and renormalization scales. At order $\alpha_{s}^{2}$ the dependence on $\mu_{R}$ and on $\mu_{D A}$ cancels in each separate convolution, while the dependence on $\mu_{G P D}$ cancels in $\mathcal{F}_{n}^{(3)}$ and $\mathcal{F}_{n}^{(8)}$ and in the sum $\mathcal{F}_{n}^{g}+\mathcal{F}_{n}^{S(a)}+\mathcal{F}_{n}^{S(b)}$ as discussed after (9). In analogy to (19) we define convolutions $\mathcal{H}$ and $\mathcal{E}$ for the individual distributions $H$ and $E$ in (3). The kernels $T_{g, n}, T_{a, n}, T_{b, n}$ are obtained from $T_{g}, T_{a}, T_{b}$ by multiplying with $6 z(1-z) C_{n}^{3 / 2}(2 z-1)$ and integrating over $z$. For $n=0$ we find

$$
\begin{aligned}
& T_{g, n}(x, \xi)=-3 \alpha_{s} \frac{2 \xi}{(\xi-x-i \epsilon)(\xi+x-i \epsilon)}\left[1+\frac{\alpha_{s}}{4 \pi} t_{g, n}\left(\frac{\xi-x}{2 \xi}\right)\right], \\
& T_{b, n}(x, \xi)=3 C_{F} \frac{\alpha_{s}^{2}}{4 \pi} t_{b, n}\left(\frac{\xi-x}{2 \xi}\right), \\
& T_{a, n}(x, \xi)=-3 C_{F} \alpha_{s} \frac{\xi}{\xi-x-i \epsilon}\left[1+\frac{\alpha_{s}}{4 \pi} t_{a, n}\left(\frac{\xi-x}{2 \xi}\right)\right]
\end{aligned}
$$

with

$$
\begin{aligned}
t_{g, 0}(y)= & {\left[2 C_{A}\left(y^{2}+\bar{y}^{2}\right)-C_{F} y\right] \frac{\ln y}{\bar{y}} \ln \frac{Q^{2}}{\mu_{G P D}^{2}}+\frac{\beta_{0}}{2} \ln \frac{\mu_{R}^{2}}{\mu_{G P D}^{2}} } \\
& +C_{F}\left[-\frac{5}{2}+\left(\frac{1}{\bar{y}}+1-4 y\right) \ln y-\frac{y}{2} \frac{\ln ^{2} y}{\bar{y}}\right. \\
& \left.\quad-2(\bar{y}-y) \operatorname{Li}_{2} \bar{y}-4 y \bar{y}\left(3 \operatorname{Li}_{3} \bar{y}-\ln y \operatorname{Li}_{2} y-\frac{\pi^{2}}{6} \ln y\right)\right] \\
& +C_{A}\left[-\left(\frac{6}{\bar{y}}-8 y\right) \ln y+\left(\frac{1}{\bar{y}}-2 y\right) \ln ^{2} y+2(\bar{y}-y) \operatorname{Li}_{2} \bar{y}\right]+\{y \rightarrow \bar{y}\} \\
t_{b, 0}(y)= & 2(\bar{y}-y) \frac{\ln y}{\bar{y}}\left[\ln \frac{Q^{2}}{\mu_{G P D}^{2}}-3\right]+(\bar{y}-y) \frac{\ln ^{2} y}{\bar{y}}+4 \operatorname{Li}_{2} \bar{y}-\{y \rightarrow \bar{y}\} \\
t_{a, 0}(y)= & \beta_{0}\left[\frac{19}{6}-\ln y-\ln \frac{Q^{2}}{\mu_{R}^{2}}\right]+C_{F}\left[(3+2 \ln y) \ln \frac{Q^{2}}{\mu_{G P D}^{2}}-\frac{77}{6}-\left(\frac{1}{\bar{y}}-3\right) \ln y+\ln ^{2} y\right] \\
& +\left(2 C_{F}-C_{A}\right)\left\{-\frac{1}{3}-4(2-3 y) \ln \bar{y}+2(1-6 y) \ln y+4(1-3 y)\left(\operatorname{Li}_{2} y-\operatorname{Li}_{2} \bar{y}\right)\right. \\
& \left.\quad+2(1-6 y \bar{y})\left[3\left(\operatorname{Li}_{3} \bar{y}+\operatorname{Li}_{3} y\right)-\ln y \operatorname{Li}_{2} y-\ln \bar{y} \operatorname{Li}_{2} \bar{y}-\frac{\pi^{2}}{6}(\ln y+\ln \bar{y})\right]\right\} .
\end{aligned}
$$

The corresponding kernels for $n=2$ and $n=4$ are given in App. B. The $i \epsilon$ prescription to be used in (20) is the same as specified at the end of the previous subsection. This implies that in $t_{g, n}(y)$, $t_{b, n}(y), t_{a, n}(y)$ and $t_{a, n}(\bar{y})$ one has to take $\ln (y-i \epsilon), \operatorname{Li}_{2}(\bar{y}+i \epsilon)$ and $\operatorname{Li}_{3}(\bar{y}+i \epsilon)$ for $y<0$. For the gluon and pure singlet kernel, which dominate in process amplitudes at small $\xi$, we have in particular

$$
\begin{aligned}
\frac{1}{\pi} \operatorname{Im} t_{g, 0}(y)= & -\left[2 C_{A}\left(y^{2}+\bar{y}^{2}\right)-C_{F} y\right] \frac{1}{\bar{y}} \ln \frac{Q^{2}}{\mu_{G P D}^{2}} \\
& -C_{F}\left[1-4 y+\frac{1-y \ln (-y)}{\bar{y}}+2(\bar{y}-y) \ln \bar{y}+2 y \bar{y}\left(\ln ^{2} \bar{y}+2 \operatorname{Li}_{2} y+\frac{\pi^{2}}{3}\right)\right] \\
& +2 C_{A}\left[\frac{3}{\bar{y}}-4 y-\left(\frac{1}{\bar{y}}-2 y\right) \ln (-y)+(\bar{y}-y) \ln \bar{y}\right],
\end{aligned}
$$




$$
\frac{1}{\pi} \operatorname{Im} t_{b, 0}(y)=2 \frac{\bar{y}-y}{\bar{y}}\left[3-\ln (-y)-\ln \frac{Q^{2}}{\mu_{G P D}^{2}}\right]+4 \ln \bar{y}
$$

in the region $y<0$. In the limit $y \rightarrow 0$ all three expressions in (21) contain singular terms proportional to $\ln y$ and $\ln ^{2} y$. For the convolution (19) we should however consider $(y \bar{y})^{-1} t_{g, n}(y), y^{-1} t_{a, n}(y)$ and $\bar{y}^{-1} t_{a, n}(\bar{y})$ according to (20). With the appropriate $i \epsilon$ prescription, these kernels contain terms which for $y \rightarrow 0$ go like $(y-i \epsilon)^{-1} \ln ^{m}(y-i \epsilon)$, where $m=0,1,2$.

\section{$3 \quad$ Model for the unpolarized GPDs}

It is difficult to study the impact of NLO corrections at the level of the hard-scattering kernels given in the previous subsection, especially since they are not smooth functions but distributions with singularities at $y=0$. We will therefore use model GPDs to investigate the radiative corrections at the level of the convolution integrals (19). The aim of this work is not a systematic improvement of existing models, nor a detailed exploration of model uncertainties on observables in exclusive meson production. We do however require that the models we use are consistent with known theoretical requirements and basic phenomenological constraints.

For $H^{q}$ and $H^{g}$ we adopt the widely used ansatz of [26, 27] based on double distributions, where a $\xi$ dependence is generated according to

$$
\begin{aligned}
H^{q(+)}(x, \xi, t) & =\int_{-1}^{1} d \beta \int_{-1+|\beta|}^{1-|\beta|} d \alpha \delta(x-\beta-\xi \alpha) h^{(2)}(\beta, \alpha) H^{q(+)}(\beta, 0, t), \\
H^{g}(x, \xi, t) & =\int_{-1}^{1} d \beta \int_{-1+|\beta|}^{1-|\beta|} d \alpha \delta(x-\beta-\xi \alpha) h^{(2)}(\beta, \alpha) H^{g}(\beta, 0, t)
\end{aligned}
$$

with

$$
h^{(b)}(\beta, \alpha)=\frac{\Gamma(2 b+2)}{2^{2 b+1} \Gamma^{2}(b+1)} \frac{\left[(1-|\beta|)^{2}-\alpha^{2}\right]^{b}}{(1-|\beta|)^{2 b+1}} .
$$

The distributions at zero skewness are taken as

$$
\begin{aligned}
H^{q(+)}(x, 0, t) & =q_{v}(x) \exp \left[t f_{q_{v}}(x)\right]+2 \bar{q}(x) \exp \left[t f_{\bar{q}}(x)\right], \\
H^{g}(x, 0, t) & =x g(x) \exp \left[t f_{g}(x)\right]
\end{aligned}
$$

for $x>0$, with the values for $x<0$ following from the symmetry properties of the distributions. Here $q_{v}(x)=q(x)-\bar{q}(x), \bar{q}(x)$ and $g(x)$ are the usual unpolarized densities for valence quarks, antiquarks and gluons, for which we take the CTEQ6M parameterization [29]. This parameterization has an identical strange and antistrange sea, so that $s_{v}(x)=0$. The ansatz (23) is taken at a starting scale $\mu_{0}$ and then evolved with the LO evolution equations given in App. C] For the studies in Sects. 4 and 5 we take $\mu_{0}=1.3 \mathrm{GeV}$, which is the starting scale of evolution for the CTEQ6M densities. In Sects. 6] and 7 we will instead take $\mu_{0}=2 \mathrm{GeV}$, since this will allow us to use the results for the $t$ dependence of valence distributions obtained in [28].

For the $t$ dependence in the ansatz (23) we follow the modeling strategy of [27] and take an exponential behavior in $t$ with an $x$ dependent slope. For valence quarks we take the slope functions

$$
f_{q_{v}}(x)=\alpha_{v}^{\prime}(1-x)^{3} \ln \frac{1}{x}+B_{q_{v}}(1-x)^{3}+A_{q_{v}} x(1-x)^{2}
$$

with parameters $\alpha_{v}^{\prime}=0.9 \mathrm{GeV}^{-2}$ and 


$$
\begin{array}{ll}
A_{u_{v}}=1.26 \mathrm{GeV}^{-2}, & B_{u_{v}}=0.59 \mathrm{GeV}^{-2}, \\
A_{d_{v}}=3.82 \mathrm{GeV}^{-2}, & B_{d_{v}}=0.32 \mathrm{GeV}^{-2},
\end{array}
$$

from [28]. We recall the sum rule

$$
F_{1}^{q}(t)=\int_{-1}^{1} d x H^{q}(x, 0, t)=\int_{0}^{1} d x q_{v}(x) \exp \left[t f_{q_{v}}(x)\right]
$$

from which one obtains the electromagnetic Dirac form factors of proton and neutron by appropriate quark flavor combinations. Together with the CTEQ6M distributions at $\mu_{0}=2 \mathrm{GeV}$, the ansatz in (26) and (27) gives a good description of the data for these form factors. For gluons we take a slightly simpler form than (26) and set

$$
f_{g}(x)=\alpha_{g}^{\prime}(1-x)^{2} \ln \frac{1}{x}+B_{g}(1-x)^{2} .
$$

For the parameters we take

$$
\alpha_{g}^{\prime}=0.164 \mathrm{GeV}^{-2}, \quad B_{g}=1.2 \mathrm{GeV}^{-2}
$$

so as to match recent $\mathrm{H} 1$ data on $J / \Psi$ photoproduction, whose $t$ dependence is well fitted by [30]

$$
\frac{d \sigma}{d t} \propto \exp \left[\left(b_{0}+4 \alpha_{g}^{\prime} \ln \frac{W_{\gamma p}}{W_{0}}\right) t\right]
$$

with central values $b_{0}=4.63 \mathrm{GeV}^{-2}$ and $\alpha_{g}^{\prime}=0.164 \mathrm{GeV}^{-2}$ for $W_{0}=90 \mathrm{GeV}$. To connect (31) with (29) we have used the approximate relation $d \sigma / d t \propto\left|H^{g}(\xi, \xi, t)\right|^{2}$, which is obtained when only keeping the imaginary part of the tree-level amplitude, where $2 \xi=\left(M_{J / \Psi} / W_{\gamma p}\right)^{2}$ in terms of the $\gamma p$ c.m. energy. With the ansatz (23) one approximately has $H^{g}(\xi, \xi, t) \propto \exp \left[t f_{g}(2 \xi)\right]$ for the $t$ dependence of the GPD [13.

Whereas information on valence quark GPDs can be obtained from the sum rules (28) and information on gluon GPDs from $J / \Psi$ production, almost nothing is so far known about the $t$ dependence of GPDs for antiquarks. As a simple ansatz we shall take their slope functions equal to those in the valence sector,

$$
f_{\bar{u}}=f_{u_{v}}, \quad f_{\bar{d}}=f_{d_{v}}, \quad f_{\bar{s}}=f_{d_{v}},
$$

bearing in mind that it remains an outstanding task to develop more realistic models.

\subsection{Nucleon helicity-flip distributions}

The nucleon helicity-flip distributions $E^{q}$ and $E^{g}$ are less-well known than their counterparts $H^{q}$ and $H^{g}$, because their values at $\xi=0$ and $t=0$ cannot be measured in inclusive processes and are thus subject to considerable uncertainty.

The model described in this subsection refers to a scale of $\mu_{0}=2 \mathrm{GeV}$. We make a double distribution based ansatz

$$
\begin{aligned}
E^{q(+)}(x, \xi, t) & =\int_{-1}^{1} d \beta \int_{-1+|\beta|}^{1-|\beta|} d \alpha \delta(x-\beta-\xi \alpha) h^{(2)}(\beta, \alpha) E^{q(+)}(\beta, 0, t) \\
E^{g}(x, \xi, t) & =\int_{-1}^{1} d \beta \int_{-1+|\beta|}^{1-|\beta|} d \alpha \delta(x-\beta-\xi \alpha) h^{(2)}(\beta, \alpha) E^{g}(\beta, 0, t)
\end{aligned}
$$


as in (23), and for $x>0$ set

$$
\begin{aligned}
E^{q(+)}(x, 0, t) & =e_{q_{v}}(x) \exp \left[\operatorname{tg}_{q_{v}}(x)\right]+2 e_{\bar{q}}(x) \exp \left[\operatorname{tg} g_{\bar{q}}(x)\right], \\
E^{g}(x, 0, t) & =x e_{g}(x) \exp \left[\operatorname{tg}_{g}(x)\right],
\end{aligned}
$$

with the corresponding values for $x<0$ determined by the symmetry properties of the distributions. For the forward limit of the valence distribution we take

$$
e_{q_{v}}(x)=\kappa_{q} N\left(\alpha_{v}, \beta_{q_{v}}\right) x^{-\alpha_{v}}(1-x)^{\beta_{q_{v}}}
$$

whose normalization factor

$$
N(\alpha, \beta)=\frac{\Gamma(2-\alpha+\beta)}{\Gamma(1-\alpha) \Gamma(1+\beta)}
$$

ensures the sum rules

$$
\kappa_{q}=\int_{-1}^{1} d x E^{q}(x, 0,0)=\int_{0}^{1} d x e_{q_{v}}(x),
$$

where $\kappa_{u} \approx 1.67$ and $\kappa_{d} \approx-2.03$ are the contributions of $u$ and $d$ quarks to the anomalous magnetic moment of the proton. For the functions controlling the $t$ dependence we take the same form as in $(\underline{26})$,

$$
g_{q_{v}}(x)=\alpha_{v}^{\prime}(1-x)^{3} \ln \frac{1}{x}+D_{q_{v}}(1-x)^{3}+C_{q_{v}} x(1-x)^{2} .
$$

With the parameters $\alpha_{v}=0.55, \alpha_{v}^{\prime}=0.9 \mathrm{GeV}^{-2}$ and

$$
\begin{array}{lll}
\beta_{u}=3.99, & C_{u_{v}}=1.22 \mathrm{GeV}^{-2}, & D_{u_{v}}=0.38 \mathrm{GeV}^{-2}, \\
\beta_{d}=5.59, & C_{d_{v}}=2.59 \mathrm{GeV}^{-2}, & D_{d_{v}}=-0.75 \mathrm{GeV}^{-2},
\end{array}
$$

from [28] one obtains a good fit to the electromagnetic Pauli form factors of proton and neutron via the generalization of the sum rule (37) to finite $t$.

For the forward limit of the distributions of antiquarks and gluons we make the same simple ansatz as in (35),

$$
e_{\bar{q}}(x)=k_{\bar{q}} x^{-\alpha_{\bar{q}}}(1-x)^{\beta_{\bar{q}}}, \quad e_{g}(x)=k_{g} x^{-\alpha_{g}}(1-x)^{\beta_{g}},
$$

and for the $t$ dependence in the gluon sector we set

$$
g_{g}(x)=\alpha_{g}^{\prime}(1-x)^{2} \ln \frac{1}{x}+D_{g}(1-x)^{2},
$$

in analogy to the form (29) we used for $H^{g}$. We presently have not no phenomenological information on these distributions, but two theoretical constraints. There is a condition that ensures positive semidefinite densities of partons in the transverse plane [31, which with our ansatz for the GPDs reads 28 ]

$$
\begin{aligned}
& {\left[\frac{e_{\bar{q}}(x)}{\bar{q}(x)}\right]^{2} \leq 8 \mathrm{e} m_{p}^{2}\left[\frac{g_{\bar{q}}(x)}{f_{\bar{q}}(x)}\right]^{3}\left[f_{\bar{q}}(x)-g_{\bar{q}}(x)\right],} \\
& {\left[\frac{e_{g}(x)}{g(x)}\right]^{2} \leq 8 \mathrm{e} m_{p}^{2}\left[\frac{g_{g}(x)}{f_{g}(x)}\right]^{3}\left[f_{g}(x)-g_{g}(x)\right]}
\end{aligned}
$$


if we neglect for simplicity the polarized antiquark and gluon distributions compared with the unpolarized ones. On the other hand we have the sum rule

$$
\begin{aligned}
0 & =\int_{0}^{1} d x E^{g}(x, 0,0)+\sum_{q} \int_{-1}^{1} d x x E^{q}(x, 0,0) \\
& =\int_{0}^{1} d x x e^{g}(x)+\sum_{q} \int_{0}^{1} d x x\left[e_{q_{v}}(x)+2 e_{\bar{q}}(x)\right]
\end{aligned}
$$

following from the conservation of the energy-momentum tensor. For the parameters in (41) we take

$$
\alpha_{g}^{\prime}=0.164 \mathrm{GeV}^{-2}, \quad D_{g}=1.08 \mathrm{GeV}^{-2},
$$

with $\alpha_{g}^{\prime}$ as in (30) and $D_{g}$ slightly smaller than its counterpart $B_{g}$ for $H^{g}$, so that the positivity condition (42) can be fulfilled. Assuming a similar small- $x$ behavior of the distributions for proton helicity-flip and non-flip, we take in (40) the values $\alpha_{\bar{q}}=1.25$ and $\alpha_{g}=1.10$, which we obtain when fitting the CTEQ6M distributions to a power law in the $x$ range from $10^{-4}$ to $10^{-3}$.

Since it turns out that the transverse target polarization asymmetry in $\rho$ production is very sensitive to the details of the helicity-flip distributions, we will explore two model scenarios in our numerical studies:

1. a scenario where the sea quark distributions $e_{\bar{q}}$ behave similarly to the valence distributions $e_{q_{v}}$. For the $t$ dependence we then take $g_{\bar{u}}(x)=g_{u_{v}}(x)$ and $g_{\bar{d}}(x)=g_{d_{v}}(x)$. The parameters $k_{\bar{q}}$ in (40) are taken such that second moments at $t=0$ fulfill

$$
\frac{\int_{0}^{1} d x x e_{\bar{q}}(x)}{\int_{0}^{1} d x x e_{q_{v}}(x)}=\frac{\int_{0}^{1} d x x \bar{q}(x)}{\int_{0}^{1} d x x q_{v}(x)}
$$

for $q=u, d$, where the ratio on the r.h.s. is taken from the CTEQ6M parameterization at $\mu=$ $2 \mathrm{GeV}$. Its value is 0.095 for $u$ and 0.30 for $d$ quarks. This fixes the values of $k_{\bar{q}} N^{-1}\left(\alpha_{\bar{q}}-1, \beta_{\bar{q}}\right)$ with $N$ given in (36) $)$. For the strange distribution we set $e_{s}=e_{\bar{s}}=0$, and $k_{g} N^{-1}\left(\alpha_{g}-1, \beta_{g}\right)$ is then fixed by the sum rule (43).

The powers $\beta_{\bar{q}}$ and $\beta_{g}$ controlling the large- $x$ behavior are finally taken to have the smallest values for which the positivity condition (42) holds in the range $x<0.9$ (for higher $x$ even the unpolarized densities are so uncertain that we do not insist on the positivity conditions to be fulfilled).

2. a scenario where $e_{\bar{q}}$ behaves similarly to the gluon distribution $e_{g}$. The $t$ dependence is now modeled by taking $g_{\bar{q}}(x)=g_{g}(x)$ for $q=u, d, s$. For the second moments we impose

$$
\frac{\int_{0}^{1} d x x e_{\bar{q}}(x)}{\int_{0}^{1} d x x e_{g}(x)}=\frac{\int_{0}^{1} d x x \bar{q}(x)}{\int_{0}^{1} d x x g(x)}
$$

for the three light quark flavors, where with the CTEQ6M distributions the r.h.s. is equal to $0.064,0.083,0.036$ for $u, d, s$, respectively. We now have a nonzero $e_{s}=e_{\bar{s}}$. The values of $k_{\bar{q}} N^{-1}\left(\alpha_{\bar{q}}-1, \beta_{\bar{q}}\right)$ and $k_{g} N^{-1}\left(\alpha_{g}-1, \beta_{g}\right)$ are taken to fulfill both (46) and (43), and the powers $\beta_{\bar{q}}, \beta_{g}$ are set to the minimal values for which positivity holds in the range $x<0.9$.

The parameters resulting from this modeling procedure are collected in Table 1 , and the distributions at $\xi=0$ and $t=0$ for model 1 are shown in Fig. 2 . 
Table 1: Parameters in the ansatz (40) for different parton species $a$ in the two models described in the text. The values for valence quarks apply to both models, with normalization parameters given by $k_{q_{v}}=\kappa_{q} N\left(\alpha_{v}, \beta_{q_{v}}\right)$ according to (35). The last line gives the second Mellin moment at $\mu=2 \mathrm{GeV}$ in the forward limit.

\begin{tabular}{|c|c|c|c|c|c|c|c|c|c|}
\hline & \multirow[b]{2}{*}{$u_{v}$} & \multirow[b]{2}{*}{$d_{v}$} & \multicolumn{3}{|c|}{ model 1} & \multicolumn{4}{|c|}{ model 2} \\
\hline & & & $\bar{u}$ & $\bar{d}$ & $g$ & $\bar{u}$ & $\bar{d}$ & $\bar{s}$ & $g$ \\
\hline$\alpha_{a}$ & 0.55 & 0.55 & 1.25 & 1.25 & 1.10 & 1.25 & 1.25 & 1.25 & 1.10 \\
\hline$\beta_{a}$ & 3.99 & 5.59 & 9.6 & 9.2 & 6.7 & 7.6 & 6.5 & 5.5 & 2.5 \\
\hline$k_{a}$ & 1.71 & -2.36 & 0.06 & -0.18 & 0.26 & -0.0016 & -0.0018 & -0.0007 & -0.017 \\
\hline $\int_{0}^{1} d x x e_{a}(x)$ & 0.138 & -0.130 & 0.013 & -0.039 & 0.044 & -0.0004 & -0.0005 & -0.0002 & -0.0059 \\
\hline
\end{tabular}
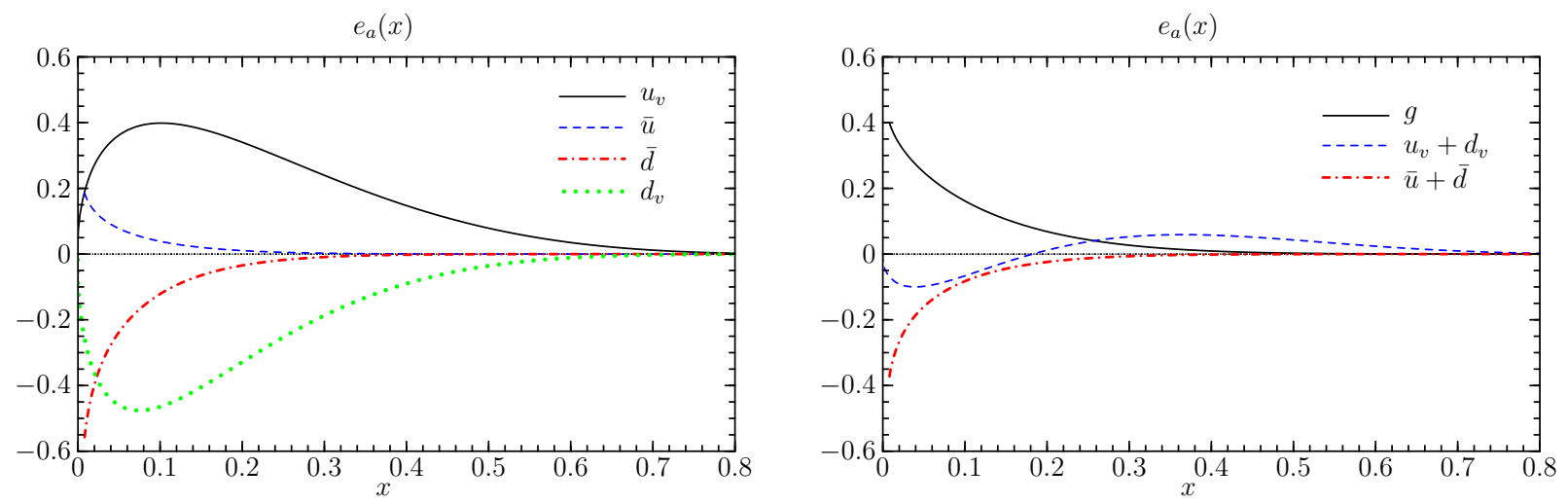

Figure 2: The forward limits $e_{a}(x)$ of the nucleon helicity-flip distributions at $\mu=2 \mathrm{GeV}$ for different parton species $a$ in model 1 .

We find that in model 2, both sea quark and gluon distributions are nearly zero (so that we do not attach importance to the unrealistically small value of $\beta_{g}$ obtained with our above procedure). Their smallness can be traced back to the small value of the flavor singlet integral

$$
\int_{0}^{1} d x x\left[e_{u_{v}}(x)+e_{d_{v}}(x)\right]=0.008
$$

in the valence sector of our ansatz. In model 2 , the distributions $e_{\bar{q}}$ and $e_{g}$ have the same sign as a consequence of (46) and due to the sum rule (43) can only be tiny. Somewhat larger distributions for sea quarks and gluons are obtained in model 1, where they have opposite sign because of (45).

We note that the parameters (39) we have taken for the valence part of $E^{q}$ are by no means precisely determined by a fit to the Pauli form factors: alternative fits in [28] gave a similarly good description of the form factor data, with some variation of the resulting value of the integral in (47). Nevertheless, any model where $e_{u_{v}}$ and $e_{d_{v}}$ have similar shapes and no zeroes in $x$ will yield rather small values of this integral, given the strong cancellation between $u$ and $d$ quark contributions in the moment $\int_{0}^{1} d x\left[e_{u_{v}}(x)+e_{d_{v}}(x)\right]=\kappa_{u}+\kappa_{d} \approx-0.36$. It would be interesting to explore how much the integral (47) and as a consequence the sea quark and gluon distributions can vary in realistic models, but such an investigation is beyond the scope of this work. 
We end this section by quoting the values for the total angular momentum carried by quarks and antiquarks of a given flavor in our model, given by

$$
J_{q}=\frac{1}{2} \int_{-1}^{1} d x x\left[H^{q}(x, 0,0)+E^{q}(x, 0,0)\right]
$$

according to Ji's sum rule 2]. With the parameters in Table 1 and the CTEQ6M distributions we find

$$
\begin{array}{ll}
J_{u}=0.25, & J_{d}=-0.01, \\
J_{u}=0.24, & J_{d}=0.03
\end{array}
$$

at the scale $\mu=2 \mathrm{GeV}$ of our model. We note that this is in rather good agreement with the results of recent lattice calculations, with $J_{u}=0.214(16)$ and $J_{d}=-0.001(16)$ reported in [32], and $J_{u}=0.33(2)$ and $J_{d}=-0.02(2)$ in $[33$. Let us reiterate that with just two sets of model parameters we cannot exhaust the range of possible scenarios but only provide two representatives that are consistent with presently known constraints. As just discussed, the relative smallness of sea quark and gluon distributions compared with the nucleon helicity conserving case should however be typical of a rather wide class of models.

\section{Vector meson production at small $x_{B}$}

We now study numerically the importance of NLO corrections in vector meson production. Here and in the following sections we use the two-loop strong coupling for $n_{f}=3$ flavors with a QCD scale parameter $\Lambda^{(3)}=226 \mathrm{MeV}$. This value corresponds to $\Lambda^{(4)}=326 \mathrm{MeV}, \Lambda^{(5)}=372 \mathrm{MeV}$ and to $\alpha_{s}^{(5)}\left(M_{Z}\right)=0.118$ when matching at $m_{c}=1.3 \mathrm{GeV}$ and $m_{b}=4.5 \mathrm{GeV}$, which are the values used in the CTEQ6M parton analysis [29]. We also take $n_{f}=3$ fixed in the evolution and the hard-scattering kernels. Taking $n_{f}=4$ with massless charm or $n_{f}=5$ with massless charm and bottom would not be a good approximation for the rather moderate values of $Q^{2}$ we will discuss for fixed-target kinematics. On the other hand, taking $n_{f}=3$ and neglecting charm altogether is admittedly not a good approximation for the larger $Q^{2}$ relevant in collider kinematics. However, with $\alpha_{s}^{(3)}=0.164$ compared to $\alpha_{s}^{(5)}=0.178$ at $\mu=10 \mathrm{GeV}$ we expect that this inaccuracy will not affect the conclusions at high $Q^{2}$ we shall draw from our studies.

We have performed the evolution of the GPDs at LO using the momentum-space evolution code of [34. As explained in Sect. 2, taking LO evolution together with the NLO hard-scattering kernels is sufficient to obtain scale independence of the process amplitude up to uncalculated corrections of order $\alpha_{s}^{3}$. With the input scale of evolution not taken too small, NLO evolution effects should be rather moderate at the $Q^{2}$ values relevant in fixed-target kinematics, whereas our general conclusions for high $Q^{2}$ and small $x_{B}$ will again not depend on this level of detail. We note that the NLO kernels in momentum space are available in the literature [35, but their considerable length makes it difficult to implement them in a fast numerical evaluation. For including NLO effects in the evolution it should be more efficient to use the Mellin space approach recently followed for deeply virtual Compton scattering in [5].

Here and in the following section we consider the convolutions of hard-scattering kernels with GPDs at $t=0$. For nonzero $\xi=x_{B} /\left(2-x_{B}\right)$ this should be understood in the sense of an analytic continuation, since the physical region for meson production is $-t \geq 4 m_{p}^{2} \xi^{2} /\left(1-\xi^{2}\right)$ in Bjorken kinematics. To explore the importance of NLO corrections we do not see this as a shortcoming.

Let us start our discussion with the gluon and quark singlet sector. Here and in following we shall always present the convolutions (19) for Gegenbauer index $n=0$ unless indicated otherwise. In Fig. 3 

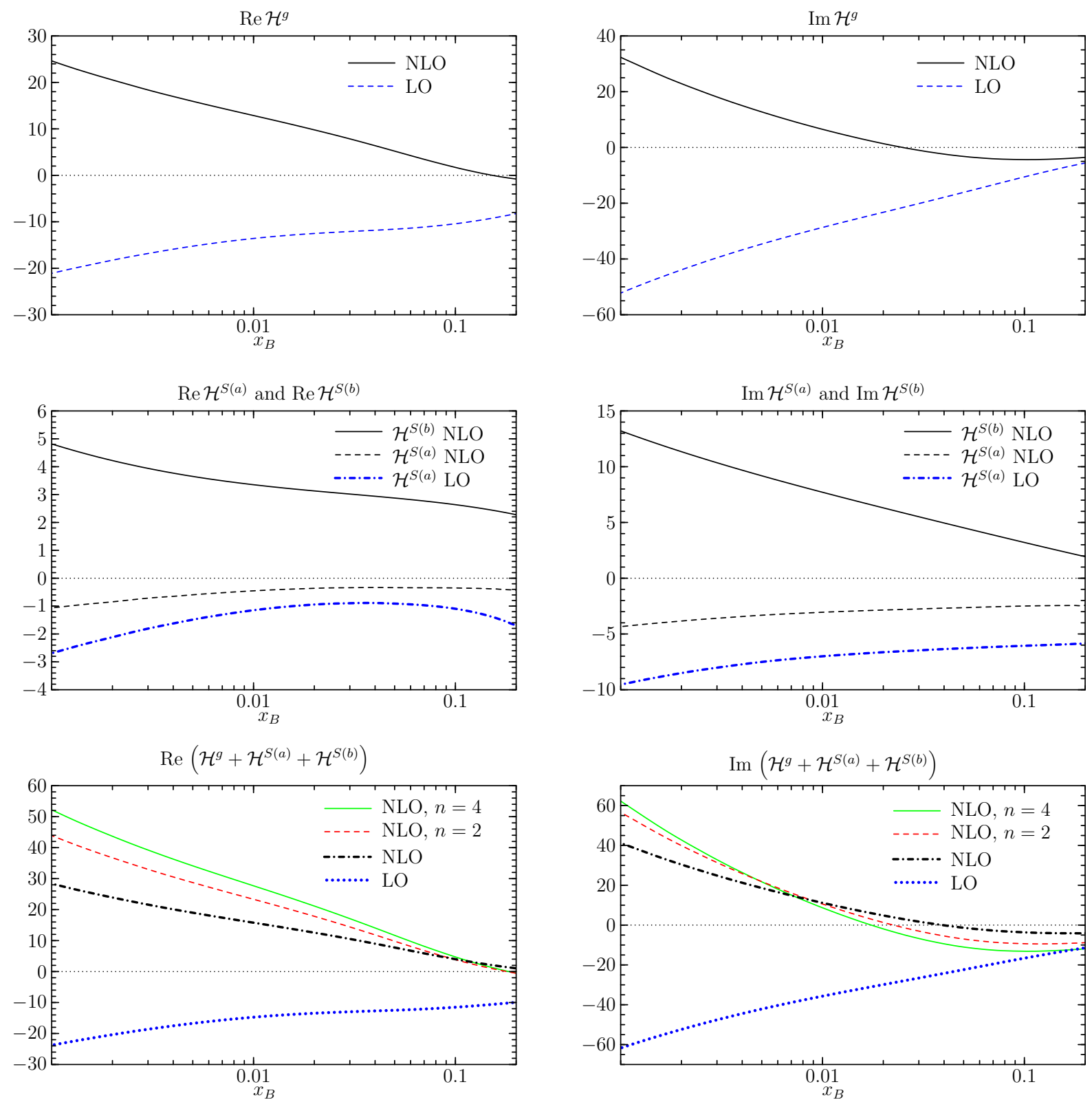

Figure 3: LO and NLO terms of the convolutions in the gluon and quark singlet sector at $Q=4 \mathrm{GeV}$. The scales are set to $\mu_{R}=\mu_{G P D}=\mu_{D A}=Q$. The NLO terms are for Gegenbauer index $n=0$ unless specified explicitly. Here and in the following plots the label "NLO" denotes the $O\left(\alpha_{s}^{2}\right)$ part of the convolutions, whereas the sum of $O\left(\alpha_{s}\right)$ and $O\left(\alpha_{s}^{2}\right)$ terms is labeled by "LO+NLO". 

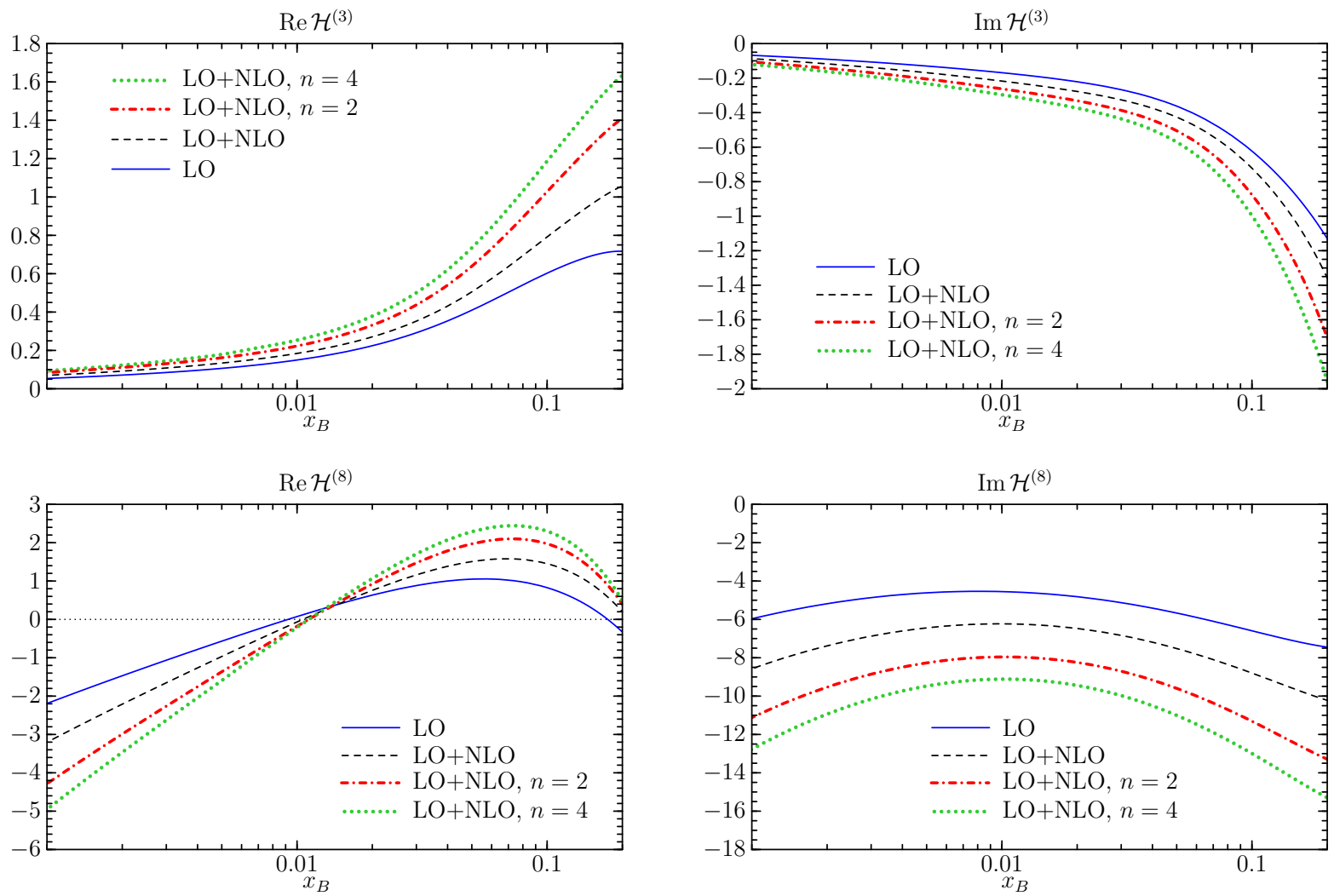

Figure 4: LO terms and the sum of LO and NLO terms of the convolutions in the quark non-singlet sector at $Q=4 \mathrm{GeV}$, with $\mu_{R}=\mu_{G P D}=\mu_{D A}=Q$. 

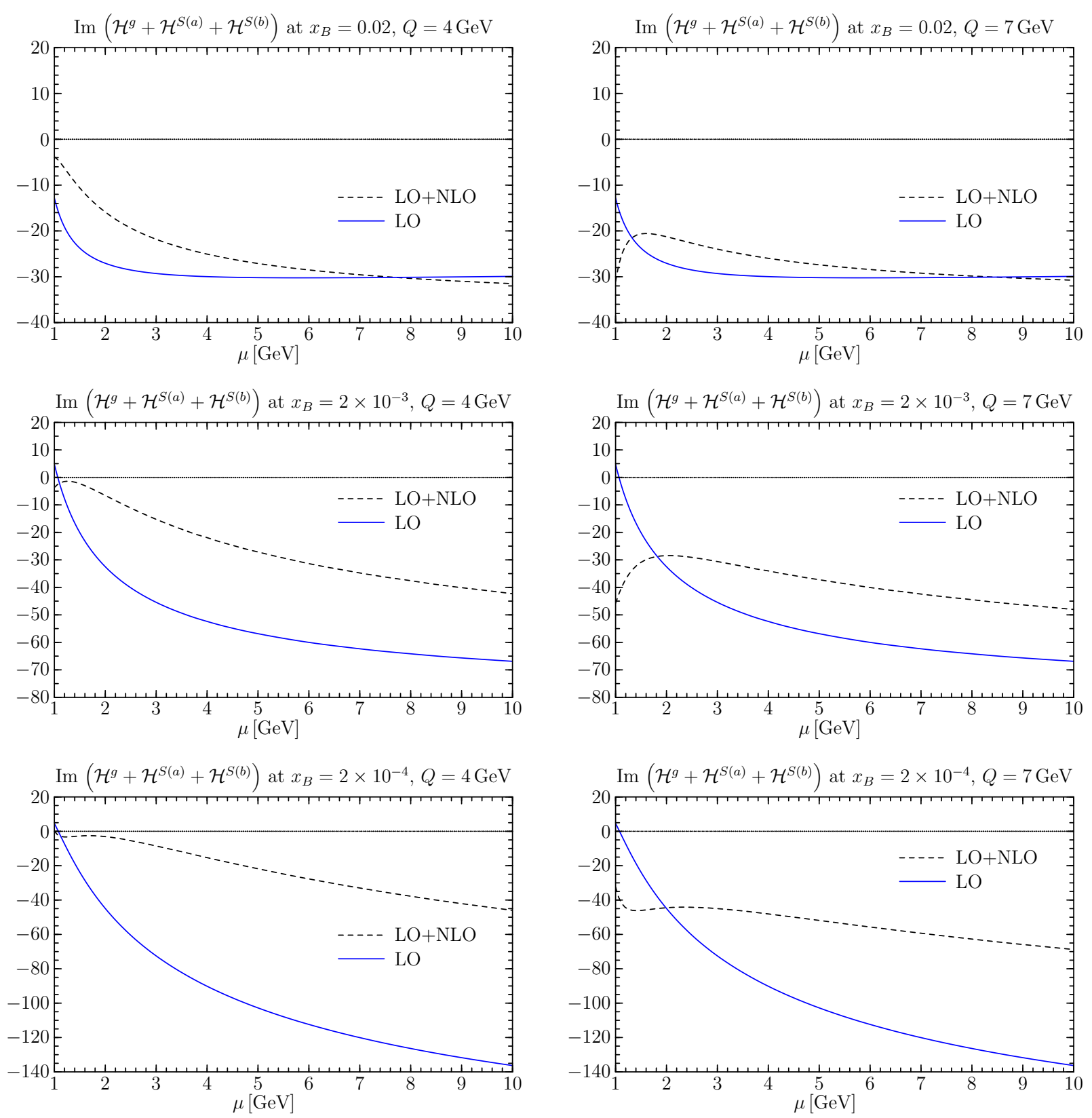

Figure 5: Dependence on the common scale $\mu=\mu_{R}=\mu_{G P D}$ for the sum of convolutions in the gluon and quark singlet sector. 


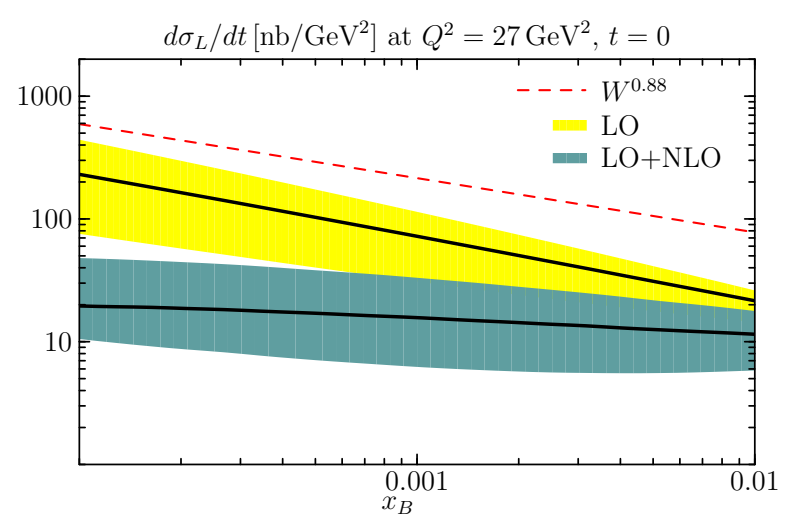

Figure 6: Cross section for $\gamma^{*} p \rightarrow \rho p$ with longitudinal photon polarization. Bands correspond to the range $Q / 2<\mu<2 Q$ and solid lines to $\mu=Q$. We also show the power-law behavior $\sigma \propto W^{0.88}$ (with arbitrary normalization) obtained from a fit to data in the range $0.001 \lesssim x_{B} \lesssim 0.005$ [36].

we show the LO and NLO pieces of the convolutions for the scale choice $\mu_{R}=\mu_{G P D}=\mu_{D A}=Q$. The size of corrections at small $x_{B}$ is dramatic: we have large NLO corrections with opposite sign compared to the LO term for $\mathcal{H}^{g}$, and a similarly large NLO contribution from $\mathcal{H}^{S(b)}$ with sign opposite to the LO result for $\mathcal{H}^{S(a)}$. In the sum of gluon and quark singlet terms, the NLO corrections drastically reduce the LO result or even lead to a change of sign between LO and the sum of LO and NLO results. We also observe that for higher Gegenbauer index the NLO corrections tend to be even more important. Note that the LO term of the convolutions is the same for all $n$ as can be seen from (19) and (20). The size of NLO corrections in $\mathcal{H}^{S(a)}$ is comparatively moderate, at least for lower Gegenbauer moments. The same is seen for the quark non-singlet convolutions in Fig. 4, Of course, the gluon and quark singlet terms will dominate meson production at small $x_{B}$ in those channels where it is allowed by the meson quantum numbers.

In Fig. 5 we explore the influence of the scale choice by varying $\mu_{R}=\mu_{G P D}$ simultaneously. For $x_{B}=2 \times 10^{-3}$ we find an indication for the onset of perturbative stability at $Q=7 \mathrm{GeV}$ but not yet at $Q=4 \mathrm{GeV}$. For $x_{B}=2 \times 10^{-2}$ the situation is less severe, with moderate corrections in a wide $\mu$ range already at $Q=4 \mathrm{GeV}$. In contrast, when going down to $x_{B}=2 \times 10^{-4}$ we find very large corrections even at $Q=7 \mathrm{GeV}$. We have checked that the conclusions in the respective kinematics do not change when we vary $\mu_{G P D}$ while keeping $\mu_{R}=Q$ fixed.

Figure 6 shows how the perturbative instability we observed in the convolutions affects the longitudinal cross section for $\rho$ production. Here we have taken the asymptotic form of the meson distribution amplitude, i.e. set $a_{n}=0$ for $n \geq 2$. In the NLO result for the cross section we have squared the coherent sum of LO and NLO terms in the process amplitude 2 i.e. we have taken $\left|\mathcal{M}_{\mathrm{LO}}+\mathcal{M}_{\mathrm{NLO}}\right|^{2}$. We see that the NLO corrections severely decrease the LO result. As a consequence of the cancellations between LO and NLO contributions, the scale dependence of the cross section does not decrease. We also show in the figure the power-law behavior $\sigma \propto W^{0.88}$ obtained from a fit to data in the range $0.001 \lesssim x_{B} \lesssim 0.005$ [36]. As observed in [13], a double distribution model with the CTEQ6M distributions as input lead to a rather good description of this experimentally observed energy dependence

\footnotetext{
${ }^{2}$ We thus keep terms of $O\left(\alpha_{s}^{3}\right)$ in the cross section, although the accuracy of the NLO calculation is only up to $O\left(\alpha_{s}^{2}\right)$. This should not be seen as a problem, as it will not make a considerable difference in situations where perturbative corrections are moderate, whereas in situations where NLO corrections are huge we would neither trust the cross section with or without the partially included $O\left(\alpha_{s}^{3}\right)$ terms.
} 

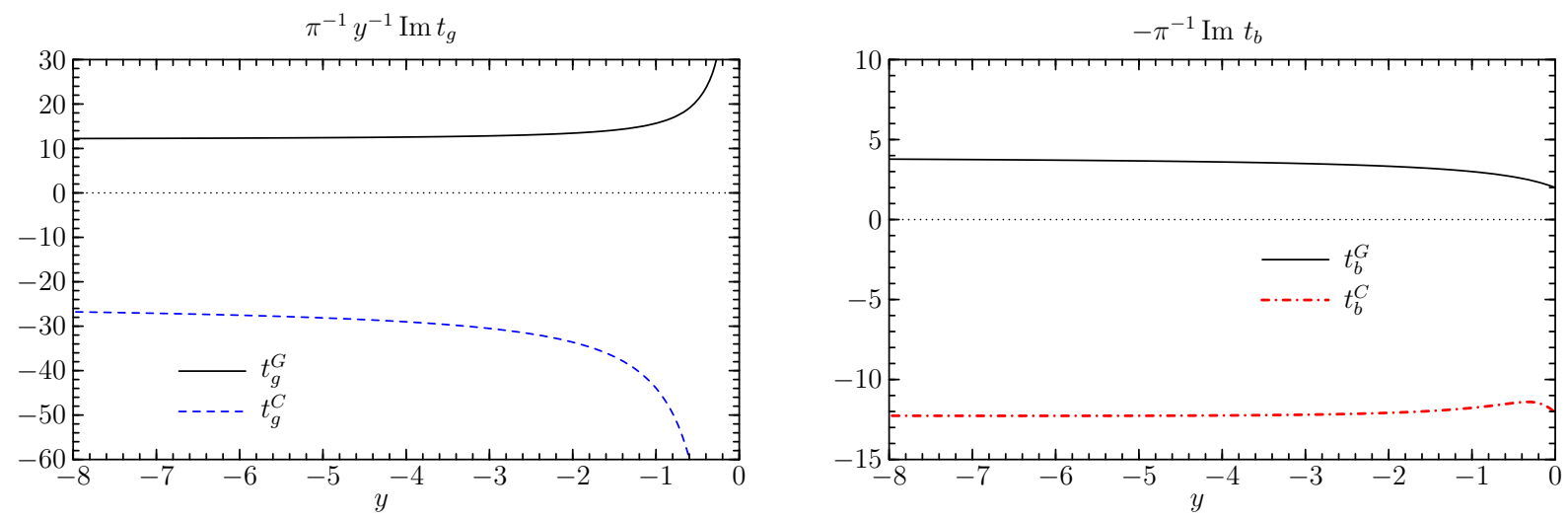

Figure 7: The factorization scale dependent and independent terms of $\operatorname{Im} t_{g, n}$ and $\operatorname{Im} t_{b, n}$ as specified in (54), shown for $n=0$ and $y<0$.

if the cross section is evaluated at LO. With the strong cancellations from the $O\left(\alpha_{s}^{2}\right)$ corrections, one obtains an NLO result whose energy behavior is much too weak.

Let us discuss how the huge size of corrections can be understood at an analytical level, following the line of argument given in [11, 37. Using (81) and (82) we can approximate the hard-scattering kernels for large negative $y$ as

$$
\begin{array}{ll}
\frac{1}{\pi} \operatorname{Im} \mathcal{I}_{g}(z, y)=4 C_{A}\left[\ln (z \bar{z})+\ln \frac{Q^{2}}{\mu_{G P D}^{2}}\right] y+O(1), & \operatorname{Re} \mathcal{I}_{g}(z, y)=O(1), \\
\frac{1}{\pi} \operatorname{Im} \mathcal{I}_{b}(z, y)=4\left[1-\ln (z \bar{z})-\ln \frac{Q^{2}}{\mu_{G P D}^{2}}\right]+O\left(y^{-1}\right), & \operatorname{Re} \mathcal{I}_{b}(z, y)=O\left(y^{-1}\right),
\end{array}
$$

where here and in the following the order of corrections is given up to powers of $\ln \bar{y}$. The quark non-singlet kernel is subleading compared with the pure singlet one,

$$
\frac{\mathcal{I}_{a}(\bar{z}, y)}{y} \sim \frac{\mathcal{I}_{a}(z, \bar{y})}{\bar{y}} \sim O\left(y^{-1}\right)
$$

where we have divided $\mathcal{I}_{a}(\bar{z}, y)$ by $y$ corresponding to the prefactor in the complete kernel (10). From (50) we readily obtain

$$
\begin{aligned}
& \frac{1}{\pi} \operatorname{Im} t_{g, n}(y)=-4 C_{A}\left[c_{n}-\ln \frac{Q^{2}}{\mu_{G P D}^{2}}\right] y+O(1), \\
& \frac{1}{\pi} \operatorname{Im} t_{b, n}(y)=4\left[c_{n}+1-\ln \frac{Q^{2}}{\mu_{G P D}^{2}}\right]+O\left(y^{-1}\right)
\end{aligned}
$$

with constants

$$
c_{0}=2, \quad c_{2}=\frac{11}{3} \approx 3.7, \quad c_{4}=\frac{137}{30} \approx 4.6, \quad c_{n}=-\int_{0}^{1} d z \ln (z \bar{z}) C_{n}^{3 / 2}(2 z-1)
$$

that increase with the Gegenbauer index $n$. In Fig. 7 we show for the case $n=0$ that these approximations become very good for increasing $|y|$, where we have decomposed the exact kernels as

$$
\operatorname{Im} t_{g, n}(y)=\operatorname{Im} t_{g, n}^{C}(y)+\operatorname{Im} t_{g, n}^{G}(y) \ln \frac{Q^{2}}{\mu_{G P D}^{2}},
$$




$$
\operatorname{Im} t_{b, n}(y)=\operatorname{Im} t_{b, n}^{C}(y)+\operatorname{Im} t_{b, n}^{G}(y) \ln \frac{Q^{2}}{\mu_{G P D}^{2}} .
$$

Let us now rewrite the convolutions of kernels and GPDs in terms of the variable $\omega=x / \xi$,

$$
\begin{aligned}
& \operatorname{Im}\left[\mathcal{H}_{n}^{g}+\mathcal{H}_{n}^{S(b)}\right]=-6 \alpha_{s}\left[\pi H^{g}(\xi, \xi, t)+\frac{\alpha_{s}}{4 \pi} \operatorname{Im} \int_{0}^{1 / \xi} d \omega\right. \\
& \left.\quad \times\left\{\frac{2}{1-\omega-i \epsilon} t_{g, n}\left(\frac{1-\omega-i \epsilon}{2}\right) \frac{H^{g}(\omega \xi, \xi, t)}{1+\omega}-C_{F} t_{b, n}\left(\frac{1-\omega-i \epsilon}{2}\right) \xi H^{S}(\omega \xi, \xi, t)\right\}\right] .
\end{aligned}
$$

For $\omega \geq \omega_{0}$ with some $\omega_{0} \gg 1$ we can use the approximation (52) of the hard-scattering kernels, and further approximate $1+\omega \approx \omega$ in the first term on the second line. This gives

$$
\begin{aligned}
& -\frac{1}{6 \pi \alpha_{s}} \operatorname{Im}\left[\mathcal{H}_{n}^{g}+\mathcal{H}_{n}^{S(b)}\right] \approx H^{g}(\xi, \xi, t)+\frac{\alpha_{s}}{\pi} \int_{0}^{\omega_{0}} d \omega \ldots \\
& \quad-\frac{\alpha_{s}}{\pi} \int_{\omega_{0}}^{1 / \xi} d \omega\left\{C_{A}\left[c_{n}-\ln \frac{Q^{2}}{\mu_{G P D}^{2}}\right] \frac{H^{g}(\omega \xi, \xi, t)}{\omega}+C_{F}\left[c_{n}+1-\ln \frac{Q^{2}}{\mu_{G P D}^{2}}\right] \xi H^{S}(\omega \xi, \xi, t)\right\},
\end{aligned}
$$

where the integral over $\omega$ on the first line is to be taken with the unapproximated integrand from (55). It grows with $\xi$ like $H^{g}(\omega \xi, \xi, t)$ or $\xi H^{S}(\omega \xi, \xi, t)$ but lacks the enhancement due to the upper limit $1 / \xi$ of the integral on the second line. Restricting our discussion to $t=0$ for simplicity, we can for sufficiently large $\omega$ neglect the effect of skewness in the GPDs and then have

$$
H^{g}(\omega \xi, \xi, 0) \approx \omega \xi g(\omega \xi), \quad H^{S}(\omega \xi, \xi, 0) \approx S(\omega \xi)=\sum_{q}[q(\omega \xi)+\bar{q}(\omega \xi)]
$$

where $S(x)$ is the usual quark singlet distribution. In a very rough approximation one may treat $x g(x)$ and $x S(x)$ as constant at small $x$. In (56) one then has loop integrals $\int d \omega / \omega$ for both the gluon and the quark term, which generate large logarithms $\ln \left(\omega_{0} \xi\right)$ for $1 / \xi \gg \omega_{0}$. These logarithms are of BFKL type and correspond to graphs with $t$-channel gluon exchange in the hard-scattering kernel, such as those for $T_{b}$ and $T_{g}$ in Fig. 1.

In a phenomenologically more realistic approximation one has $x g(x) \approx a x^{-\lambda}$ at small $x$ and a similar behavior with different values of $a$ and $\lambda$ for $x S(x)$. This gives

$$
\int_{\omega_{0}}^{1 / \xi} d \omega \frac{H^{g}(\omega \xi, \xi, 0)}{\omega} \approx a \xi^{-\lambda} \int_{\omega_{0}}^{1 / \xi} d \omega \omega^{-\lambda-1} \approx \frac{a}{\lambda}\left(\omega_{0} \xi\right)^{-\lambda}
$$

for $1 / \xi \gg \omega_{0}$, when the bulk of the integral comes from the region where the small- $x$ approximation of the gluon density is valid. With $\lambda$ being rather small for the gluon distribution in a wide range of the factorization scale, the term (58) has the same power behavior $\xi^{-\lambda}$ as the Born term $H^{g}(\xi, \xi, 0)$ in (56) but is numerically enhanced by $1 / \lambda$. A contribution analogous to (58) is obtained from the quark singlet term in (56) and comes with a similar enhancement.

Concerning the choice of factorization scale, it is clear that the size of the corrections in (56) is decreased if $\mu_{G P D}$ is taken smaller than $Q$. It is also clear that no scale choice can eliminate both the gluon and quark singlet contribution in this expression. To make at least the gluon term for $n=0$ disappear one needs $\mu_{G P D}^{2}=e^{-2} Q^{2} \approx 0.14 Q^{2}$. For a wide range of $Q^{2}$ this is outside the perturbative region or at least so low that the quark singlet distribution has a rather small power $\lambda$ and can thus give important corrections. We note that previous analyses of vector meson production at small $x_{B}$ have argued for a factorization scale well below $Q^{2}$, based on different estimates of the typical virtualities in the leading-order graphs [15, 38. We also note that the $\mu_{R}$ dependent term $\beta_{0} \ln \left(\mu_{R}^{2} / \mu_{G P D}^{2}\right)$ in the gluon kernel (11) does not appear in the approximation (50) which dominates the convolutions at small $x_{B}$. The choice of $\mu_{R}$ can thus not cure the huge NLO corrections we have discussed. 


\section{$5 \quad$ Vector meson production at moderate to large $x_{B}$}

Let us now investigate the NLO corrections in typical fixed-target kinematics, as it is accessible at HERMES, JLab and COMPASS. We take again $t=0$ and for definiteness present estimates at $Q^{2}=4 \mathrm{GeV}^{2}$. For larger $Q^{2}$, which will in particular be accessible with the JLab energy upgrade to $12 \mathrm{GeV}$, the corrections are in general smaller.

In Fig. 8 we compare the $\mathrm{LO}$ and NLO parts of the convolution integrals. In the gluon sector we find no simple picture, with relative corrections that are typically moderate but become large for $\operatorname{Re} \mathcal{H}^{g}$ at smaller $x_{B}$ and for $\operatorname{Im} \mathcal{H}^{g}$ at larger $x_{B}$. For the quark singlet the situation is similar to the one in the small- $x_{B}$ region, i.e. we have rather large NLO corrections from $\mathcal{H}^{S(b)}$ with sign opposite to the LO part of $\mathcal{H}^{S(a)}$, whereas the NLO corrections in $\mathcal{H}^{S(a)}$ are smaller. Adding gluon and quark singlet contributions, we find that for $n=0$ the NLO corrections are of reasonable size for the imaginary part. For the real part at lower $x_{B}$, the corrections are however large and of opposite sign compared to the Born term. We note that the convolutions $\mathcal{H}$ satisfy a dispersion relation in $1 / x_{B}$ for fixed $Q^{2}$ and $t\left[39\right.$. In this representation their real parts at a given $x_{B}$ are sensitive to the imaginary part at smaller values of $x_{B}$, where the NLO corrections rapidly increase as we have seen in the previous section. Turning to the quark non-singlet convolutions, we see in Fig. 9 that for $n=0$ the NLO corrections are comparatively moderate for the imaginary part and larger for the real part.

Going from $n=0$ to higher Gegenbauer indices $n=2$ and $n=4$, the NLO corrections become larger, as we see in Figs. 8 and 9 and already observed at small $x_{B}$. Generically this is not unexpected, since the $z$ dependent kernels (10) contain logarithms $\ln z$ and $\ln \bar{z}$ which enhance the endpoint regions of the $z$ integration, and those endpoint regions are more prominent for higher Gegenbauer polynomials in the expansion (15). Note that according to phenomenological estimates or lattice calculations the coefficients $a_{n}$ of these polynomials are clearly smaller than $a_{0}$, so that increasing corrections to $\mathcal{H}_{n}$ for higher $n$ do not affect the sum $\sum_{n} a_{n} \mathcal{H}_{n}$ as much. We note that in the modified hard-scattering approach of Sterman et al. [14, which goes beyond the collinear approximation used in the present work, the endpoint regions in $z$ are suppressed by radiative corrections that are resummed into Sudakov form factors. As just discussed, we do not observe such a suppression in the fixed-order results analyzed here, where various positive and negative corrections compete with each other-only some of them related to the Sudakov factor. How the situation will be at higher orders is an important question, which goes beyond the scope of the present work.

Let us now take a closer look at the $\mu_{R}$ dependence of the corrections. As we explained in Sect. 2 , the pure quark singlet kernel $T_{b}$ is independent of this scale at $O\left(\alpha_{s}^{2}\right)$. According to (11) the gluon kernel $T_{g}$ depends on $\mu_{R}^{2}$ only through $\beta_{0} \ln \left(\mu_{R}^{2} / \mu_{G P D}^{2}\right)$, which originates from graphs with gluon propagator corrections such as the one shown in Fig. 10. The $\mu_{G P D}$ dependence of this term is connected with the contribution proportional to $\beta_{0}$ in the evolution kernel $V^{g g}$ for the gluon GPD, given in (92). As already pointed out in Sect. 4, the term $\beta_{0} \ln \left(\mu_{R}^{2} / \mu_{G P D}^{2}\right)$ does not contribute to the large- $|y|$ behavior of $\operatorname{Im} t_{g, n}(y)$ and is hence not relevant for the huge NLO corrections at small $x_{B}$.

For the kernel $T_{a}$ the situation is more involved. The general structure of its convolution with the quark singlet distribution $H^{S}$ can be written as

$$
\mathcal{H}_{n}^{S(a)}=\beta_{0}\left(\mathcal{H}_{n, \beta}^{S(a)}+\mathcal{H}_{n, R}^{S(a)} \ln \frac{Q^{2}}{\mu_{R}^{2}}\right)+\mathcal{H}_{n, C}^{S(a)}+\mathcal{H}_{n, G}^{S(a)} \ln \frac{Q^{2}}{\mu_{G P D}^{2}}+\mathcal{H}_{n, D}^{S(a)} \ln \frac{Q^{2}}{\mu_{D A}^{2}}
$$

with an analogous decomposition for the convolutions $\mathcal{H}_{n}^{(3)}$ and $\mathcal{H}_{n}^{(8)}$. The terms proportional to $\beta_{0}$ originate from graphs with gluon propagator corrections such as in Fig. 10, whereas the terms with subscripts $C, G, D$ do not contain $\beta_{0}$. In Fig. 11 we show the corresponding contributions for $n=0$. We see that terms multiplying $\ln \left(Q^{2} / \mu_{G P D}^{2}\right)$ are rather small, whereas those going with $\ln \left(Q^{2} / \mu_{D A}^{2}\right)$ are of course absent for $n=0$. The term $\mathcal{H}_{0, R}$ is clearly smaller than $\mathcal{H}_{0, \beta}$ and has opposite sign. 

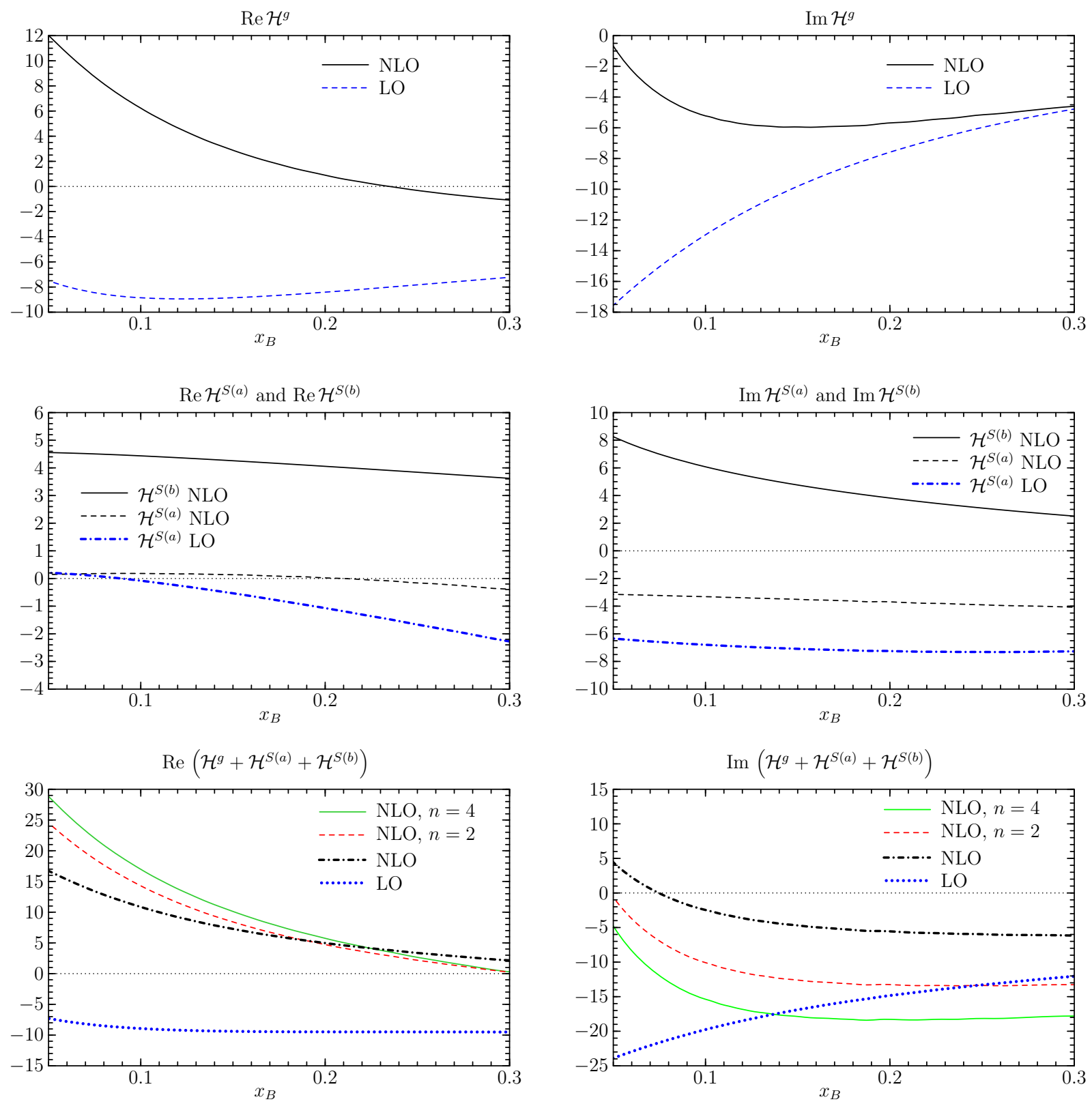

Figure 8: LO and NLO terms of the convolutions in the gluon and quark singlet sector at $Q=2 \mathrm{GeV}$, with $\mu_{R}=\mu_{G P D}=\mu_{D A}=Q$. 

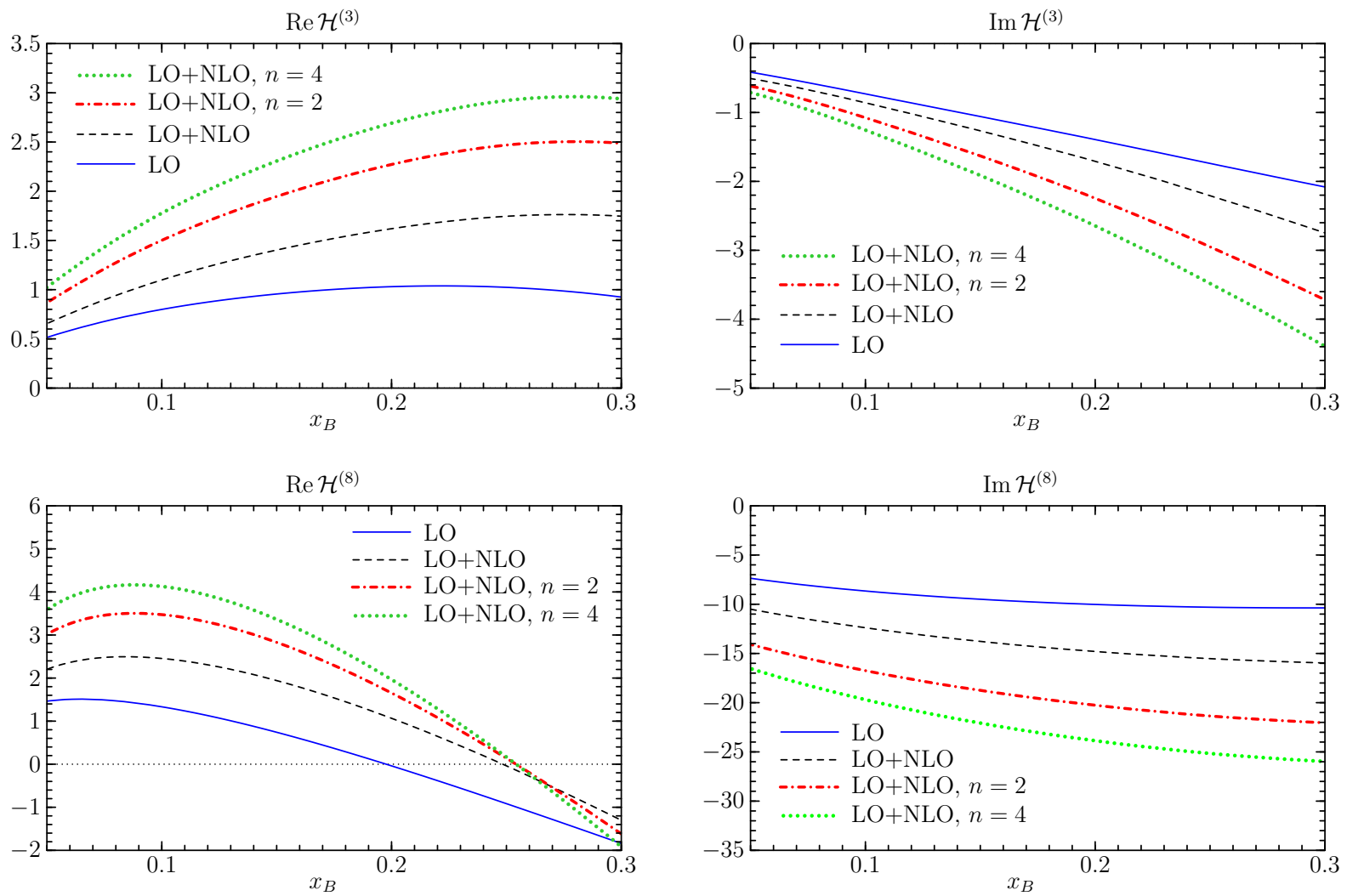

Figure 9: LO terms and the sum of LO and NLO terms of the convolutions in the quark non-singlet sector at $Q=2 \mathrm{GeV}$, with $\mu_{R}=\mu_{G P D}=\mu_{D A}=Q$.
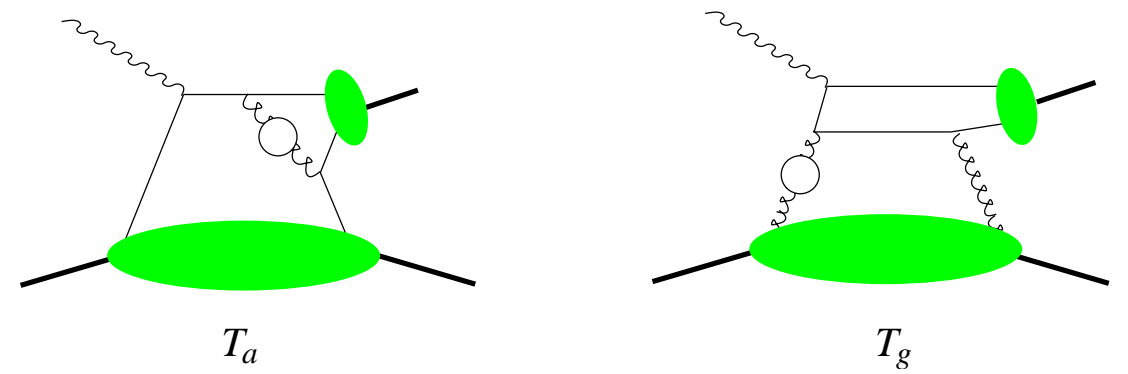

Figure 10: Example graphs giving rise to terms proportional to $\beta_{0}$ in the hard-scattering kernels $T_{a}$ and $T_{g}$. 

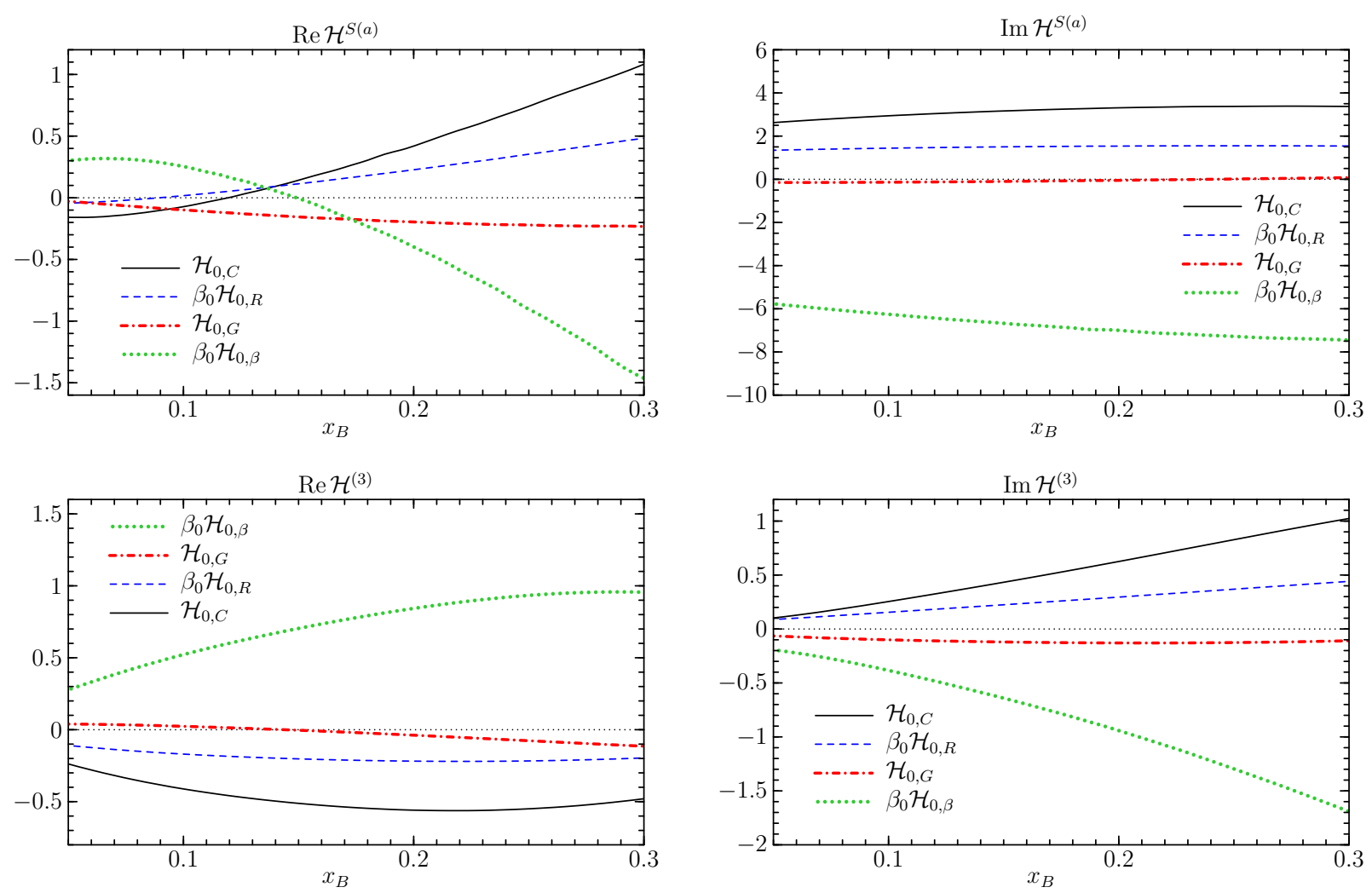

Figure 11: Individual terms (59) in the convolutions of the quark non-singlet kernel for $n=0$. The quark distributions are evaluated at $\mu_{G P D}=2 \mathrm{GeV}$ and the running coupling in the kernels at $\mu_{R}=2 \mathrm{GeV}$.

$\mathcal{H}_{0, C}$ has also the opposite sign compared to $\mathcal{H}_{0, \beta}$ but is similar in magnitude. We note that $\left|\mathcal{H}_{n, \beta}\right|$ increases with $n$, as can be seen from (86) and (88).

Let us briefly comment on the BLM scale setting prescription 23], which has been discussed in the context of exclusive meson production in [10, 24]. This prescription aims at including the corrections from graphs like those of Fig. 10 in the argument of the running coupling, and for the case at hand takes $\mu_{R}$ such that the contribution from $\mathcal{H}_{n, \beta}$ cancels against the one from $\mathcal{H}_{n, R} \ln \left(Q^{2} / \mu_{R}^{2}\right)$ in (59). As is evident from Fig. 11, this requires $\mu_{R}^{2}$ to be substantially lower than $Q^{2}$. For most of experimentally accessible kinematics, the resulting $\mu_{R}$ is in fact far below the region where perturbation theory can be applied. In such a situation, the perturbative running of $\alpha_{s}$ is often modified such that the coupling saturates for decreasing $\mu_{R}$. We note that in the context of our NLO analysis, the logarithm $\beta_{0} \ln \left(Q^{2} / \mu_{R}^{2}\right)$ in the hard-scattering kernel is intimately related with the perturbative running of $\alpha_{s}\left(\mu_{R}\right)$, so that keeping one while modifying the other is not obviously consistent.

We also remark that if $\mathcal{H}_{n, \beta}$ and $\mathcal{H}_{n, R} \ln \left(Q^{2} / \mu_{R}^{2}\right)$ are made to cancel by the BLM scale choice, one is left with a relatively large correction from $\mathcal{H}_{n, C}$. For scale choices where $\mu_{R}^{2}$ is closer to $Q^{2}$, one instead has a partial cancellation between $\mathcal{H}_{n, C}$ and $\mathcal{H}_{n, \beta}$. A more detailed analysis for the similar case of the electromagnetic form factor is given in [25], which also discusses the issue of Sudakov-type corrections we raised above.

Figure 12 shows the dependence of the convolutions on $\mu_{R}$ at fixed $\mu_{G P D}=Q$. Within the $\mu_{R}$ range shown we generally find a moderate scale dependence, both at LO and at NLO. An exception 

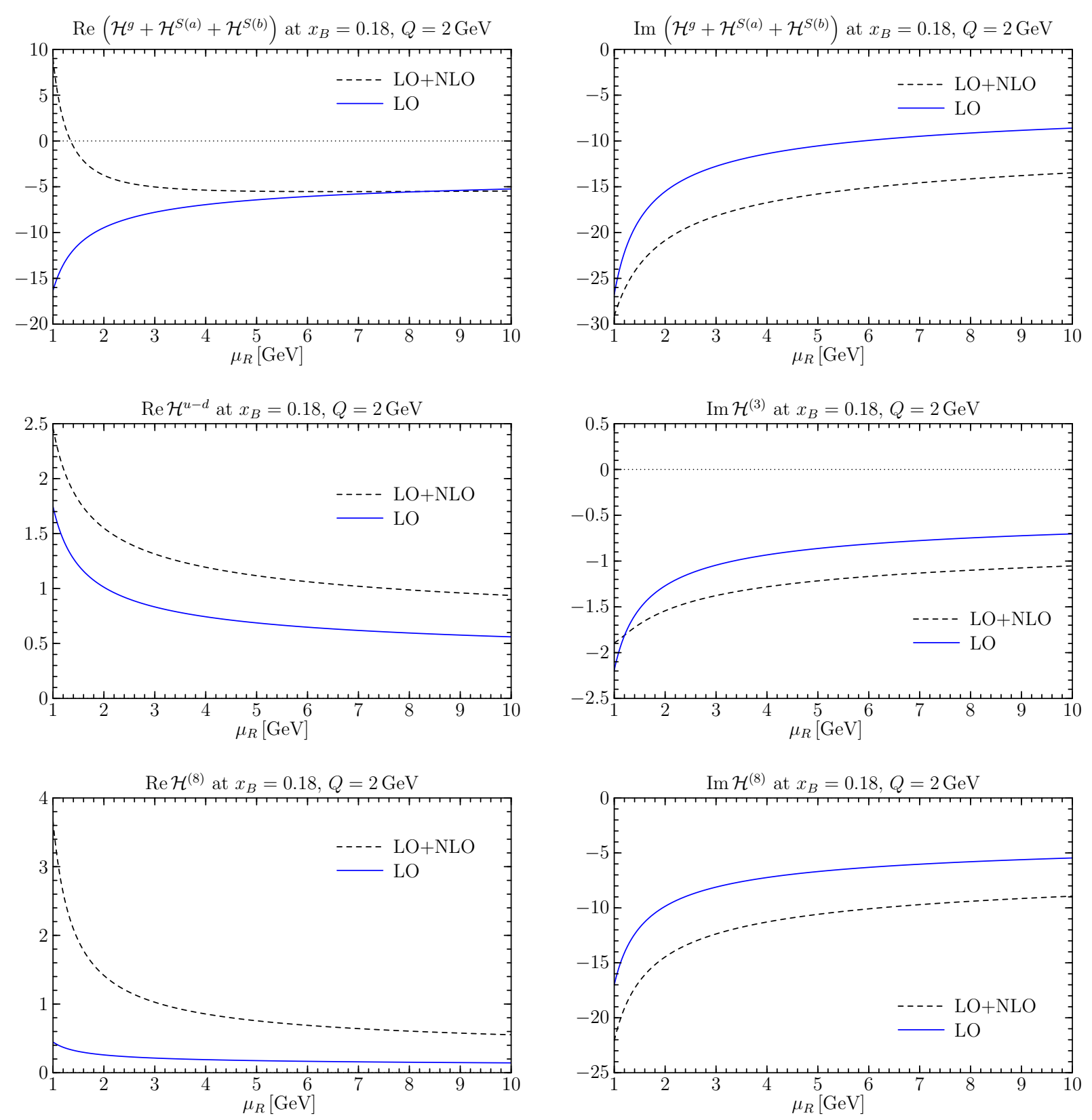

Figure 12: Dependence of the convolutions on $\mu_{R}$. The factorization scale is held fixed at $\mu_{G P D}=Q$. 

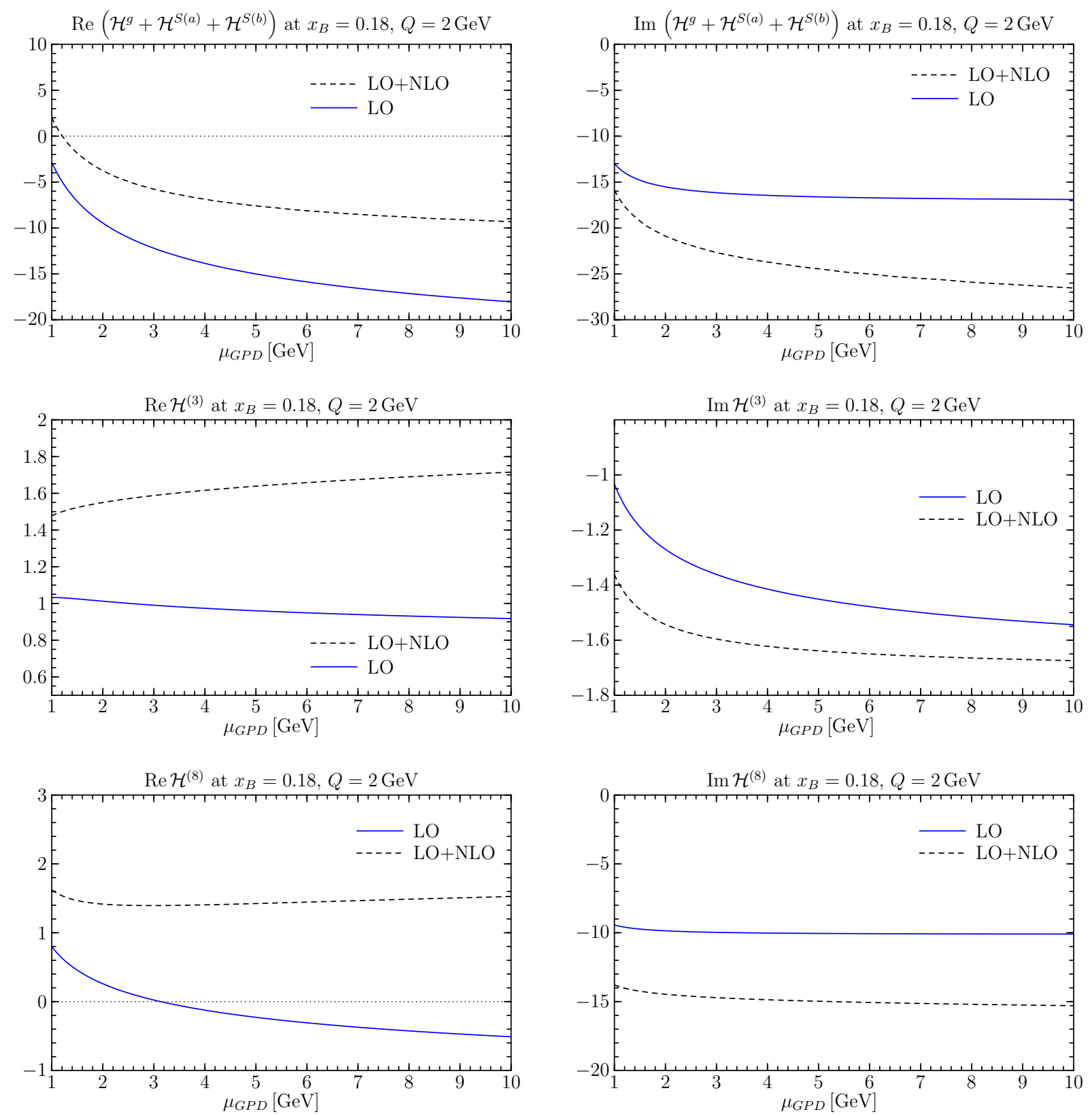

Figure 13: Dependence of the convolutions on $\mu_{G P D}$. The renormalization scale is held fixed at $\mu_{R}=Q$. 

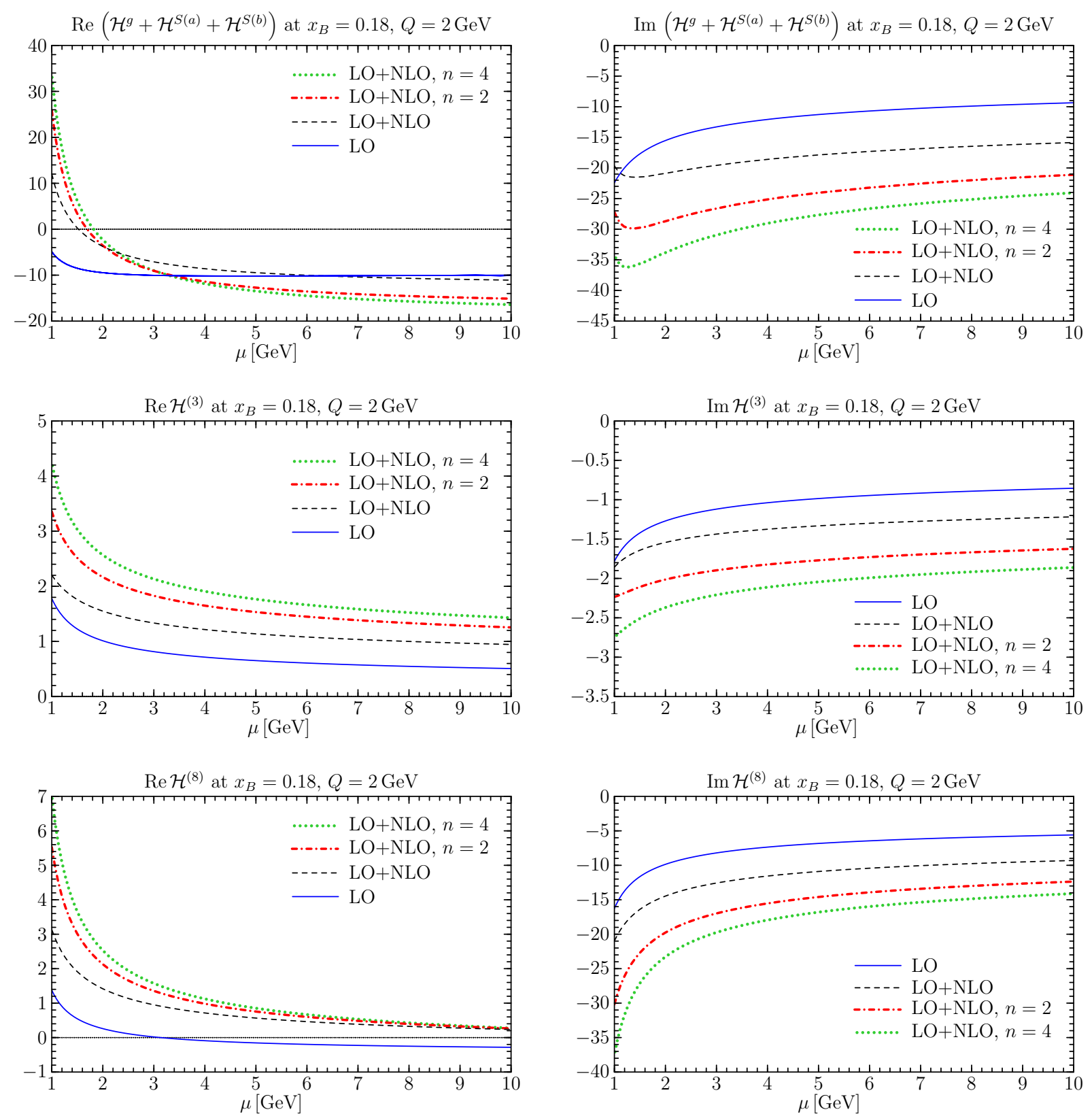

Figure 14: Dependence of the convolutions on the common scale $\mu=\mu_{R}=\mu_{G P D}=\mu_{D A}$. 

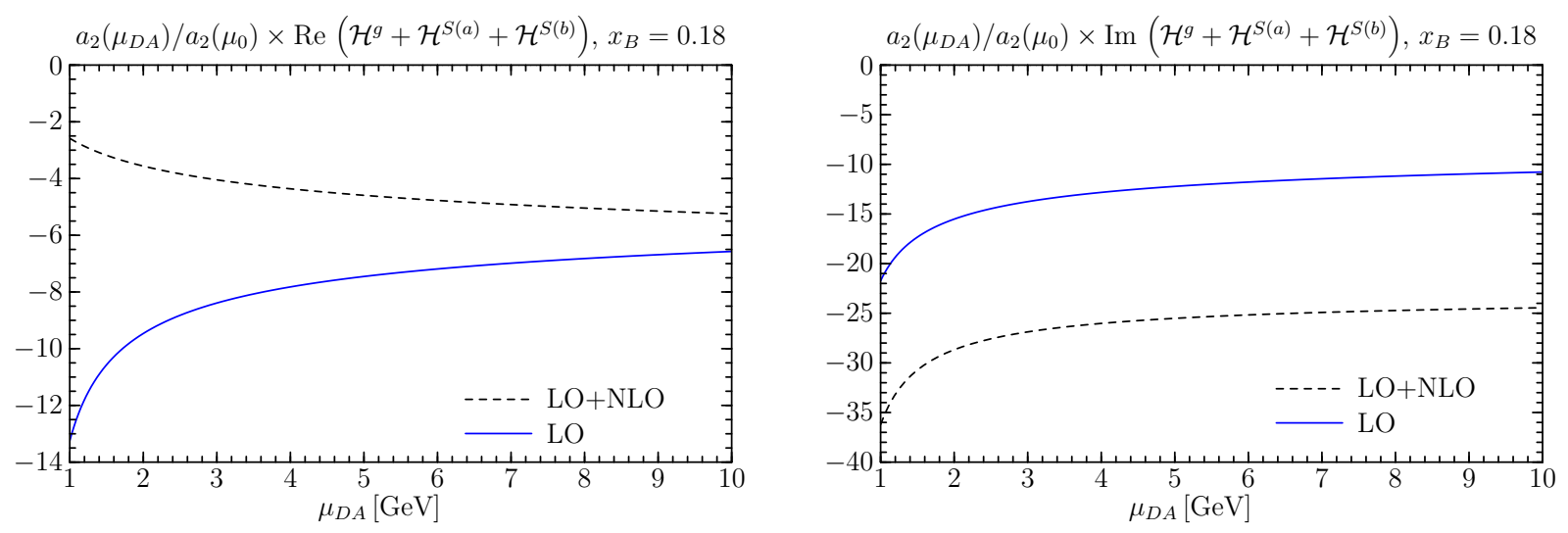

Figure 15: Dependence on the factorization scale $\mu_{D A}$ of the convolutions in the gluon and quark singlet sector multiplied by the scale dependence $a_{2}\left(\mu_{D A}\right) / a_{2}\left(\mu_{0}\right)$ of the corresponding Gegenbauer coefficient. The reference scale for $a_{2}$ is taken as $\mu_{0}=2 \mathrm{GeV}$, and the other scales are set to $\mu_{G P D}=\mu_{R}=Q=2 \mathrm{GeV}$.

is the region $\mu_{R} \lesssim 2 \mathrm{GeV}$, where the growth of the LO results simply reflects the growth of $\alpha_{s}\left(\mu_{R}\right)$. Note that with the parameters specified at beginning of Sect. 4 we have $\alpha_{s}^{(3)}(2 \mathrm{GeV})=0.30$ and $\alpha_{s}^{(3)}(1 \mathrm{GeV})=0.51$. The NLO results further contain explicit logarithms $\ln \left(Q^{2} / \mu_{R}^{2}\right)$, which in some cases can cause corrections to grow out of control, especially for the real parts of convolutions. We note that for $\operatorname{Re} \mathcal{H}^{(8)}$ the NLO correction is is unusually large compared with the LO term. This is because of a nearby zero in $x_{B}$, as is seen in Fig. 9, and should not be a reason of particular concern.

The variation of the convolutions with $\mu_{G P D}$ at fixed $\mu_{R}=Q$ is shown in Fig. 13, We again find a rather moderate scale dependence, except when $\mu_{G P D}$ becomes small. The dependence on a single scale $\mu=\mu_{R}=\mu_{G P D}$ is shown in Fig. 14. Note that in many cases the individual variation of $\mu_{R}$ decreases the amplitude in absolute size whereas the variation of $\mu_{G P D}$ increases it, with both tendencies partially canceling when the scales are set equal. Again we find that the scale dependence becomes quite drastic below $2 \mathrm{GeV}$.

We finally discuss the dependence on $\mu_{D A}$ for Gegenbauer indices $n>0$. According to (18) the convolutions $\mathcal{H}_{n}\left(\mu_{D A}\right)$ appear multiplied by $a_{n}\left(\mu_{D A}\right)$ in the process amplitude, where the scale dependence of both factors partially cancels. In Fig. 15] we therefore plot convolutions multiplied with $a_{2}\left(\mu_{D A}\right) / a_{2}\left(\mu_{0}\right)=\left[\alpha_{s}\left(\mu_{D A}\right) / \alpha_{s}\left(\mu_{0}\right)\right]^{\gamma_{2} / \beta_{0}}$ following the relation (16). The corresponding plots for $n=4$ and for convolutions in the quark non-singlet sector look very similar. We find that the dependence on $\mu_{D A}$ is slightly decreased when going to NLO.

\section{Proton helicity flip amplitudes}

We now turn to the convolutions of the hard-scattering kernels with the GPDs describing proton helicity flip. In this section we take $t=-0.4 \mathrm{GeV}^{2}$, which is the value for which will present estimates for observables in the next section.

In Figs. 16 and 17 we compare the LO and NLO terms of the convolutions in the gluon and quark singlet sector for the two models described in Sect. 3.1. For model 1 the individual corrections for gluon and quark convolutions look quite similar to those we saw for $\mathcal{H}$ in the previous section. The sum of gluon and quark singlet contributions at LO is however very small in this model because of cancellations, so that the NLO term dominates in a wide kinematical region. In model 2 the 

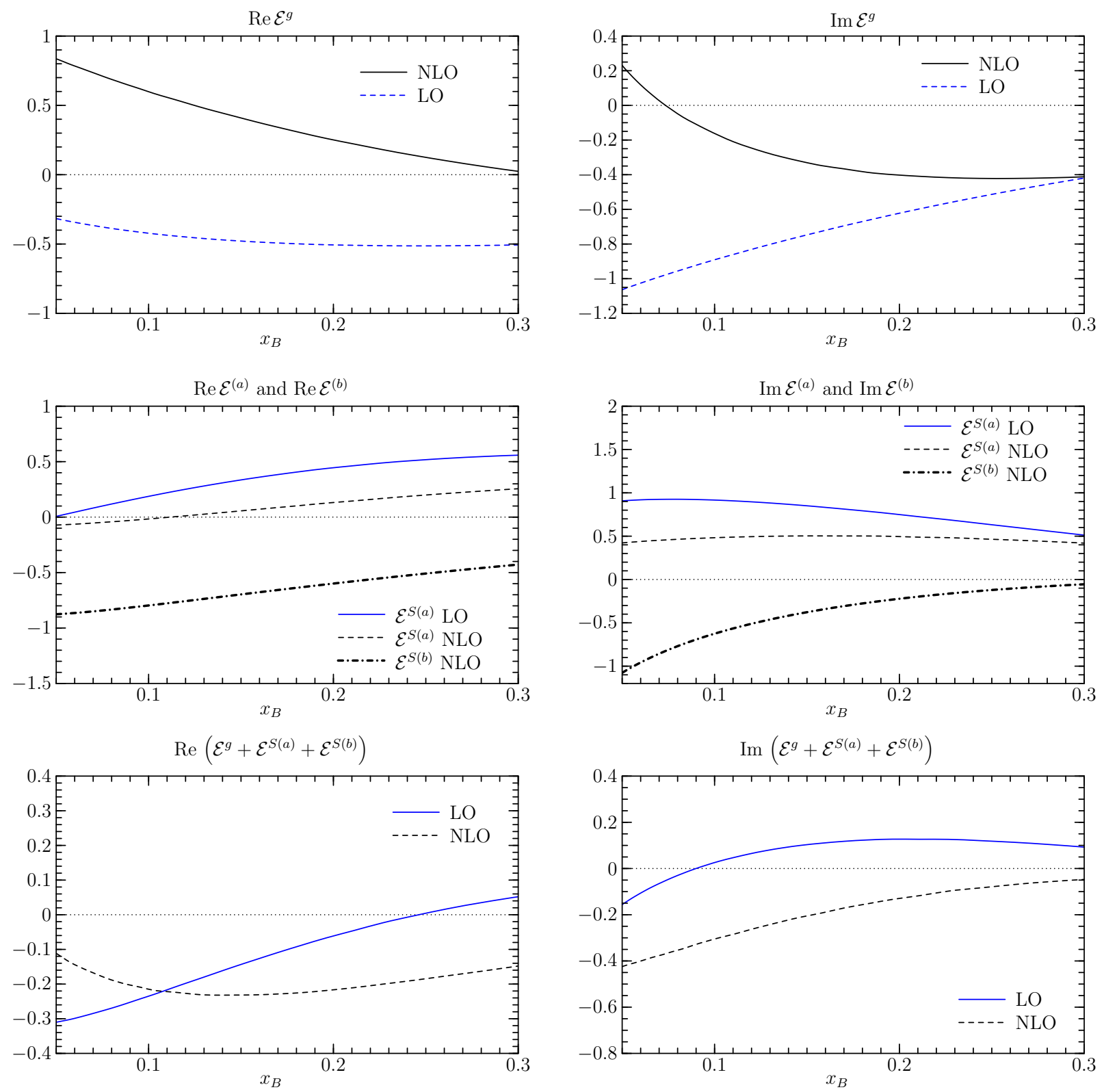

Figure 16: LO and NLO terms of the convolutions in the gluon and quark singlet sector for model 1 at $Q=2 \mathrm{GeV}$ and $t=-0.4 \mathrm{GeV}^{2}$. The scales are set to $\mu_{R}=\mu_{G P D}=Q$. 

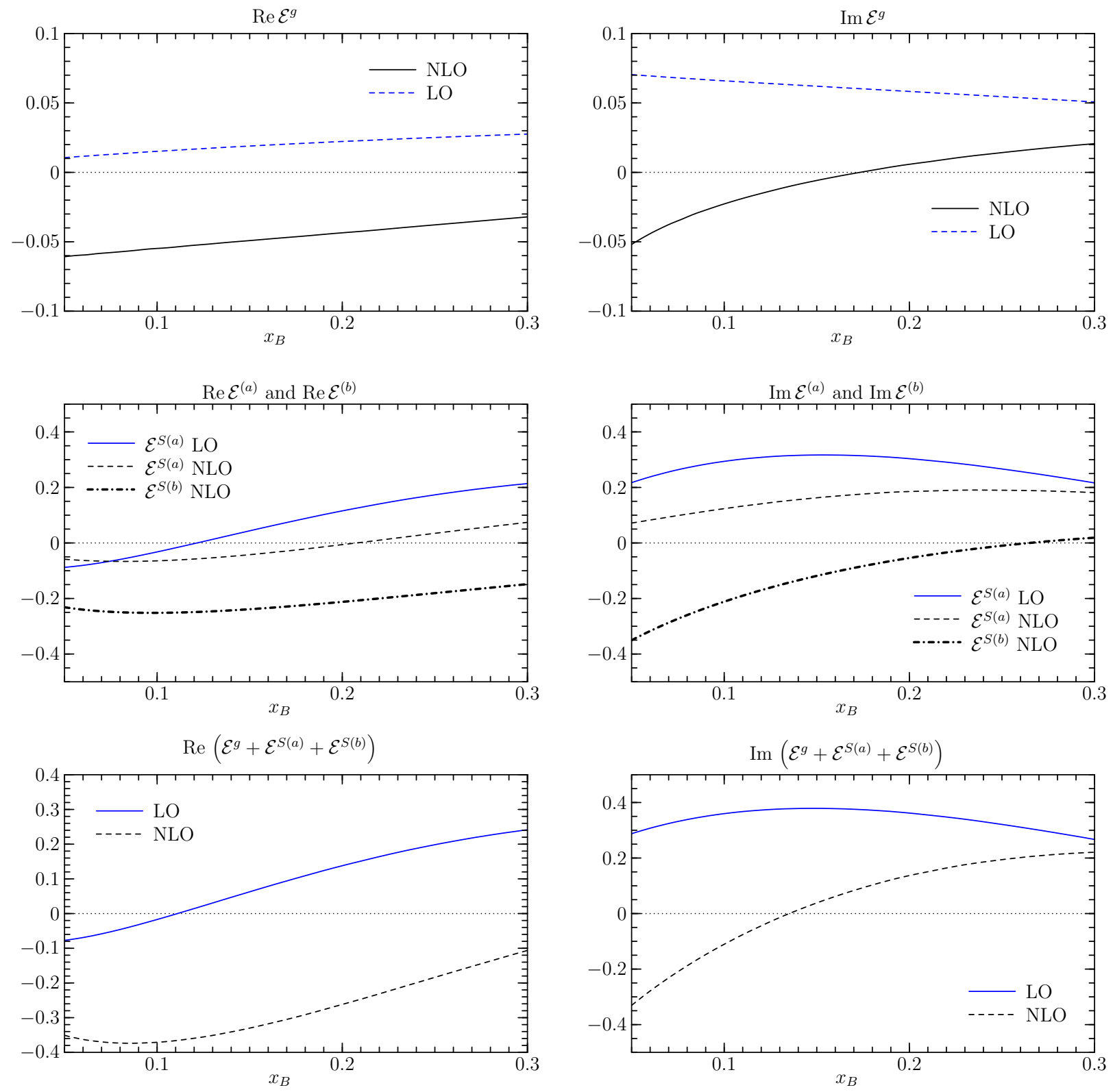

Figure 17: As Fig. 16 but for model 2. 

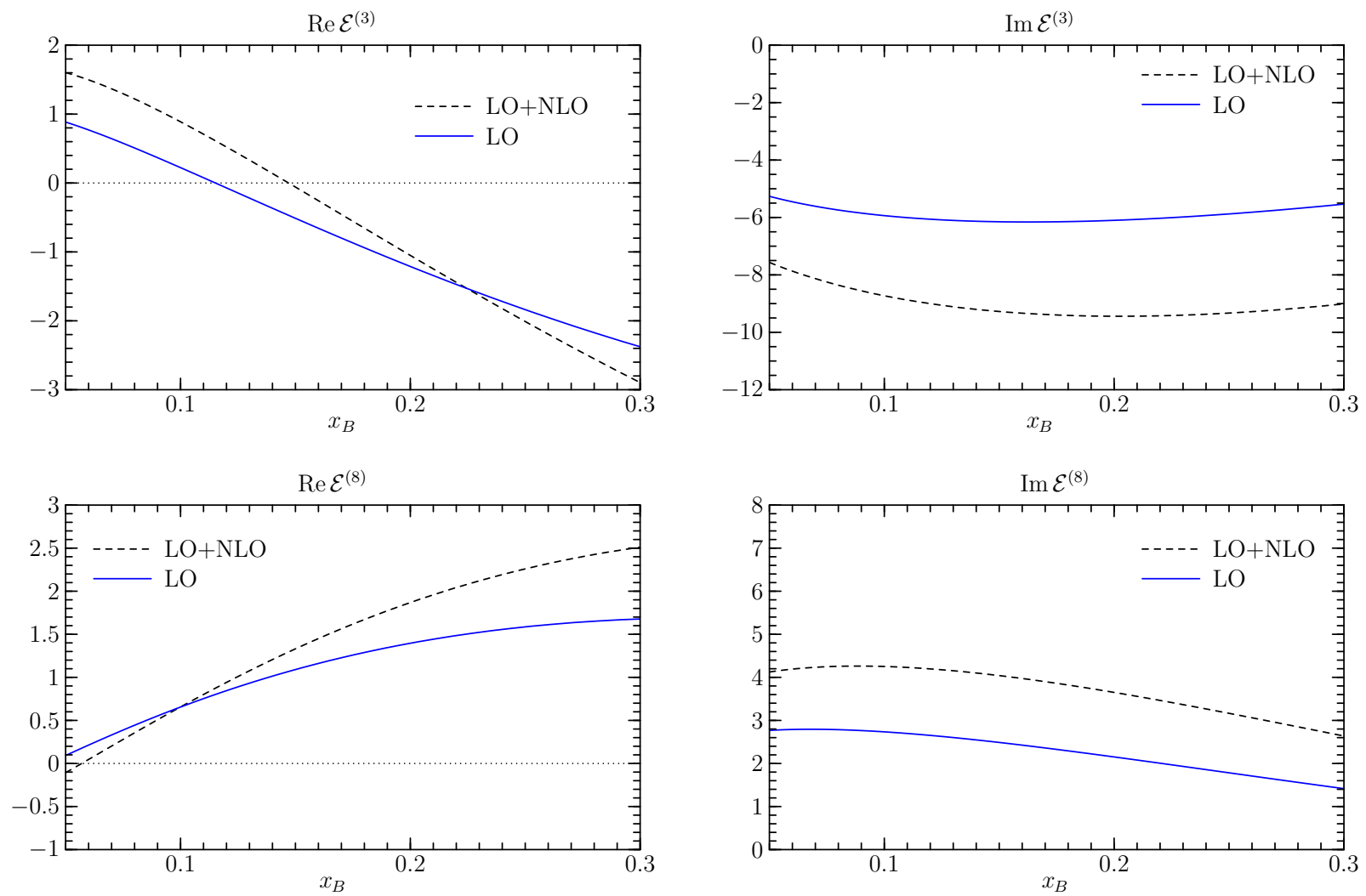

Figure 18: LO and NLO terms of the convolutions in the quark non-singlet sector for model 1 at $Q=2 \mathrm{GeV}$ and $t=-0.4 \mathrm{GeV}^{2}$, with $\mu_{R}=\mu_{G P D}=Q$. 

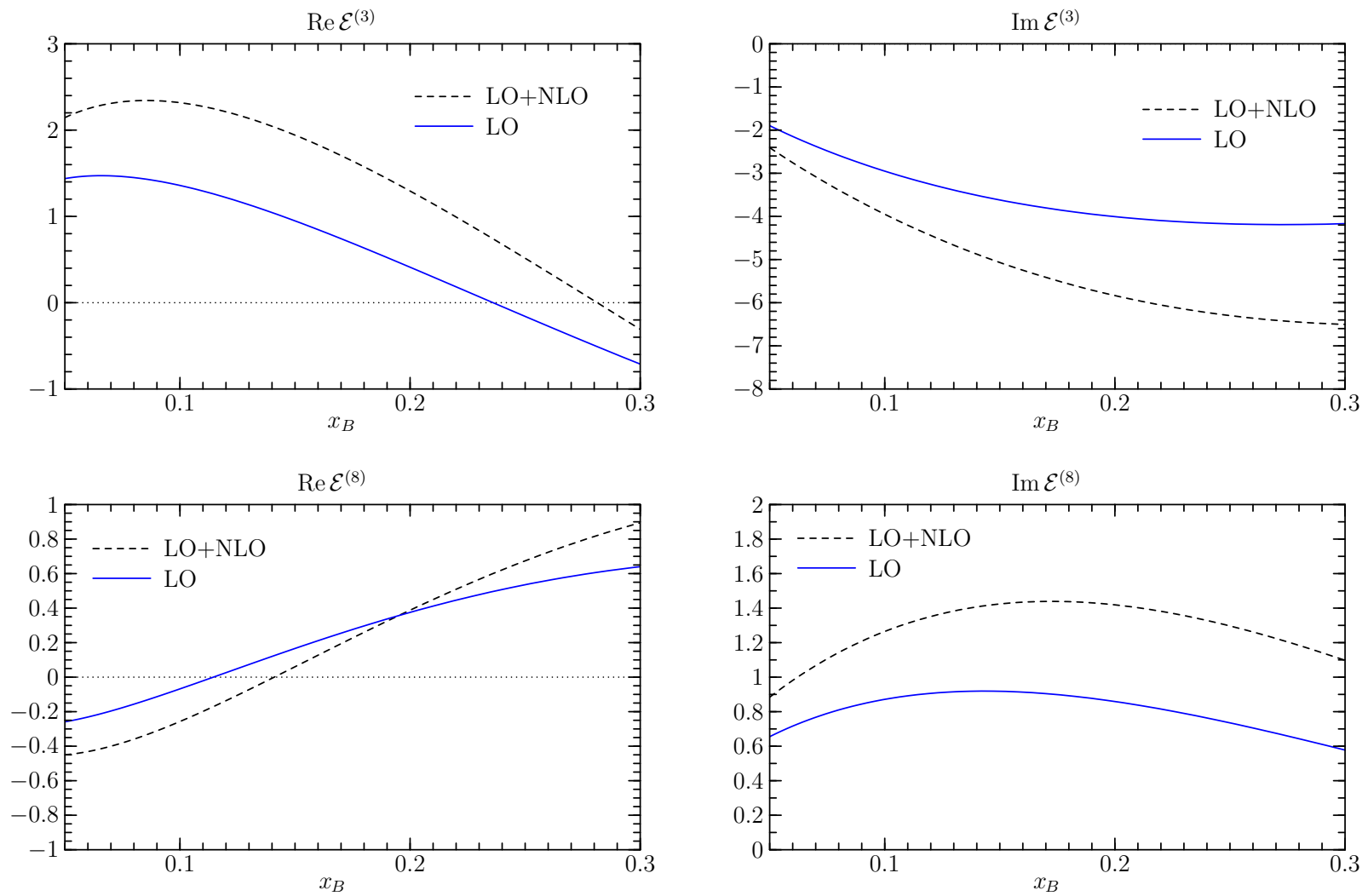

Figure 19: As Fig. 18 but for model 2. 
gluon contributions are nearly absent, so that the quark singlet contribution dominates in this sector. We note that, contrary to the individual terms, the sum of gluon and quark singlet contributions comes out to be rather similar in the two models and is small compared with the flavor non-singlet contributions shown in Figs. 18 and 19, According to our discussion at the end of Sect. 3.1 this has its origin in the sum rule (43) for the second moment of $E$ at $t=0$, so that we expect a small net contribution from gluons and the quark singlet in large class of models for $E$.

As shown in Figs. 18 and 19, the NLO corrections to the quark non-singlet convolutions are relatively moderate but not small, similarly to the case of $\mathcal{H}$. The size of the convolutions is quite different in the two models, indicating the important role played at intermediate $x_{B}$ by sea quarks in model 1. Let us recall that with the double distribution ansatz (33) the GPDs at $x \sim \xi$ are sensitive to forward parton distributions with momentum fractions well below $\xi$, as discussed in Sect. 4.3.3 of [17].

\section{Cross sections and asymmetries}

Having studied in detail the building blocks of the scattering amplitude for vector meson production, we now combine them to observables. We recall that to leading order in $1 / Q$ there are just two of these: the unpolarized $\gamma^{*} p$ cross section and the asymmetry for a transversely polarized target, both referring to longitudinal polarization of virtual photon and produced meson. The ep cross section in the leading $1 / Q$ approximation can be written as

$$
\frac{d \sigma(e p \rightarrow e p V)}{d t d Q^{2} d y d \phi d \phi_{S}}=\frac{\alpha}{4 \pi^{3}} \frac{1-x_{B}}{Q^{2}} \frac{1-y}{y} \frac{d \sigma_{L}}{d t}\left[1+S_{T} \sin \left(\phi-\phi_{S}\right) A_{U T}\right]
$$

where $y$ is the usual inelasticity variable for deep inelastic scattering and $S_{T}$ denotes the transverse component of the target polarization. $\phi$ is the azimuthal angle between lepton plane and hadron plane, and $\phi_{S}$ is the azimuthal angle between lepton plane and target spin vector, both defined according to the Trento convention [40]. The $\gamma^{*} p$ cross section $d \sigma_{L} / d t$ and the polarization asymmetry $A_{U T}$ depend on $x_{B}, Q^{2}$ and $t$. To leading order in $1 / Q$ they are given by

$$
\frac{d \sigma_{L}}{d t}=\frac{\pi^{2}}{9} \frac{\alpha}{Q^{6}} \frac{\left(2-x_{B}\right)^{2}}{1-x_{B}} f_{V}^{2}\left[\left(1-\xi^{2}\right)\left|\mathcal{H}_{V}\right|^{2}-\left(t /\left(4 m_{p}^{2}\right)+\xi^{2}\right)\left|\mathcal{E}_{V}\right|^{2}-2 \xi^{2} \operatorname{Re}\left(\mathcal{E}_{V}^{*} \mathcal{H}_{V}\right)\right]
$$

and

$$
A_{U T}=\frac{\sqrt{t_{0}-t}}{m_{p}} \frac{\sqrt{1-\xi^{2}} \operatorname{Im}\left(\mathcal{E}_{V}^{*} \mathcal{H}_{V}\right)}{\left(1-\xi^{2}\right)\left|\mathcal{H}_{V}\right|^{2}-\left(t /\left(4 m_{p}^{2}\right)+\xi^{2}\right)\left|\mathcal{E}_{V}\right|^{2}-2 \xi^{2} \operatorname{Re}\left(\mathcal{E}_{V}^{*} \mathcal{H}_{V}\right)},
$$

where $t_{0}=-4 m_{p}^{2} \xi^{2} /\left(1-\xi^{2}\right)$. Here we have combined the convolutions (19) into

$$
\mathcal{F}_{V}=Q_{V} \sum_{n=0}^{\infty} a_{n}\left[\mathcal{F}_{n}^{g}+\mathcal{H}_{n}^{S(a)}+\mathcal{F}_{n}^{S(b)}+e_{V}^{(3)} \mathcal{F}_{n}^{(3)}+e_{V}^{(8)} \mathcal{F}_{n}^{(8)}\right],
$$

with analogous combinations for $\mathcal{H}_{V}$ and $\mathcal{E}_{V}$. In the remainder of this section we take the asymptotic form of the meson distribution amplitude, i.e. we set $a_{n}=0$ for $n \geq 2$. As long as $\mathcal{E}_{V}$ is not much larger than $\mathcal{H}_{V}$, the cross section (61) is dominated by the term with $\left|\mathcal{H}_{V}\right|^{2}$ in a wide range of kinematics, where the prefactors $\xi^{2}$ and $t /\left(4 m_{p}^{2}\right)$ of the other terms are small. The asymmetry (62) is then approximately given by

$$
A_{U T} \approx \frac{\sqrt{t_{0}-t}}{m_{p}} \frac{\operatorname{Im}\left(\mathcal{E}_{V}^{*} \mathcal{H}_{V}\right)}{\left|\mathcal{H}_{V}\right|^{2}}=\frac{\sqrt{t_{0}-t}}{m_{p}}\left|\frac{\mathcal{E}_{V}}{\mathcal{H}_{V}}\right| \sin \delta_{V},
$$



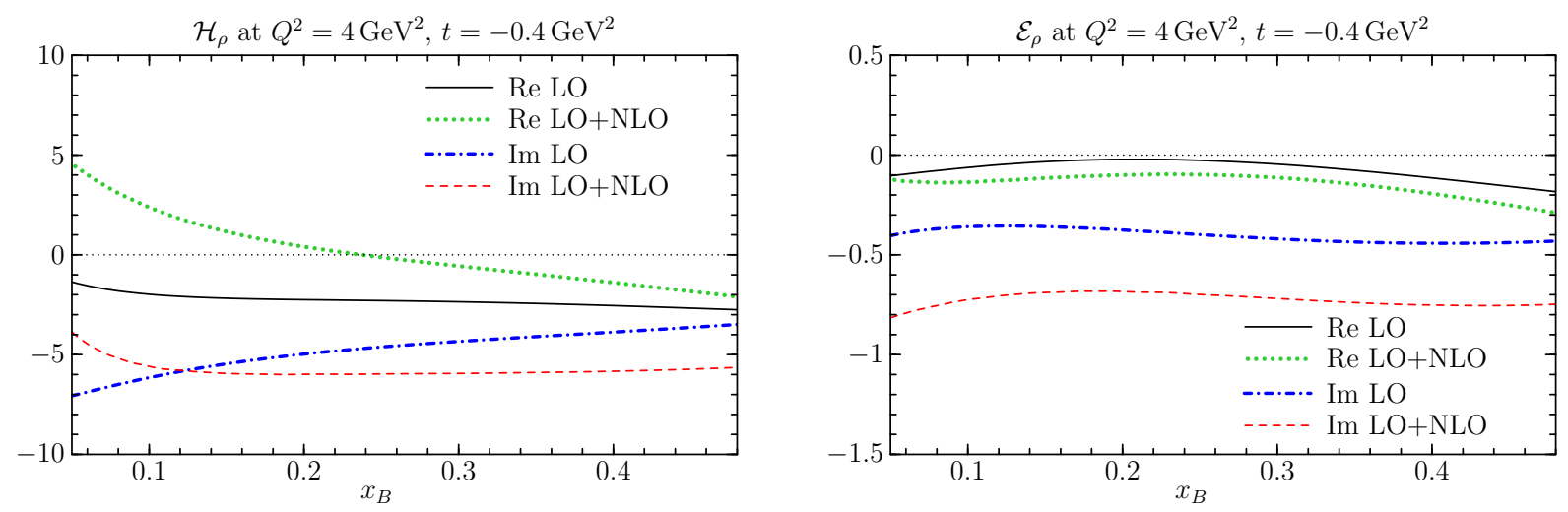

Figure 20: Real and imaginary parts of the convolutions (63) for $\rho$ production at $Q=2 \mathrm{GeV}$ and $t=-0.4 \mathrm{GeV}^{2}$, with model 1 taken for the proton helicity-flip distributions. The scales are set to $\mu_{R}=\mu_{G P D}=Q$.
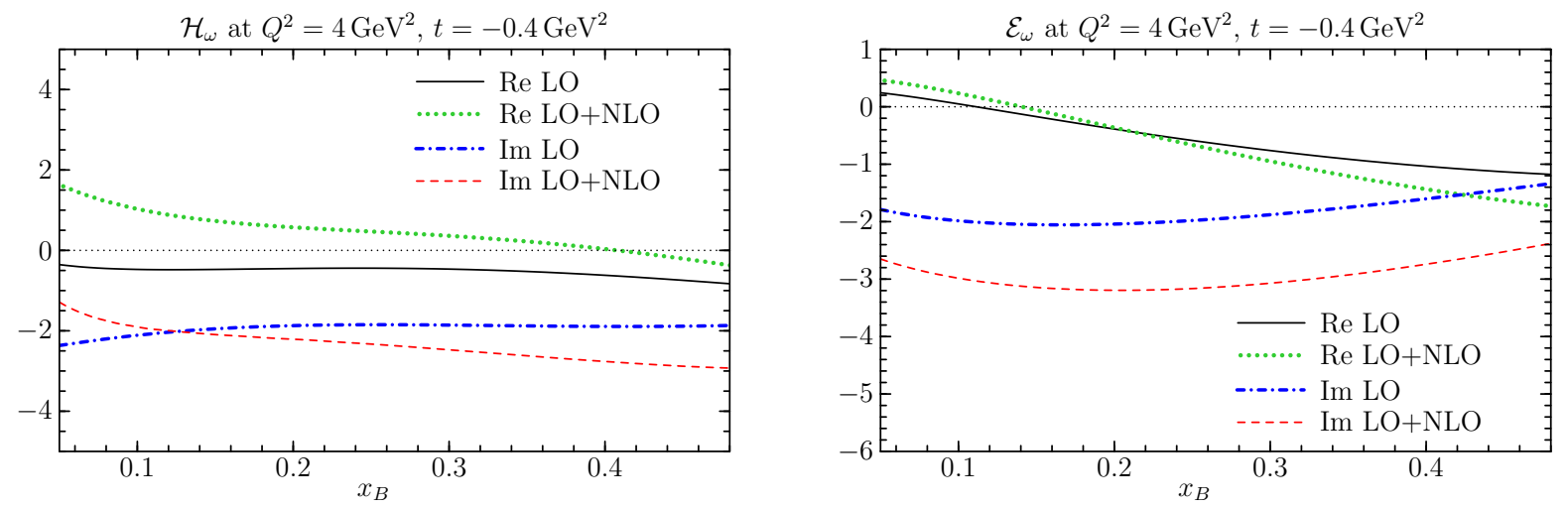

Figure 21: As Fig. 20 but for $\omega$ production.

where $\delta_{V}=\arg \left(\mathcal{H}_{V} / \mathcal{E}_{V}\right)$ is the relative phase between $\mathcal{H}_{V}$ and $\mathcal{E}_{V}$.

Figure 20 shows the real and imaginary parts of the convolutions appearing in (61) and (62). $\mathcal{H}_{\rho}$ is dominated by the gluon and quark singlet part, and in line with our discussion in Sect. 5 we find rather moderate corrections for the imaginary part but a very unstable real part in a wide range of $x_{B}$. As for $\mathcal{E}_{\rho}$, its real part is very small and subject to large relative corrections, whereas its imaginary part is much larger and receives corrections of order $100 \%$. As we see in Fig. 18, the individual flavor non-singlet combinations $\mathcal{E}^{(3)}$ and $\mathcal{E}^{(8)}$ are less affected by corrections, but they have opposite sign and partially cancel in the sum relevant for $\rho$ production. The rather small but unstable contribution from the gluon and quark singlet terms is hence important in this channel and largely responsible for the NLO corrections seen in Fig. 20. As a further consequence of the cancellations just mentioned, the size of $\mathcal{E}_{\rho}$ is tiny compared with $\mathcal{H}_{\rho}$. The quark flavor combination relevant for $\rho$ production is $2 u+d$, where in our model the flavor combinations add for $H$ but largely cancel for $E$.

For $\omega$ production we see in Fig. 21 that at smaller $x_{B}$ the convolution $\mathcal{H}_{\omega}$ is about $1 / 3$ of $\mathcal{H}_{\rho}$, which follows from the dominance of the gluon contribution as seen in (6) and (7), whereas at larger $x_{B}$ differences between the two channels appear. $\mathcal{E}_{\omega}$ is much bigger than $\mathcal{E}_{\rho}$ since in the combination $2 u-d$ the contributions of $u$ and $d$ quarks add for $E$, and the size of its radiative corrections reflects 
the one of $\mathcal{E}^{(3)}$ in Fig. 18. We note that the dominance of the imaginary over the real part in $\mathcal{E}_{\omega}$ and $\mathcal{E}_{\rho}$ is less pronounced in model 2, as can be anticipated by comparing Figs. 18 and 19.

The cross section $d \sigma_{L} / d t$ for $\rho$ production is dominated by $\left(\operatorname{Im} \mathcal{H}_{\rho}\right)^{2}$, except for contributions from $\left(\operatorname{Re} \mathcal{H}_{\rho}\right)^{2}$ at small $x_{B}$ for NLO and at large $x_{B}$ for LO. Given the size of corrections to $\operatorname{Im} \mathcal{H}_{\rho}$ in Fig. 20] we thus have quite substantial NLO effects in the cross section at $Q^{2}=4 \mathrm{GeV}^{2}$, as shown in Fig. 22. For $Q^{2}=9 \mathrm{GeV}^{2}$ and $x_{B}>0.1$ the relative corrections decrease. The plot has been calculated with model 1 for $E$, but since its contribution to $d \sigma_{L} / d t$ is negligible the corresponding curves for model 2 look very similar. To obtain an estimate of scale uncertainties, we show bands corresponding to $\mu=\mu_{R}=\mu_{G P D}$ between $2 \mathrm{GeV}$ and $2 Q$. Given our discussion in the previous section, we do not consider it meaningful to go to scales below $2 \mathrm{GeV}$, so that the bands in the figure are strongly asymmetric. For $Q^{2}=4 \mathrm{GeV}^{2}$ they go only in one direction, and the band of the LO result does not provide an estimate for the size of the NLO corrections, which turn out to go in the other direction.

We have a very peculiar situation for the polarization asymmetry $A_{U T}$ in $\rho$ production, which as shown in Figs. 23 and 24 is very small in both models 1 and 2 due to the cancellations in $\mathcal{E}_{\rho}$ discussed above. $A_{U T}$ changes quite dramatically from LO to NLO in a wide range of kinematics, clearly because of the NLO corrections in the numerator. A closer look at Fig. 20 reveals that the large perturbative corrections in $\operatorname{Im}\left(\mathcal{E}_{\rho}^{*} \mathcal{H}_{\rho}\right)$ are mainly due to the large corrections to both $\operatorname{Re} \mathcal{H}_{\rho}$ and $\operatorname{Re} \mathcal{E}_{\rho}$. These hardly affect the unpolarized cross section, which is strongly dominated by $\operatorname{Im} \mathcal{H}_{\rho}$. At higher $Q^{2}$ the instability of $A_{U T}$ is less pronounced, and in model 2 we even have quite small corrections. We note that the bands from the scale variation at LO order are extremely narrow in Figs. 23 and 24. This is because the scale variation of $\alpha_{s}\left(\mu_{R}\right)$ cancels in the ratio $A_{U T}$ at LO and because in the kinematics we are looking at, the $\mu_{G P D}$ dependence of $\mathcal{H}_{\rho}$ and $\mathcal{E}_{\rho}$ is rather weak. In this situation, the scale uncertainty of the LO result does obviously not provide a good estimate for the size of higher-order corrections. Let us finally remark that at $t=-0.4 \mathrm{GeV}^{2}$ the asymmetry $A_{U T}$ must go to zero as $x_{B}$ tends to 0.484 because of the prefactor $\sqrt{t_{0}-t}$ in (62).

The cross section for $\omega$ production is shown in Fig. 25] and shows a similar pattern of NLO corrections to the one in $\rho$ production, reflecting the similar pattern of corrections we have seen for $\operatorname{Im} \mathcal{H}_{\rho}$ and $\operatorname{Im} \mathcal{H}_{\omega}$. As a result the ratio of cross sections $d \sigma_{L} / d t$ in the two channels is quite stable under radiative corrections, as seen in Fig. 26. The target polarization asymmetry, shown in Fig. 27 for model 1, changes however drastically between LO and NLO at small to intermediate $x_{B}$. This is because $\operatorname{Im} \mathcal{E}_{\omega}$ then dominates over $\operatorname{Re} \mathcal{E}_{\omega}$, so that its product with the unstable convolution $\operatorname{Re} \mathcal{H}_{\omega}$ controls the numerator of the asymmetry. The absolute size of $A_{U T}$ can be large in this channel since $\left|\mathcal{E}_{\omega}\right| \sim\left|\mathcal{H}_{\omega}\right|$ in our model. According to Fig. 21, the relative phase $\delta_{\omega}$ is close to zero at LO for $x_{B} \lesssim 0.3$, so that the factor $\sin \delta_{\omega}$ in (64) makes $A_{U T}$ small and prone to large radiative corrections.

Let us finally take a look at $\phi$ production. At LO this channel is strongly dominated by gluon exchange, since in our models strange quark distributions are small for $H$ and even more so for $E$. At NLO we have further contributions from the pure singlet terms $\mathcal{H}^{S(b)}$ and $\mathcal{E}^{S(b)}$, which are not negligible. We see in Fig. 28 that the NLO corrections to the cross section are large at small $x_{B}$ and slowly decrease with $x_{B}$. Except for the region of small $x_{B}$, this pattern is quite different from the one in $\rho$ production, so that the cross section ratio for the two channels receives important corrections at larger $x_{B}$ as we see in Fig. 29, The asymmetry $A_{U T}$ is essentially zero at LO, because in our model the relative phase $\delta_{\phi}$ between $\mathcal{H}_{\phi}$ and $\mathcal{E}_{\phi}$ is very close to zero. This changes at NLO, where in model 1 we obtain a small to moderate $A_{U T}$, as shown in Fig. 30. 

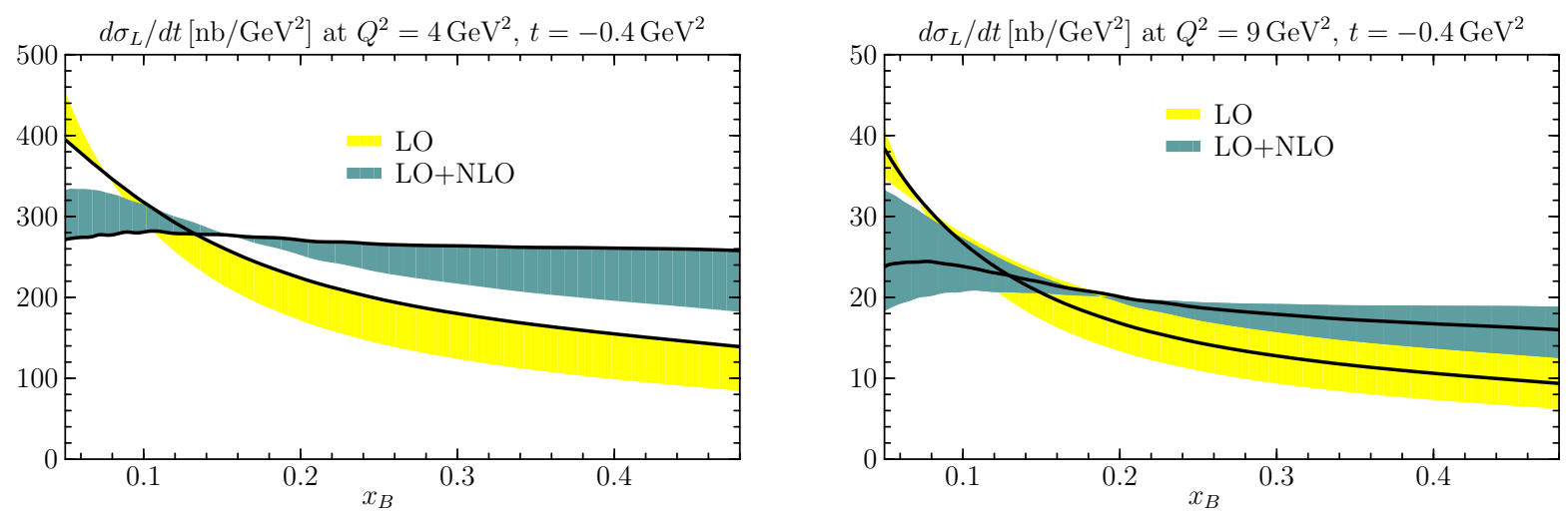

Figure 22: Longitudinal cross section for $\gamma^{*} p \rightarrow \rho p$ in model 1. Bands correspond to the range $2 \mathrm{GeV}<\mu<4 \mathrm{GeV}$ in the left and to $2 \mathrm{GeV}<\mu<6 \mathrm{GeV}$ in the right plot, and solid lines to $\mu=Q$ in both cases.
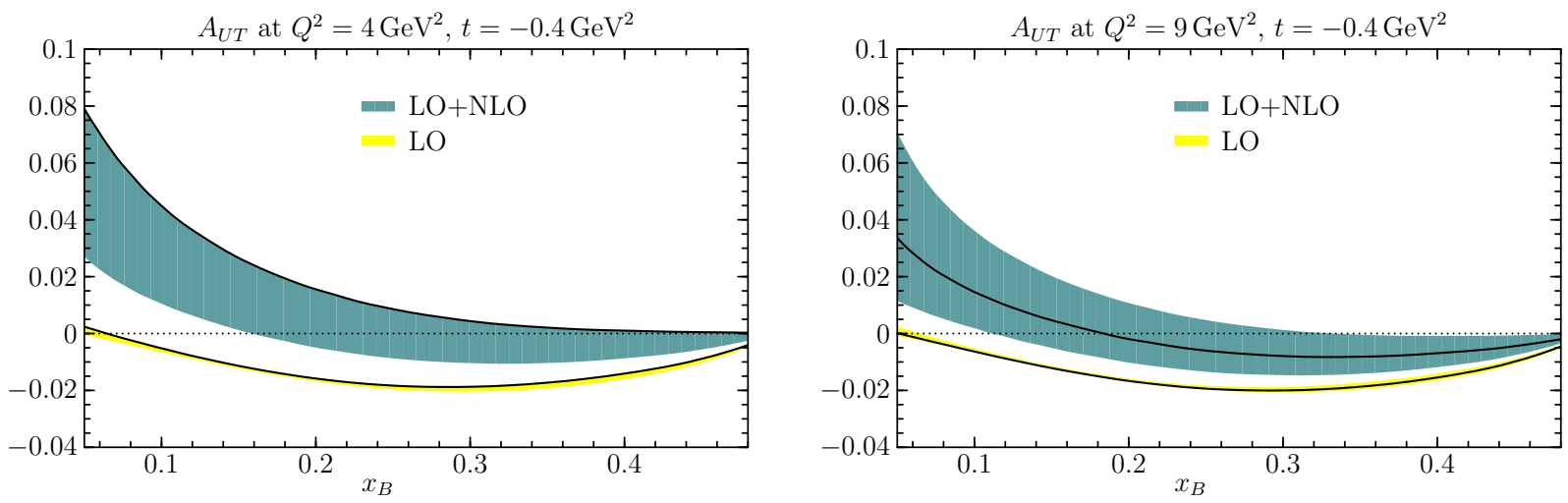

Figure 23: The transverse target spin asymmetry defined in (60), calculated for model 1. The meaning of the bands and solid lines is as in Fig. 22.
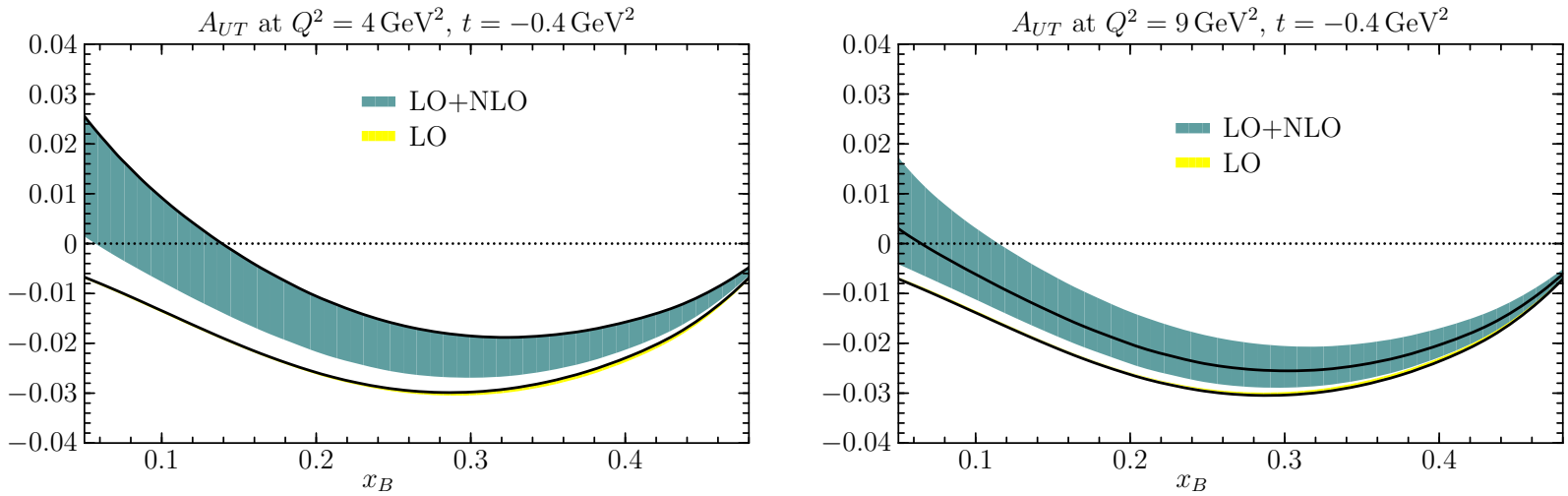

Figure 24: As Fig. 23 but for model 2. 

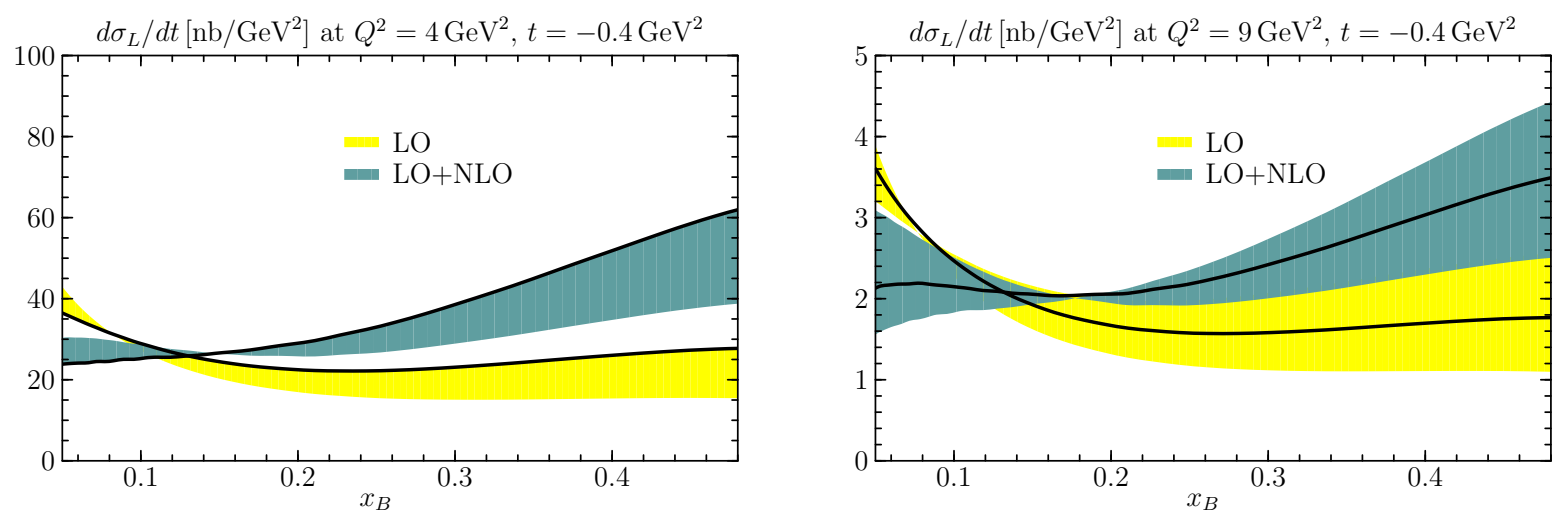

Figure 25: As Fig. 22 but for $\gamma^{*} p \rightarrow \omega p$.
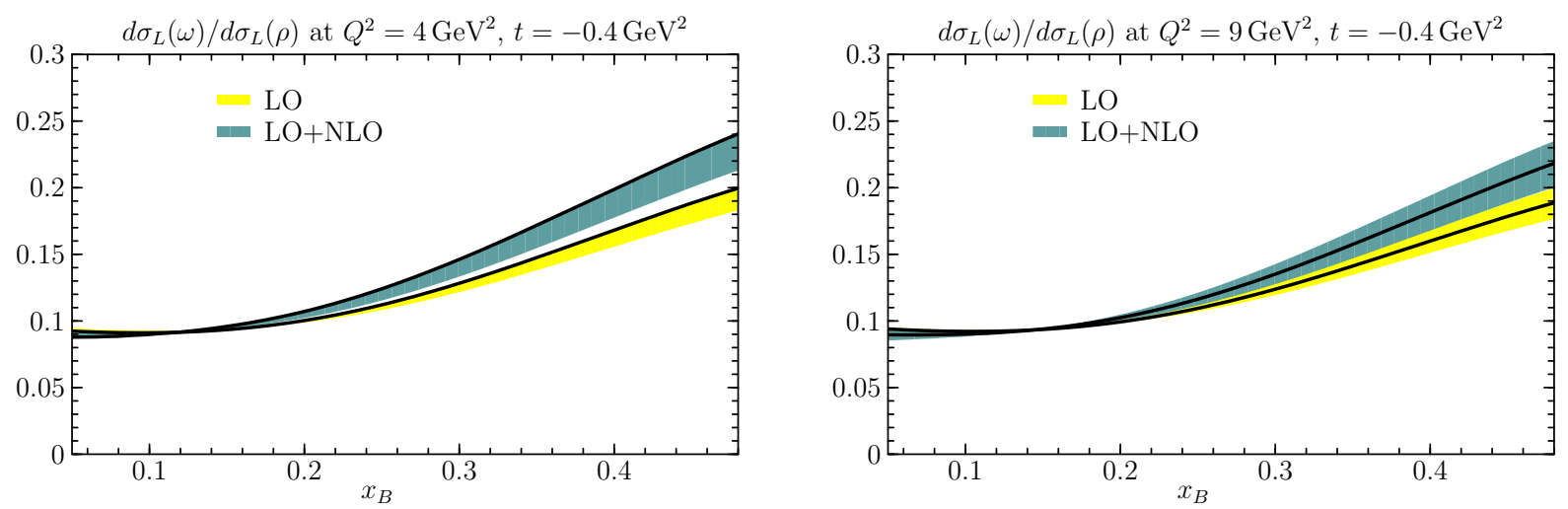

Figure 26: Ratio of cross sections $d \sigma_{L} / d t$ for $\omega$ and $\rho$ production in model 1 . The meaning of the bands and solid lines is as in Fig. 22 .
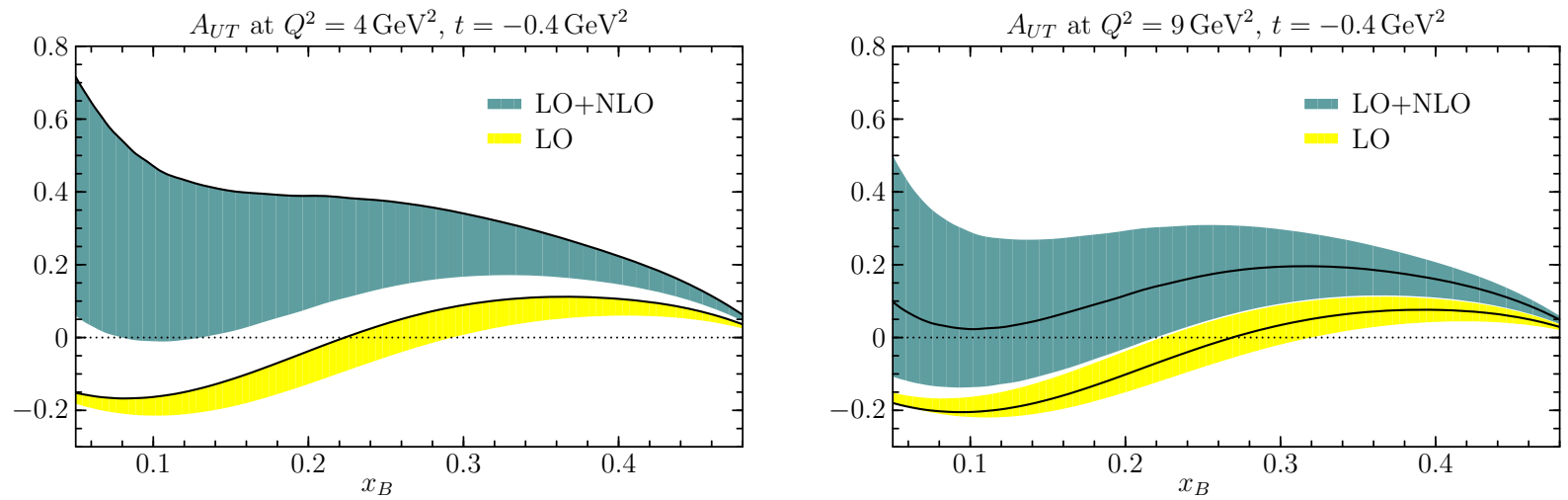

Figure 27: As Fig. 23 but for $\gamma^{*} p \rightarrow \omega p$. 

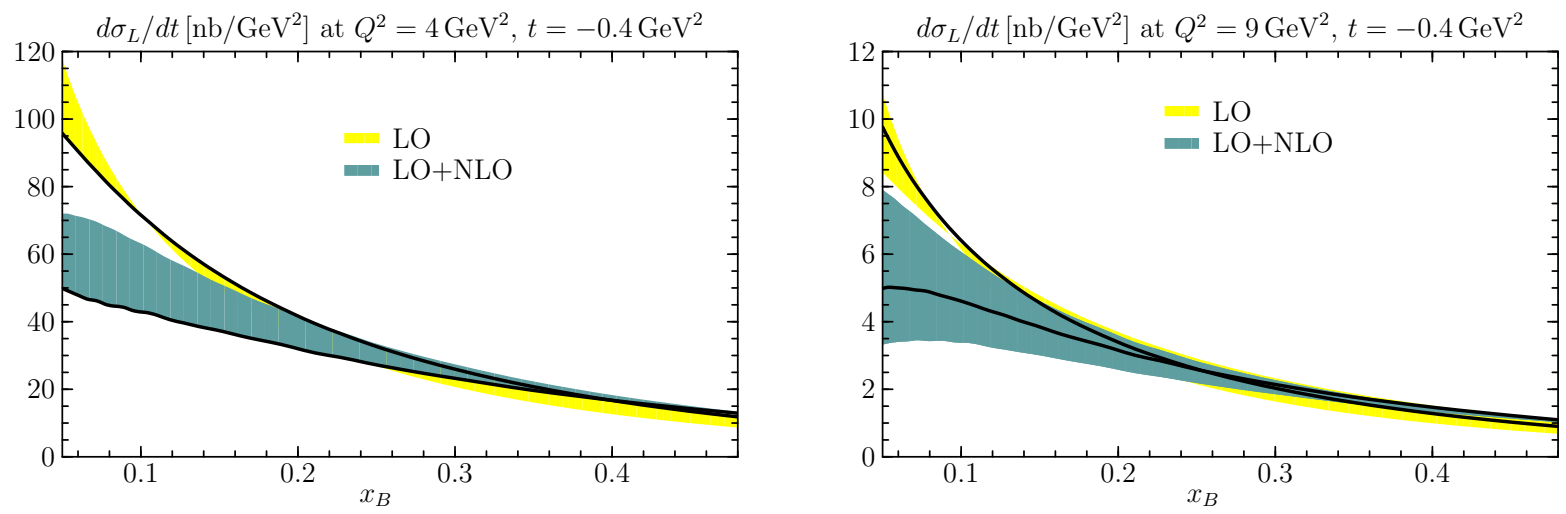

Figure 28: As Fig. 22 but for $\gamma^{*} p \rightarrow \phi p$.
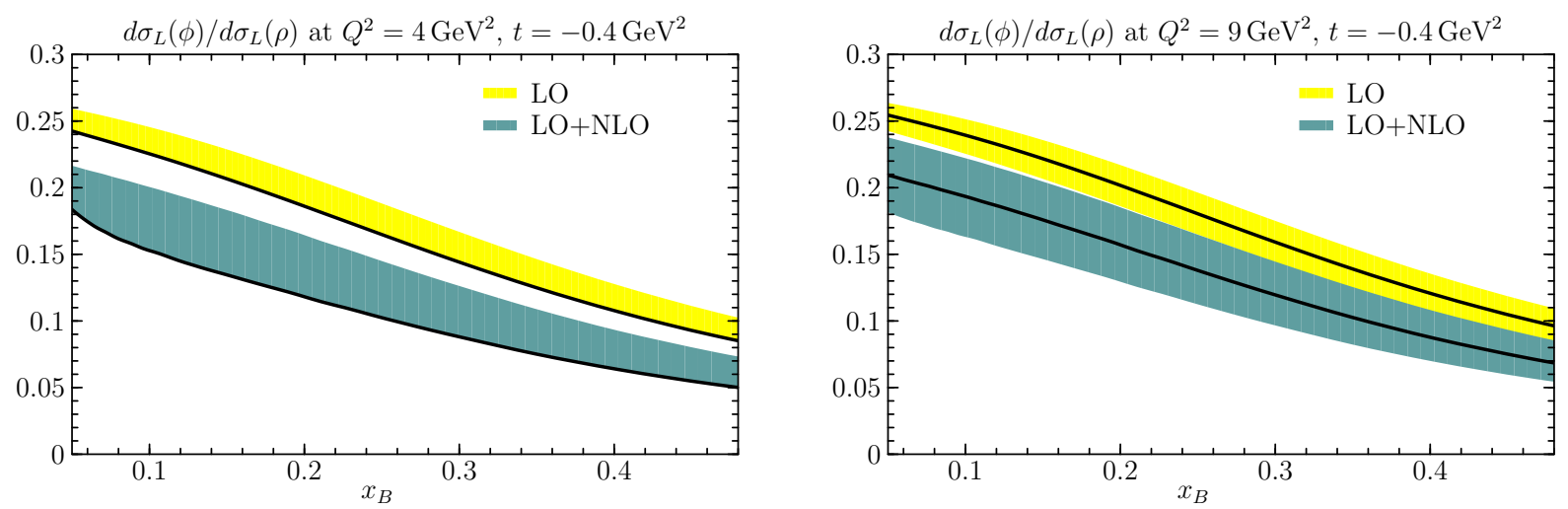

Figure 29: Ratio of cross sections $d \sigma_{L} / d t$ for $\phi$ and $\rho$ production in model 1 . The meaning of the bands and solid lines is as in Fig. 22,
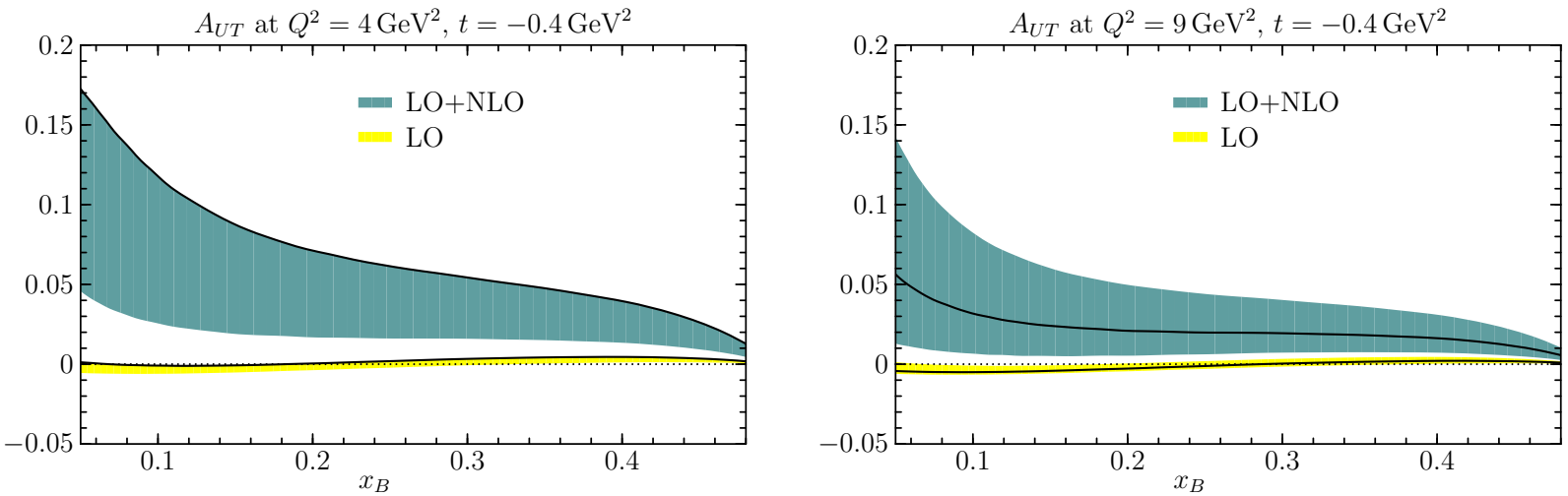

Figure 30: As Fig. 23 but for $\gamma^{*} p \rightarrow \phi p$. 


\section{Pseudoscalar meson production}

Having studied in detail the production of vector mesons, let us finally take a look at pseudoscalar production. We will only consider $\gamma^{*} p \rightarrow \pi^{+} n$, which was already studied at NLO in [10]. Gluon distributions do not contribute in this channel.

In the collinear approximation the amplitude for this process can be written as

$$
\begin{aligned}
\mathcal{M} & =\frac{4 \pi \sqrt{4 \pi \alpha}}{\xi Q N_{c}} f_{\pi} \int_{0}^{1} d z \phi_{\pi}(z) \int_{-1}^{1} d x\left[e_{u} T_{a}(\bar{z}, x, \xi)-e_{d} T_{a}(z,-x, \xi)\right]\left[\widetilde{F}^{u}(x, \xi, t)-\widetilde{F}^{d}(x, \xi, t)\right] \\
& =\frac{4 \pi \sqrt{4 \pi \alpha}}{\xi Q N_{c}} f_{\pi} \sum_{n=0}^{\infty} a_{n} \widetilde{\mathcal{F}}_{n}^{\pi}
\end{aligned}
$$

with $e_{u}=2 / 3, e_{d}=-1 / 3$ and $f_{\pi}=131 \mathrm{MeV} . \phi_{\pi}(z)$ is the twist-two distribution amplitude of the pion and has a Gegenbauer decomposition as in (15). The convolutions $\widetilde{\mathcal{F}}_{n}^{\pi}$ are defined as

$$
\widetilde{\mathcal{F}}_{n}^{\pi}=\int_{-1}^{1} d x\left[e_{u} T_{a, n}(x, \xi)-e_{d} T_{a}(-x, \xi)\right]\left[\widetilde{F}^{u}(x, \xi, t)-\widetilde{F}^{d}(x, \xi, t)\right],
$$

and the kernels $T_{a}(\bar{z}, x, \xi)$ and $T_{a, n}(x, \xi)$ are the same as in Sect. 2. The matrix elements $\widetilde{F}^{q}$ are the counterparts of $F^{q}$ for polarized quarks and given by

$$
\widetilde{F}^{q}(x, \xi, t)=\frac{1}{\left(p+p^{\prime}\right) \cdot n}\left[\widetilde{H}^{q}(x, \xi, t) \bar{u}\left(p^{\prime}\right) \not h \gamma_{5} u(p)+\widetilde{E}^{q}(x, \xi, t) \bar{u}\left(p^{\prime}\right) \frac{\left(p^{\prime}-p\right) \cdot n}{2 m_{p}} \gamma_{5} u(p)\right]
$$

in terms of the generalized parton distributions $\widetilde{H}$ and $\widetilde{E}$, where as in the unpolarized case we use the conventions of [17]. Since the hard-scattering kernel in (66) is neither even nor odd in $x$, the convolution involves both the charge-conjugation even and odd combinations

$$
\widetilde{F}^{q(+)}(x, \xi, t)=\widetilde{F}^{q}(x, \xi, t)+\widetilde{F}^{q}(-x, \xi, t), \quad \widetilde{F}^{q(-)}(x, \xi, t)=\widetilde{F}^{q}(x, \xi, t)-\widetilde{F}^{q}(-x, \xi, t) .
$$

We model the distributions $\widetilde{H}$ in close analogy to the unpolarized case and set

$$
\begin{aligned}
& \widetilde{H}^{q(+)}(x, \xi, t)=\int_{-1}^{1} d \beta \int_{-1+|\beta|}^{1-|\beta|} d \alpha \delta(x-\beta-\xi \alpha) h^{(2)}(\beta, \alpha) \widetilde{H}^{q(+)}(\beta, 0, t), \\
& \widetilde{H}^{q(-)}(x, \xi, t)=\int_{-1}^{1} d \beta \int_{-1+|\beta|}^{1-|\beta|} d \alpha \delta(x-\beta-\xi \alpha) h^{(2)}(\beta, \alpha) \widetilde{H}^{q(-)}(\beta, 0, t),
\end{aligned}
$$

with $h^{(2)}(\beta, \alpha)$ as in (24) and

$$
\begin{aligned}
\widetilde{H}^{q(+)}(x, 0, t) & =\Delta q_{v}(x) \exp \left[t f_{q_{v}}(x)\right]+2 \Delta \bar{q}(x) \exp \left[t f_{\bar{q}}(x)\right], \\
\widetilde{H}^{q(-)}(x, 0, t) & =\Delta q_{v}(x) \exp \left[t f_{q_{v}}(x)\right]
\end{aligned}
$$

for $x>0$. The values for $x<0$ are determined by the symmetry properties following from (68). For the polarized valence and antiquark densities $\Delta q_{v}$ and $\Delta \bar{q}$ we use the NLO parameterization from [41] at $\mu=2 \mathrm{GeV}$, and for the $t$ dependence we take the same functions $f_{q_{v}}(x)$ as in (26), (27) and furthermore set $f_{\bar{q}}(x)=f_{q_{v}}(x)$. As was shown in [28], this gives a good description of the isovector axial form factor via the sum rule

$$
F_{A}(t)=\int_{0}^{1} d x\left[\widetilde{H}^{u(+)}(x, 0, t)-\widetilde{H}^{d(+)}(x, 0, t)\right] .
$$



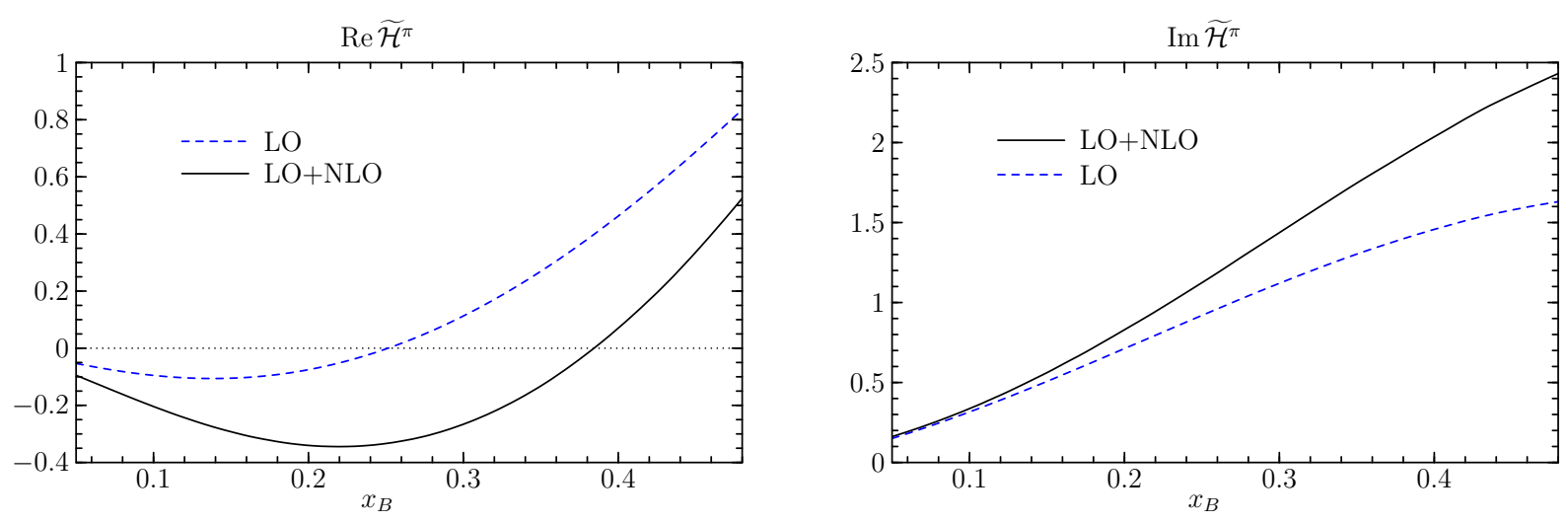

Figure 31: The convolution $\widetilde{\mathcal{H}}_{0}^{\pi}$ defined as in (66), evaluated at $Q=2 \mathrm{GeV}$ and $t=-0.4 \mathrm{GeV}^{2}$. The scales are set to $\mu_{R}=\mu_{G P D}=Q$.

For the nucleon helicity-flip distribution $\widetilde{E}$ we take a pion exchange ansatz

$$
\widetilde{E}^{u}(x, \xi, t)=-\widetilde{E}^{d}(x, \xi, t)=\frac{\theta(\xi-|x|)}{2 \xi} \phi_{\pi}\left(\frac{x+\xi}{2 \xi}\right) \frac{2 m_{p}^{2} g_{A}}{m_{\pi}^{2}-t} \frac{\Lambda^{2}-m_{\pi}^{2}}{\Lambda^{2}-t}
$$

with the nucleon axial charge $g_{A} \approx 1.26$ and a cutoff parameter $\Lambda=0.8 \mathrm{GeV}$ [42] to suppress large off-shellness of the exchanged pion in the $t$ channel.

\subsection{Results}

The convolution $\widetilde{\mathcal{H}}_{n}^{\pi}$ at LO and NLO is shown in Fig. 31 for $n=0$. We find moderate corrections for the imaginary part and larger ones for the real part. For $\widetilde{\mathcal{E}}_{n}^{\pi}$ we can easily give the analytic form of the NLO result. The scale dependent terms admit a closed expression,

$$
\begin{aligned}
\sum_{n} a_{n} \widetilde{\mathcal{E}}_{n}^{\pi} \propto \sum_{m, n} a_{m} a_{n}\left\{1+\frac{\alpha_{s}}{4 \pi}\right. & \\
\times & {\left.\left[\beta_{0}\left(\frac{14}{3}+\frac{\gamma_{m}+\gamma_{n}}{2 C_{F}}-\ln \frac{Q^{2}}{\mu_{R}^{2}}\right)-\gamma_{m} \ln \frac{Q^{2}}{\mu_{G P D}^{2}}-\gamma_{n} \ln \frac{Q^{2}}{\mu_{D A}^{2}}+\ldots\right]\right\}, }
\end{aligned}
$$

where the ... denote contributions which depend neither on $Q^{2}$ and the scales nor on $\beta_{0}$. Including these terms we can write

$$
\begin{aligned}
\sum_{n} a_{n} \widetilde{\mathcal{E}}_{n}^{\pi} \propto\left(1+a_{2}+a_{4}\right)^{2}+\frac{\alpha_{s}\left(\mu_{R}\right)}{\pi}\left[\frac{79}{12}+25.0 a_{2}+32.8 a_{4}+53.4 a_{2} a_{4}+21.4 a_{2}^{2}+32.6 a_{4}^{2}\right. \\
\left.-\frac{9}{4}\left(1+a_{2}+a_{4}\right)^{2} \ln \frac{Q^{2}}{\mu_{R}^{2}}-\left(1+a_{2}+a_{4}\right)\left(\frac{25}{18} a_{2}+\frac{91}{45} a_{4}\right)\left(\ln \frac{Q^{2}}{\mu_{G P D}^{2}}+\ln \frac{Q^{2}}{\mu_{D A}^{2}}\right)\right]+\ldots
\end{aligned}
$$

where we have set $n_{f}=3$ in $\beta_{0}$ and where we approximated numerically the coefficients written with a decimal point. Here the ... denote terms with higher Gegenbauer coefficients. Note that these coefficients appear twice, once for the produced pion and once for the pion exchange ansatz of the distribution $\widetilde{E}$. Up to a global factor, the expression (74) also gives the NLO result for the electromagnetic pion form factor $F_{\pi}\left(Q^{2}\right)$ at large spacelike momentum transfer $Q^{2}$, and it agrees with the result in the detailed study [9]. Let us first discuss the case $m=n=0$ relevant for the asymptotic 
form of the pion distribution amplitude, where the convolution has no dependence on $\mu_{G P D}$ and $\mu_{D A}$. We then have the rather large coefficient $79 / 12 \approx 6.6$ in square brackets, so that with the scale choice $\mu_{R}=Q$ there are quite large NLO corrections. The corrections are zero for $\mu_{R}^{2}=e^{-79 / 27} Q^{2} \approx 0.05 Q^{2}$, which is outside the perturbative region for most cases relevant in practice. The BLM scale for this case is yet smaller: with (73) we reproduce the well-known result $\mu_{R}^{2}=e^{-14 / 3} Q^{2} \approx 0.01 Q^{2}[10$ ]. The coefficient of $\alpha_{s} / \pi$ in (174) is then $-47 / 12 \approx-3.9$ and thus again rather large, but of course the scale $\mu_{R}$ is outside the perturbative region for all experimentally relevant kinematics. We finally see in (74) that for higher Gegenbauer moments the correction terms are larger than for $m=n=0$. The reason for this is the same which we discussed in Sect. 5 for the convolutions $\mathcal{H}$. In (73) we also see that the BLM scale becomes smaller for higher $m$ and $n$.

The observables for exclusive pion production at leading order in $1 / Q$ are the same as for vector meson production, and the ep cross section is given as in (60). The cross section for a longitudinal photon and the transverse target asymmetry are now respectively given by

$$
\frac{d \sigma_{L}}{d t}=\frac{\pi^{2}}{9} \frac{\alpha}{Q^{6}} \frac{\left(2-x_{B}\right)^{2}}{1-x_{B}}\left(2 f_{\pi}\right)^{2}\left[\left(1-\xi^{2}\right)\left|\widetilde{\mathcal{H}}_{\pi}\right|^{2}-\xi^{2} t /\left(4 m_{p}^{2}\right)\left|\widetilde{\mathcal{E}}_{\pi}\right|^{2}-2 \xi^{2} \operatorname{Re}\left(\widetilde{\mathcal{E}}_{\pi}^{*} \widetilde{\mathcal{H}}_{\pi}\right)\right]
$$

and

$$
A_{U T}=-\frac{\sqrt{t_{0}-t}}{m_{p}} \frac{\xi \sqrt{1-\xi^{2}} \operatorname{Im}\left(\widetilde{\mathcal{E}}_{\pi}^{*} \widetilde{\mathcal{H}}_{\pi}\right)}{\left(1-\xi^{2}\right)\left|\widetilde{\mathcal{H}}_{\pi}\right|^{2}-\xi^{2} t /\left(4 m_{p}^{2}\right)\left|\widetilde{\mathcal{E}}_{\pi}\right|^{2}-2 \xi^{2} \operatorname{Re}\left(\widetilde{\mathcal{E}}_{\pi}^{*} \widetilde{\mathcal{H}}_{\pi}\right)}
$$

with

$$
\widetilde{\mathcal{H}}_{\pi}=\sum_{n=0}^{\infty} a_{n} \widetilde{\mathcal{H}}_{n}^{\pi}, \quad \quad \widetilde{\mathcal{E}}_{\pi}=\sum_{n=0}^{\infty} a_{n} \widetilde{\mathcal{E}}_{n}^{\pi} .
$$

For numerical estimates we take the asymptotic pion distribution amplitude in the following, setting $a_{n}=0$ for $n \geq 2$. We note that the recent lattice study [22] obtained a rather moderate value $a_{2}\left(\mu_{0}\right)=0.201(114)$ at $\mu_{0}=2 \mathrm{GeV}$.

In Fig. 32 we show the separate contributions from the terms with $\left|\widetilde{\mathcal{H}}_{\pi}\right|^{2}$ and with $\left|\widetilde{\mathcal{E}}_{\pi}\right|^{2}$ in (75), as well as the full result. We see that at the value of $t$ chosen here, the contribution from $\left|\widetilde{\mathcal{H}}_{\pi}\right|^{2}$ is more important, mainly because of the suppression factor $\left(\Lambda^{2}-m_{\pi}^{2}\right) /\left(\Lambda^{2}-t\right)$ in our model (72) for $\widetilde{E}$. The square of this factor is 0.36 at $t=-0.4 \mathrm{GeV}^{2}$.

We compare the LO and NLO results for the cross section in Fig. 33 and find that the NLO corrections are quite large, even at $Q^{2}=9 \mathrm{GeV}^{2}$. In contrast, the corrections for the beam spin asymmetry are very small as seen in Fig. [34, in line with the findings reported in [10]. Note that with our model $\widetilde{\mathcal{E}}_{\pi}$ is purely real, so that at intermediate $x_{B}$ the large relative NLO corrections in $\operatorname{Re} \widetilde{\mathcal{H}}_{\pi}$ do not affect the numerator of $A_{U T}$ in (75). Approximating the asymmetry as

$$
A_{U T} \approx-\frac{\sqrt{t_{0}-t}}{m_{p}} \frac{\xi \operatorname{Im}\left(\widetilde{\mathcal{E}}_{\pi}^{*} \widetilde{\mathcal{H}}_{\pi}\right)}{\left|\widetilde{\mathcal{H}}_{\pi}\right|^{2}}=-\frac{\sqrt{t_{0}-t}}{m_{p}}\left|\frac{\xi \widetilde{\mathcal{E}}_{\pi}}{\widetilde{\mathcal{H}}_{\pi}}\right| \sin \delta_{\pi}
$$

with $\delta_{\pi}=\arg \left(\widetilde{\mathcal{H}}_{\pi} / \widetilde{\mathcal{E}}_{\pi}\right)$, we can understand why only small corrections are seen in this case: the relative phase $\delta_{\pi}$ is well different from zero, and the NLO corrections increase both $\left|\widetilde{\mathcal{H}}_{\pi}\right|$ and $\left|\widetilde{\mathcal{E}}_{\pi}\right|$. 


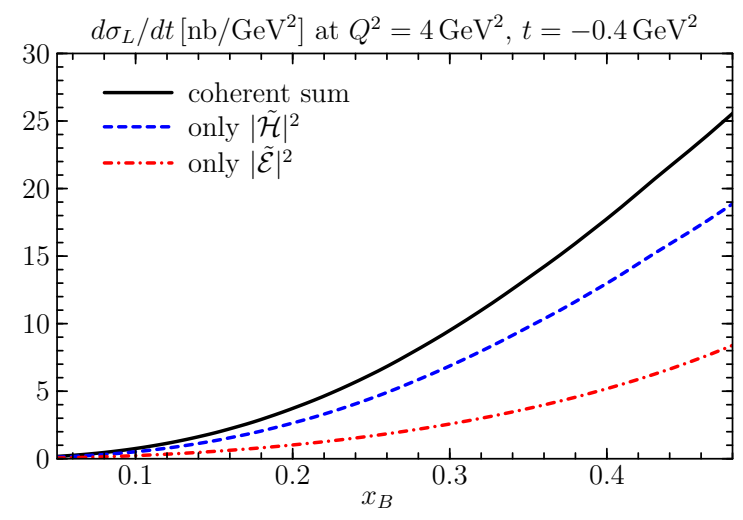

Figure 32: The longitudinal cross section for $\gamma^{*} p \rightarrow \pi^{+} n$, evaluated at NLO. Shown are the separate contributions from the terms with $\left|\widetilde{\mathcal{H}}_{\pi}\right|^{2}$ and with $\left|\widetilde{\mathcal{E}}_{\pi}\right|^{2}$ in $(\overline{75})$, as well as the complete expression.
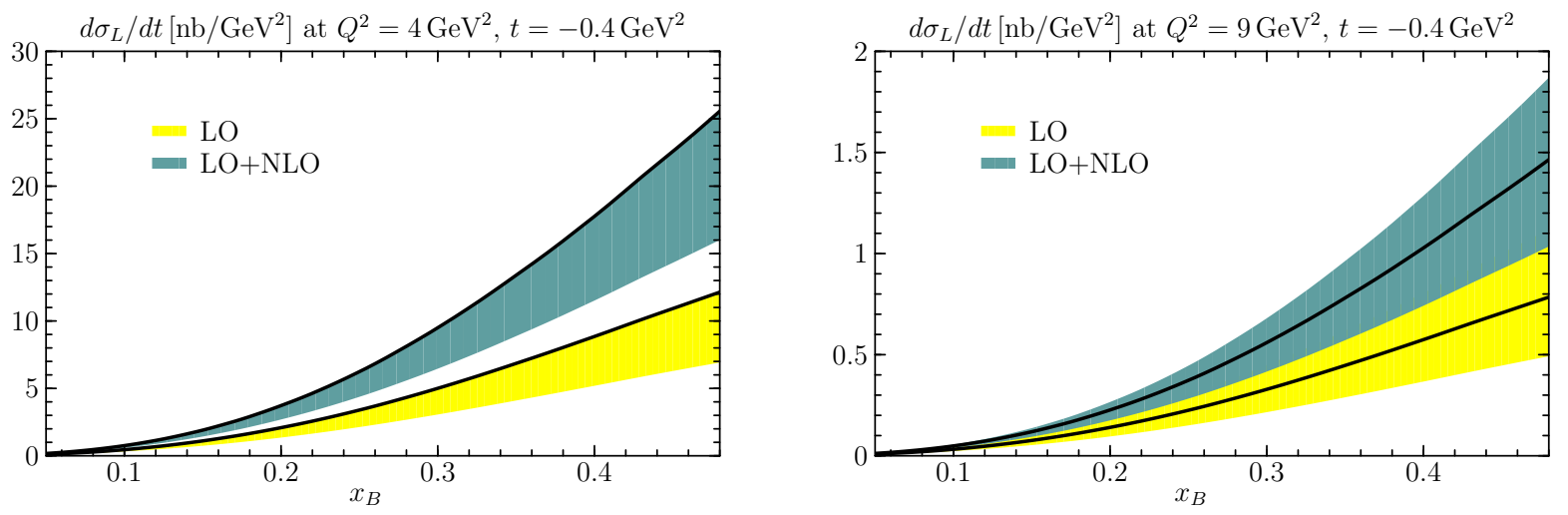

Figure 33: Longitudinal cross section for $\gamma^{*} p \rightarrow \pi^{+} n$. Bands correspond to the range $2 \mathrm{GeV}<\mu<$ $4 \mathrm{GeV}$ in the left and to $2 \mathrm{GeV}<\mu<6 \mathrm{GeV}$ in the right plot, and solid lines to $\mu=Q$ in both cases.
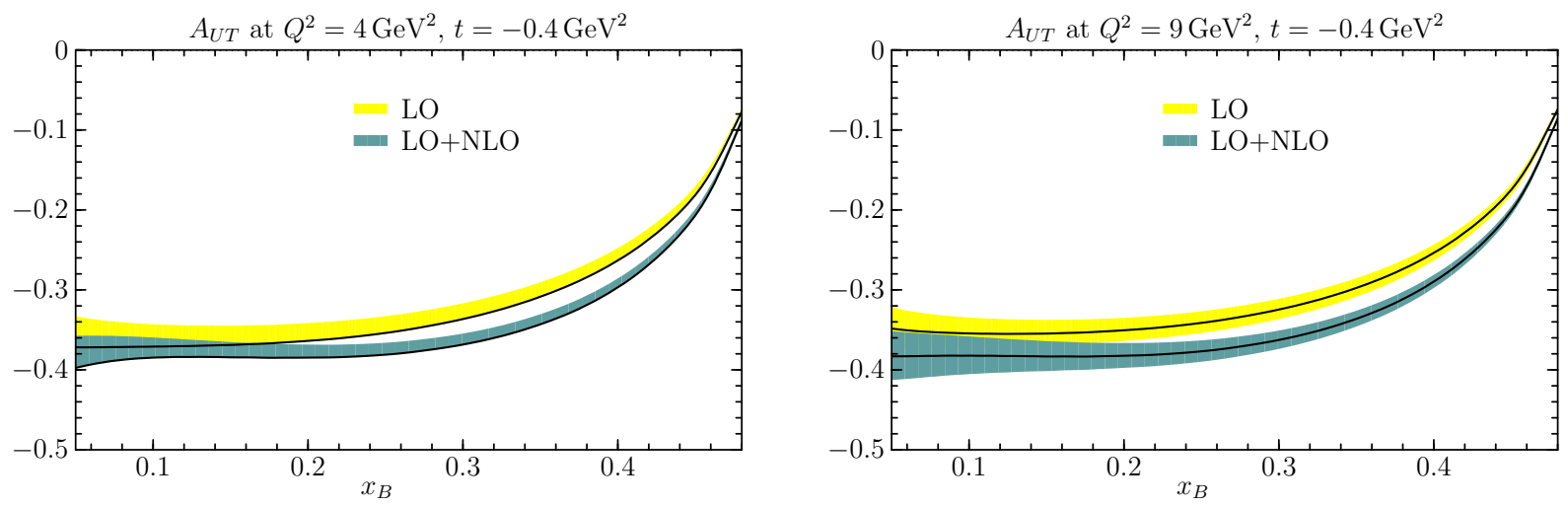

Figure 34: The transverse target spin asymmetry for $\pi^{+}$production, as defined in (60). The meaning of the bands and solid lines is as in Fig. 33 . 


\section{Summary}

In this work we have analyzed the NLO corrections for exclusive meson production at large $Q^{2}$ in the collinear factorization approach. Using the Gegenbauer expansion of meson distribution amplitudes, we have rewritten the hard-scattering kernels of [11] into functions depending on only one variable, and we have separated the explicit logarithms in the factorization scale for the meson distribution amplitude and the generalized parton distributions.

For vector meson production at small $x_{B}$ we find huge NLO corrections even for $Q^{2}$ well above $10 \mathrm{GeV}^{2}$, in agreement with the results obtained in [11. The corrections have opposite sign compared to the Born term and can be traced back to BFKL type logarithms in the hard-scattering kernels, which appear with rather large numerical prefactors in this process. We conclude at this stage that a quantitative control of radiative corrections at small $x_{B}$ will require resummation of these logarithms. First steps in this direction have been reported in [43. If successful, such a resummation in combination with a dispersion relation [39] may also be useful for stabilizing the real part of the amplitude, where we find very large NLO corrections even at $x_{B} \sim 0.1$.

At intermediate to large $x_{B}$, typical of fixed-target experiments, we have investigated the production of $\rho^{0}, \omega, \phi$ and of $\pi^{+}$. We find NLO corrections to the longitudinal cross sections of up to $100 \%$, which somewhat decrease in size when going from $Q^{2}=4 \mathrm{GeV}^{2}$ to $9 \mathrm{GeV}^{2}$. Note that the meson production cross section depends quadratically on generalized parton distributions - the increased sensitivity to these basic quantities comes with an increased sensitivity to higher-order corrections. We generally find that uncertainties on the cross section due to the choice of renormalization and factorization scales are not too large at LO and do not significantly decrease when going to NLO. For scales below $4 \mathrm{GeV}^{2}$, however, NLO corrections often grow out of control. The cross section ratio for $\omega$ to $\rho$ production turns out to be very stable under corrections, but less so the one for $\phi$ to $\rho$. For the transverse target polarization asymmetry $A_{U T}$ in $\pi^{+}$production we find quite small NLO effects, confirming the results in [10]. For vector meson production this is however not the case. With the models we have used for the nucleon helicity-flip distributions $E$, the numerator of the asymmetry in this channel is dominated by the product $\left(\operatorname{Im} \mathcal{E}_{V}\right)\left(\operatorname{Re} \mathcal{H}_{V}\right)$ in a wide range of kinematics and therefore suffers from the perturbative instability we find for $\operatorname{Re} \mathcal{H}_{V}$ at small to intermediate $x_{B}$, even if the corrections to $\operatorname{Im} \mathcal{E}_{V}$ are not too large. It is often assumed that corrections tend to cancel in asymmetries. The examples we have studied show that this may hold in specific cases but not in others, and that special care is needed for observables like $A_{U T}$ that depend on the relative phase between amplitudes.

We should recall that in the kinematics we studied, one must expect that our leading-twist results receive power corrections that cannot be neglected when comparing with data. They will certainly affect the cross sections and will not always cancel in cross section ratios. An example is the transverse target polarization asymmetry in $\pi^{+}$production. The phenomenological estimates in [12 found that the convolution $\widetilde{\mathcal{H}}_{\pi}$ is decreased by effects of transverse parton momentum in the hard scattering, whereas $\widetilde{\mathcal{E}}_{\pi}$ is increased by the soft overlap mechanism that has been extensively studied in the context of the pion form factor. Together, these corrections may significantly increase leading-twist estimates for $A_{U T}$.

From our numerical studies we must conclude that a precise quantitative interpretation of exclusive meson production requires large $Q^{2}$, say above $10 \mathrm{GeV}^{2}$. In addition it would be highly valuable to have a consistent scheme for combining radiative with power corrections, at least in parts. Nevertheless, we find that valuable information on generalized parton distributions can be obtained also from data at lower $Q^{2}$. In particular, a large measured asymmetry $A_{U T}$ in vector meson production would give valuable constraints on the size of the proton helicity-flip distribution $E^{g}$ for gluons, which are most difficult to obtain in deeply virtual Compton scattering or from lattice QCD calculations. 


\section{Acknowledgments}

We gratefully acknowledge discussions with L. Favart, H. Fischer, P. Kroll, A. Rostomyan and A. Schäfer. Special thanks are due to D. Yu. Ivanov for numerous conversations and advice. This work is supported by the Helmholtz Association, contract number VH-NG-004.

\section{A Polylogarithms}

We collect here some properties of the polylogarithms that appear in the hard-scattering kernels for meson production. Their definitions are

$$
\mathrm{Li}_{2} z=-\int_{0}^{1} \frac{d t}{t} \ln (1-z t), \quad \quad \operatorname{Li}_{3} z=\int_{0}^{1} \frac{d t}{t} \operatorname{Li}_{2}(z t),
$$

from which one readily obtains for the imaginary parts

$$
\operatorname{Im}\left[\operatorname{Li}_{2}(\bar{y}+i \epsilon)\right]=\pi \theta(-y) \ln \bar{y}, \quad \quad \operatorname{Im}\left[\operatorname{Li}_{3}(\bar{y}+i \epsilon)\right]=\frac{\pi}{2} \theta(-y) \ln ^{2} \bar{y} .
$$

The limiting behavior for $y \rightarrow-\infty$ can be obtained from the expansions

$$
\mathrm{Li}_{2} y=-\frac{\pi^{2}}{6}-\frac{1}{2} \ln ^{2}(-y)-\sum_{n=1}^{\infty} \frac{y^{-n}}{n^{2}}, \quad \operatorname{Li}_{3} y=-\frac{\pi^{2}}{6} \ln (-y)-\frac{1}{6} \ln ^{3}(-y)+\sum_{n=1}^{\infty} \frac{y^{-n}}{n^{3}},
$$

which are valid for $y<-1$, and from

$$
\operatorname{Re}\left[\operatorname{Li}_{2} \bar{y}\right]=\frac{\pi^{2}}{3}-\frac{1}{2} \ln ^{2} \bar{y}-\sum_{n=1}^{\infty} \frac{\bar{y}^{-n}}{n^{2}}, \quad \operatorname{Re}\left[\operatorname{Li}_{3} \bar{y}\right]=\frac{\pi^{2}}{3} \ln \bar{y}-\frac{1}{6} \ln ^{3} \bar{y}+\sum_{n=1}^{\infty} \frac{\bar{y}^{-n}}{n^{3}},
$$

which holds for $y<0$. A useful relation finally is

$$
\operatorname{Li}_{2} y+\operatorname{Li}_{2} \bar{y}=\frac{\pi^{2}}{6}-(\ln y)(\ln \bar{y}) .
$$

A wealth of further information can be found in [44].

\section{B Hard-scattering kernels for higher Gegenbauer moments}

In this appendix we give the analogs of the hard-scattering kernels in (21) for Gegenbauer index $n=2$ and $n=4$. For the gluon kernel we find

$$
\begin{aligned}
t_{g, 2}(y)= & {\left[2 C_{A}\left(y^{2}+\bar{y}^{2}\right)-C_{F} y\right] \frac{\ln y}{\bar{y}} \ln \frac{Q^{2}}{\mu_{G P D}^{2}}+\frac{\beta_{0}}{2} \ln \frac{\mu_{R}^{2}}{\mu_{G P D}^{2}}-\frac{25}{12} C_{F} \ln \frac{Q^{2}}{\mu_{D A}^{2}} } \\
+ & C_{F}\left[\frac{35}{36}(5-54 y \bar{y})-\frac{y}{2} \frac{\ln ^{2} y}{\bar{y}}-7(\bar{y}-y)(1-30 y \bar{y}) \operatorname{Li}_{2} \bar{y}\right. \\
& \left.+\left(\frac{1}{\bar{y}}-\frac{3}{2}-\frac{392}{3} y+525 y^{2}-420 y^{3}\right) \ln y\right] \\
+ & C_{A}\left[-\frac{15}{4}(1-4 y \bar{y})+\left(\frac{1}{\bar{y}}-2 y\right) \ln ^{2} y+(\bar{y}-y)(7-60 y \bar{y}) \operatorname{Li}_{2} \bar{y}\right. \\
& \left.-\left(\frac{23}{3 \bar{y}}+\frac{5}{6}-58 y+150 y^{2}-120 y^{3}\right) \ln y\right]
\end{aligned}
$$




$$
\begin{aligned}
&+ 6 y \bar{y}\left[5(1-4 y \bar{y}) C_{A}-14(1-5 y \bar{y}) C_{F}\right]\left(3 \operatorname{Li}_{3} \bar{y}-\ln y \operatorname{Li}_{2} y-\frac{\pi^{2}}{6} \ln y\right)+\{y \rightarrow \bar{y}\}, \\
& t_{g, 4}(y)=\left[2 C_{A}\left(y^{2}+\bar{y}^{2}\right)-C_{F} y\right] \frac{\ln y}{\bar{y}} \ln \frac{Q^{2}}{\mu_{G P D}^{2}}+\frac{\beta_{0}}{2} \ln \frac{\mu_{R}^{2}}{\mu_{G P D}^{2}}-\frac{91}{30} C_{F} \ln \frac{Q^{2}}{\mu_{D A}^{2}} \\
&+C_{F}\left[\frac{27287}{1800}-595 y \bar{y}+2520(y \bar{y})^{2}-\frac{y}{2} \frac{\ln ^{2} y}{\bar{y}}+16(\bar{y}-y)\left(1-105 y \bar{y}+630(y \bar{y})^{2}\right) \mathrm{Li}_{2} \bar{y}\right. \\
&\left.+\left(\frac{1}{\bar{y}}-\frac{5}{2}-\frac{11596}{15} y+9660 y^{2}-34160 y^{3}+45360 y^{4}-20160 y^{5}\right) \ln y\right] \\
&+C_{A}[-\frac{35}{16}(1-4 y \bar{y})(5-72 y \bar{y})+\left(\frac{1}{\bar{y}}-2 y\right) \ln ^{2} y+2(\bar{y}-y)\left(8-315 y \bar{y}+1260(y \bar{y})^{2}\right) \operatorname{Li}_{2} \bar{y} \\
&\left.-\left(\frac{257}{30 \bar{y}}+\frac{77}{60}-\frac{1741}{5} y+2940 y^{2}-8960 y^{3}+11340 y^{4}-5040 y^{5}\right) \ln y\right] \\
&+30 y \bar{y} {\left[7(1-4 y \bar{y})(1-6 y \bar{y}) C_{A}\right.} \\
&\left.-16\left(1-14 y \bar{y}+42(y \bar{y})^{2}\right) C_{F}\right]\left(3 \operatorname{Li}_{3} \bar{y}-\ln y \operatorname{Li}_{2} y-\frac{\pi^{2}}{6} \ln y\right)+\{y \rightarrow \bar{y}\}
\end{aligned}
$$

and for the pure singlet kernel

$$
\begin{aligned}
t_{b, 2}(y)= & 2(\bar{y}-y) \frac{\ln y}{\bar{y}}\left[\ln \frac{Q^{2}}{\mu_{G P D}^{2}}-\frac{23}{6}\right]+(\bar{y}-y) \frac{\ln ^{2} y}{\bar{y}}-\frac{15}{2}(\bar{y}-y) \\
& +2(7-60 y \bar{y}) \operatorname{Li}_{2} \bar{y}-\left(\frac{5}{3}-90 y+120 y^{2}\right) \ln y \\
& +60(\bar{y}-y) y \bar{y}\left[3 \operatorname{Li}_{3} \bar{y}+\left(\operatorname{Li}_{2} \bar{y}+\ln ^{2} \bar{y}-\frac{\pi^{2}}{3}\right) \ln y\right]-\{y \rightarrow \bar{y}\}, \\
t_{b, 4}(y)= & 2(\bar{y}-y) \frac{\ln y}{\bar{y}}\left[\ln \frac{Q^{2}}{\mu_{G P D}^{2}}-\frac{257}{60}\right]+(\bar{y}-y) \frac{\ln ^{2} y}{\bar{y}}-\frac{35}{8}(\bar{y}-y)(5-72 y \bar{y}) \\
& +4\left(8-315 y \bar{y}+1260(y \bar{y})^{2}\right) \operatorname{Li}_{2} \bar{y}-\left(\frac{77}{30}-665 y+4550 y^{2}-8820 y^{3}+5040 y^{4}\right) \ln y \\
& +420(\bar{y}-y) y \bar{y}(1-6 y \bar{y})\left[3 \operatorname{Li}_{3} \bar{y}+\left(\operatorname{Li}_{2} \bar{y}+\ln ^{2} \bar{y}-\frac{\pi^{2}}{3}\right) \ln y\right]-\{y \rightarrow \bar{y}\} .
\end{aligned}
$$

The quark non-singlet kernel reads

$$
\begin{aligned}
t_{a, 2}(y)= & \beta_{0}\left[\frac{21}{4}-\ln y-\ln \frac{Q^{2}}{\mu_{R}^{2}}\right] \\
+ & C_{F}\left[(3+2 \ln y) \ln \frac{Q^{2}}{\mu_{G P D}^{2}}-\frac{25}{6} \ln \frac{Q^{2}}{\mu_{D A}^{2}}-\frac{1019}{72}-\left(\frac{1}{\bar{y}}+\frac{7}{6}\right) \ln y+\ln ^{2} y\right] \\
+ & \left(2 C_{F}-C_{A}\right)\left\{\frac{401}{12}-255 y+270 y^{2}-\left(\frac{299}{3}-867 y+1830 y^{2}-1080 y^{3}\right) \ln \bar{y}\right. \\
& +\left(\frac{56}{3}-357 y+1290 y^{2}-1080 y^{3}\right) \ln y+2\left(22-291 y+780 y^{2}-540 y^{3}\right)\left(\operatorname{Li}_{2} y-\operatorname{Li}_{2} \bar{y}\right) \\
& +12\left(1-21 y+106 y^{2}-175 y^{3}+90 y^{4}\right) \\
& \left.\times\left[3\left(\operatorname{Li}_{3} \bar{y}+\operatorname{Li}_{3} y\right)-\ln y \operatorname{Li}_{2} y-\ln \bar{y} \operatorname{Li}_{2} \bar{y}-\frac{\pi^{2}}{6}(\ln y+\ln \bar{y})\right]\right\},
\end{aligned}
$$




$$
\begin{aligned}
t_{a, 4}(y)= & \beta_{0}\left[\frac{31}{5}-\ln y-\ln \frac{Q^{2}}{\mu_{R}^{2}}\right] \\
+ & C_{F}\left[(3+2 \ln y) \ln \frac{Q^{2}}{\mu_{G P D}^{2}}-\frac{91}{15} \ln \frac{Q^{2}}{\mu_{D A}^{2}}-\frac{10213}{900}-\left(\frac{1}{\bar{y}}+\frac{46}{15}\right) \ln y+\ln ^{2} y\right] \\
+ & \left(2 C_{F}-C_{A}\right)\left\{\frac{4903}{40}-\frac{5775}{2} y+\frac{57085}{4} y^{2}-23310 y^{3}+11970 y^{4}\right. \\
& -\left(\frac{21109}{60}-\frac{41451}{5} y+\frac{103285}{2} y^{2}-125020 y^{3}+129150 y^{4}-47880 y^{5}\right) \ln \bar{y} \\
+ & \left(\frac{2899}{60}-\frac{11001}{5} y+\frac{45535}{2} y^{2}-78400 y^{3}+105210 y^{4}-47880 y^{5}\right) \ln y \\
+ & \left(137-4506 y+35280 y^{2}-100380 y^{3}+117180 y^{4}-47880 y^{5}\right)\left(\operatorname{Li}_{2} y-\operatorname{Li}_{2} \bar{y}\right) \\
+ & 30\left(1-48 y+580 y^{2}-2590 y^{3}+5166 y^{4}-4704 y^{5}+1596 y^{6}\right) \\
& \left.\times\left[3\left(\operatorname{Li}_{3} \bar{y}+\operatorname{Li}_{3} y\right)-\ln y \operatorname{Li}_{2} y-\ln \bar{y} \operatorname{Li}_{2} \bar{y}-\frac{\pi^{2}}{6}(\ln y+\ln \bar{y})\right]\right\}
\end{aligned}
$$

Using (11), (12) and the representation

$$
\gamma_{n}=(-1)^{n+1} 2 C_{F} \int_{0}^{1} d z(1-z)(3+2 \ln z) C_{n}^{3 / 2}(2 z-1)
$$

of the anomalous dimensions, we can give a closed form for the scale dependent terms for all even $n$,

$$
\begin{aligned}
& t_{g, n}(y)=\left[2 C_{A}\left(y^{2}+\bar{y}^{2}\right)-C_{F} y\right] \frac{\ln y}{\bar{y}} \ln \frac{Q^{2}}{\mu_{G P D}^{2}}+\frac{\beta_{0}}{2} \ln \frac{\mu_{R}^{2}}{\mu_{G P D}^{2}}-\frac{\gamma_{n}}{2} \ln \frac{Q^{2}}{\mu_{D A}^{2}}+\{y \rightarrow \bar{y}\}+\ldots, \\
& t_{b, n}(y)=2(\bar{y}-y) \frac{\ln y}{\bar{y}} \ln \frac{Q^{2}}{\mu_{G P D}^{2}}-\{y \rightarrow \bar{y}\}+\ldots, \\
& t_{a, n}(y)=\beta_{0}\left[\frac{19}{6}+\frac{\gamma_{n}}{2 C_{F}}-\ln y-\ln \frac{Q^{2}}{\mu_{R}^{2}}\right]+C_{F}(3+2 \ln y) \ln \frac{Q^{2}}{\mu_{G P D}^{2}}-\gamma_{n} \ln \frac{Q^{2}}{\mu_{D A}^{2}}+\ldots,
\end{aligned}
$$

where the terms denoted by ... are independent of $Q^{2}$ and the scales and do not involve $\beta_{0}$. From the scale dependence (16) of the Gegenbauer coefficients of the meson distribution amplitude we can readily reconstruct their evolution equation

$$
\mu^{2} \frac{\mathrm{d}}{\mathrm{d} \mu^{2}} a_{n}(\mu)=-\frac{\alpha_{s}(\mu)}{4 \pi} \gamma_{n} a_{n}(\mu)+O\left(\alpha_{s}^{2}\right)
$$

With (20) and (88) we see that the $\mu_{D A}$ dependence of the process amplitude (18) cancels up to terms of order $\alpha_{s}^{3}$, as it must be.

\section{Evolution kernels}

For definiteness we give here the LO evolution kernels for GPDs, which we have used to check the scale invariance of the NLO amplitude for meson production as explained in Sect. 2. The non-singlet evolution equation reads

$$
\mu^{2} \frac{\mathrm{d}}{\mathrm{d} \mu^{2}} F^{N S}(x, \xi, t)=\int_{-1}^{1} \frac{d y}{|\xi|} V^{N S}\left(\frac{x}{\xi}, \frac{y}{\xi}\right) F^{N S}(y, \xi, t),
$$


where $F^{N S}$ can be a flavor non-singlet combination such as $F^{u(+)}-F^{d(+)}$, or the charge-conjugation odd combination $F^{q(-)}(x, \xi, t)=F^{q}(x, \xi, t)+F^{q}(-x, \xi, t)$ for a single quark flavor. In the gluon and quark singlet sector we have a matrix equation

$$
\mu^{2} \frac{\mathrm{d}}{\mathrm{d} \mu^{2}}\left(\begin{array}{c}
F^{S}(x, \xi, t) \\
F^{g}(x, \xi, t)
\end{array}\right)=\int_{-1}^{1} \frac{d y}{|\xi|}\left(\begin{array}{cc}
V^{q q}\left(\frac{x}{\xi}, \frac{y}{\xi}\right) & \xi^{-1} V^{q g}\left(\frac{x}{\xi}, \frac{y}{\xi}\right) \\
\xi V^{g q}\left(\frac{x}{\xi}, \frac{y}{\xi}\right) & V^{g g}\left(\frac{x}{\xi}, \frac{y}{\xi}\right)
\end{array}\right)\left(\begin{array}{c}
F^{S}(y, \xi, t) \\
F^{g}(y, \xi, t)
\end{array}\right)
$$

with $F^{S}$ defined in (5). At $O\left(\alpha_{s}\right)$ one has $V^{N S}(x, y)=V^{q q}(x, y)$ and

$$
\begin{aligned}
V^{q q}(x, y) & =\frac{\alpha_{s}}{4 \pi} C_{F}\left[\rho(x, y) \frac{1+x}{1+y}\left(1+\frac{2}{y-x}\right)+\{x \rightarrow-x, y \rightarrow-y\}\right]_{+}, \\
V^{q g}(x, y) & =-\frac{\alpha_{s}}{4 \pi} 2 T_{F} n_{f}\left[\rho(x, y) \frac{1+x}{(1+y)^{2}}(1-2 x+y-x y)-\{x \rightarrow-x, y \rightarrow-y\}\right], \\
V^{g q}(x, y) & =\frac{\alpha_{s}}{4 \pi} C_{F}\left[\rho(x, y)\left((2-x)(1+x)^{2}-\frac{(1+x)^{2}}{1+y}\right)-\{x \rightarrow-x, y \rightarrow-y\}\right], \\
V^{g g}(x, y) & =\frac{\alpha_{s}}{4 \pi} C_{A}\left[\rho(x, y) \frac{(1+x)^{2}}{(1+y)^{2}}\left(2+\frac{2}{y-x}\right)+\{x \rightarrow-x, y \rightarrow-y\}\right]_{+} \\
& +\frac{\alpha_{s}}{4 \pi} C_{A}\left[\rho(x, y) \frac{(1+x)^{2}}{(1+y)^{2}}(1-2 x+2 y-x y)+\{x \rightarrow-x, y \rightarrow-y\}\right] \\
& +\frac{\alpha_{s}}{4 \pi}\left(\beta_{0}-\frac{14}{3} C_{A}\right) \delta(x-y)
\end{aligned}
$$

with $T_{F}=1 / 2$ and the remaining constants as given in (13). The plus-prescription appearing in $V^{q q}$ and $V^{g g}$ is defined by

$$
[f(x, y)]_{+}=f(x, y)-\delta(x-y) \int d z f(z, y)
$$

and the function $\rho(x, y)$ specifies the support as

$$
\rho(x, y)=\theta\left(\frac{1+x}{1+y}\right) \theta\left(1-\frac{1+x}{1+y}\right) \operatorname{sgn}(1+y)=\theta(y-x) \theta(x+1)-\theta(x-y) \theta(-x-1) .
$$

The evolution equations for polarized GPDs read as in (90) and (91), with the unpolarized matrix elements $F$ and kernels $V$ replaced by their polarized counterparts $\widetilde{F}$ and $\widetilde{V}$. With $\widetilde{F}^{q(+)}$ and $\widetilde{F}^{q(-)}$ defined in (68) above, $\widetilde{F}^{N S}$ can be either a flavor non-singlet combination like $\widetilde{F}^{u(+)}-\widetilde{F}^{d(+)}$ or a charge-conjugation odd combination $\widetilde{F}^{q(-)}$, whereas the flavor singlet combination is given by

$$
\widetilde{F}^{S}=\widetilde{F}^{u(+)}+\widetilde{F}^{d(+)}+\widetilde{F}^{s(+)} .
$$

To $O\left(\alpha_{s}\right)$ the polarized evolution kernels are

$$
\widetilde{V}^{N S}(x, y)=\widetilde{V}^{q q}(x, y)=V^{q q}(x, y)
$$

and

$$
\begin{aligned}
& \widetilde{V}^{q g}(x, y)=-\frac{\alpha_{s}}{4 \pi} 2 T_{f} n_{f}\left[\rho(x, y) \frac{1+x}{(1+y)^{2}}-\{x \rightarrow-x, y \rightarrow-y\}\right], \\
& \widetilde{V}^{g q}(x, y)=\frac{\alpha_{s}}{4 \pi} C_{F}\left[\rho(x, y) \frac{(1+x)^{2}}{1+y}-\{x \rightarrow-x, y \rightarrow-y\}\right]
\end{aligned}
$$




$$
\begin{aligned}
\widetilde{V}^{g g}(x, y) & =\frac{\alpha_{s}}{4 \pi} C_{A}\left[\rho(x, y) \frac{(1+x)^{2}}{(1+y)^{2}}\left(2+\frac{2}{y-x}\right)+\{x \rightarrow-x, y \rightarrow-y\}\right]_{+} \\
& +\frac{\alpha_{s}}{4 \pi}\left(\beta_{0}-\frac{14}{3} C_{A}\right) \delta(x-y) .
\end{aligned}
$$

The kernels given here agree with those in [45] if one takes into account that any contribution to $V^{g q}(x, y)$ which is even in $y$ at fixed $x$ will drop out in the convolution (91). Taking the limit $\xi \rightarrow 0$ as

$$
\lim _{\xi \rightarrow 0^{+}} \frac{1}{\xi}\left(\begin{array}{cc}
V^{q q}\left(\frac{z}{\xi}, \frac{1}{\xi}\right) & \frac{1}{\xi} V^{q g}\left(\frac{z}{\xi}, \frac{1}{\xi}\right) \\
\frac{\xi}{z} V^{g q}\left(\frac{z}{\xi}, \frac{1}{\xi}\right) & \frac{1}{z} V^{g g}\left(\frac{z}{\xi}, \frac{1}{\xi}\right)
\end{array}\right)=\left(\begin{array}{cc}
P^{q q}(z) & P^{q g}(z) \\
P^{g q}(z) & P^{g g}(z)
\end{array}\right)
$$

one obtains the usual DGLAP evolution kernels from (92), and in analogy one recovers the polarized

DGLAP kernels from (97). The factors $\frac{1}{z}$ in front of $V^{g q}$ and $V^{g g}$ reflect the different forward limits of the quark and gluon GPDs.

\section{References}

[1] M. Burkardt, Int. J. Mod. Phys. A 18 (2003) 173 hep-ph/0207047.

[2] X. D. Ji, Phys. Rev. Lett. 78 (1997) 610 hep-ph/9603249].

[3] M. Burkardt and G. Schnell, Phys. Rev. D 74 (2006) 013002 hep-ph/0510249].

[4] A. V. Belitsky, D. Müller and A. Kirchner, Nucl. Phys. B629 (2002) 323 hep-ph/0112108.

[5] K. Kumerički, D. Müller and K. Passek-Kumerički, hep-ph/0703179.

[6] A. Levy [ZEUS Collaboration], arXiv:0706.1867 [hep-ex];

X. Janssen [H1 Collaboration], Acta Phys. Polon. B 33 (2002) 3529 hep-ex/0207011.

[7] C. Hadjidakis, D. Hasch and E. Thomas [HERMES Collaboration], Int. J. Mod. Phys. A 20 (2005) 593 [hep-ex/0405078];

C. Hadjidakis et al. [CLAS Collaboration], Phys. Lett. B 605 (2005) 256 [hep-ex/0408005];

A. Sandacz [COMPASS Collaboration], AIP Conf. Proc. 842 (2006) 345;

A. Rostomyan and J. Dreschler [HERMES Collaboration], arXiv:0707.2486 [hep-ex].

[8] J. C. Collins, L. Frankfurt and M. Strikman, Phys. Rev. D 56 (1997) 2982 hep-ph/9611433.

[9] B. Melić, B. Nižić and K. Passek, Phys. Rev. D 60 (1999) 074004 hep-ph/9802204.

[10] A. V. Belitsky and D. Müller, Phys. Lett. B 513 (2001) 349 [hep-ph/0105046].

[11] D. Yu. Ivanov, L. Szymanowski and G. Krasnikov, JETP Lett. 80 (2004) 226 hep-ph/0407207.

[12] M. Vanderhaeghen, P. A. M. Guichon and M. Guidal, Phys. Rev. D 60 (1999) 094017 hep-ph/9905372.

[13] S. V. Goloskokov and P. Kroll, Eur. Phys. J. C 50 (2007) 829 hep-ph/0611290.

[14] J. Botts and G. Sterman, Nucl. Phys. B 325 (1989) 62;

H.-n. Li and G. Sterman, Nucl. Phys. B 381 (1992) 129.

[15] L. Frankfurt, W. Koepf and M. Strikman, Phys. Rev. D 54 (1996) 3194 hep-ph/9509311. 
[16] A. D. Martin, M. G. Ryskin and T. Teubner, Phys. Rev. D 55 (1997) 4329 hep-ph/9609448.

[17] M. Diehl, Phys. Rept. 388 (2003) 41 hep-ph/0307382.

[18] M. Beneke and M. Neubert, Nucl. Phys. B 675 (2003) 333 hep-ph/0308039.

[19] A. V. Efremov and A. V. Radyushkin, Phys. Lett. B94 (1980) 245;

G. P. Lepage and S. J. Brodsky, Phys. Lett. B87 (1979) 359.

[20] P. Ball and V. M. Braun, Phys. Rev. D 54 (1996) 2182 hep-ph/9602323.

[21] A. P. Bakulev, S. V. Mikhailov and N. G. Stefanis, Phys. Rev. D 73 (2006) 056002 hep-ph/0512119.

[22] V. M. Braun et al. [QCDSF/UKQCD Collaboration], Phys. Rev. D 74 (2006) 074501 hep-lat/0606012.

[23] S. J. Brodsky, G. P. Lepage and P. B. Mackenzie, Phys. Rev. D 28 (1983) 228.

[24] I. V. Anikin et al., Eur. Phys. J. C 42 (2005) 163 hep-ph/0411408;

S. J. Brodsky and F. J. Llanes-Estrada, Eur. Phys. J. C 46 (2006) 751 hep-ph/0512247].

[25] A. P. Bakulev, A. V. Radyushkin and N. G. Stefanis, Phys. Rev. D 62 (2000) 113001 hep-ph/0005085.

[26] I. V. Musatov and A. V. Radyushkin, Phys. Rev. D 61 (2000) 074027 hep-ph/9905376.

[27] K. Goeke, M. V. Polyakov and M. Vanderhaeghen, Prog. Part. Nucl. Phys. 47 (2001) 401 hep-ph/0106012.

[28] M. Diehl, T. Feldmann, R. Jakob and P. Kroll, Eur. Phys. J. C 39 (2005) 1 hep-ph/0408173.

[29] J. Pumplin, D. R. Stump, J. Huston, H. L. Lai, P. Nadolsky and W. K. Tung, JHEP 0207 (2002) 012 hep-ph/0201195.

[30] A. Aktas et al. [H1 Collaboration], Eur. Phys. J. C 46 (2006) 585 hep-ex/0510016.

[31] M. Burkardt, Phys. Lett. B 582 (2004) 151 hep-ph/0309116.

[32] Ph. Hägler et al. [LHPC Collaboration], arXiv:0705.4295 [hep-lat].

[33] G. Schierholz [QCDSF Collaboration], Talk at the Workshop on Exclusive Reactions at High Momentum Transfer, Jefferson Lab, USA, 21-24 May 2007.

[34] A. V. Vinnikov, hep-ph/0604248.

[35] A. V. Belitsky, A. Freund and D. Müller, Nucl. Phys. B 574 (2000) 347 hep-ph/9912379.

[36] ZEUS Collaboration, paper 594 submitted to EPS 2001, Budapest, Hungary, 2001 http://www-zeus.desy.de/physics/phch/conf/eps01_paper.html

[37] D. Yu. Ivanov, A. Schäfer, L. Szymanowski and G. Krasnikov, Eur. Phys. J. C 34 (2004) 297 hep-ph/0401131.

[38] M. G. Ryskin, R. G. Roberts, A. D. Martin and E. M. Levin, Z. Phys. C 76 (1997) 231 hep-ph/9511228. 
[39] I. V. Anikin and O. V. Teryaev, arXiv:0704.2185 [hep-ph];

M. Diehl and D. Yu. Ivanov, arXiv:0707.0351 [hep-ph].

[40] A. Bacchetta, U. D'Alesio, M. Diehl and C. A. Miller, Phys. Rev. D 70 (2004) 117504 hep-ph/0410050].

[41] J. Blümlein and H. Böttcher, Nucl. Phys. B 636 (2002) 225 hep-ph/0203155].

[42] W. Koepf, L. L. Frankfurt and M. Strikman, Phys. Rev. D 53 (1996) 2586 hep-ph/9507218.

[43] D. Yu. Ivanov, Talk at the 12th International Conference on Elastic and Diffractive Scattering, Hamburg, Germany, 2007, to appear in the proceedings.

[44] A. Devoto and D. W. Duke, Riv. Nuovo Cim. 7N6 (1984) 1.

[45] J. Blümlein, B. Geyer and D. Robaschik, Nucl. Phys. B 560 (1999) 283 hep-ph/9903520. 